\title{
Deducing a variational principle with minimal a priori assumptions
}

\author{
Andrew Krieger* Georg Menz \\ Department of Mathematics \\ University of California \\ Los Angeles, U.S.A. \\ \{akrieger,gmenz\}@math.ucla.edu
}

\author{
Martin Tassy \\ Department of Mathematics \\ Dartmouth College \\ Hanover, U.S.A. \\ mtassy@math.dartmouth.edu
}

Submitted: Nov 6, 2019; Accepted: Aug 15, 2020; Published: Oct 2, 2020

(C) The authors. Released under the CC BY license (International 4.0).

\begin{abstract}
We study the well-known variational and large deviation principle for graph homomorphisms from $\mathbb{Z}^{m}$ to $\mathbb{Z}$. We provide a robust method to deduce those principles under minimal a priori assumptions. The only ingredient specific to the model is a discrete Kirszbraun theorem i.e. an extension theorem for graph homomorphisms. All other ingredients are of a general nature not specific to the model. They include elementary combinatorics, the compactness of Lipschitz functions, and a simplicial Rademacher theorem. Compared to the literature, our proof does not need any other preliminary results like e.g. concentration or strict convexity of the local surface tension. Therefore, the method is very robust and extends to more complex and subtle models, as e.g. the homogenization of limit shapes or graph-homomorphisms to a regular tree.
\end{abstract}

Mathematics Subject Classifications: 82B41, 82B20, 60F10

\section{Introduction}

Recently the study of limit shapes has attracted a lot of research. Limit shapes appear in many models, including domino tilings and dimer models (e.g. [Kas63, CEP96, CKP01]; see Figures 1a, 1b, and 1c), polymer models, lozenge tilings (e.g. [Des98, LRS01, Wil04]), Ginzburg-Landau models (e.g. [DGI00, FO04]), Gibbs models (e.g. [She05]), the Ising model (e.g. [DKS92, Cer06]), asymmetric exclusion processes (e.g. [FS06]), random matrices (e.g. [Wig59, KS99]), sandpile models (e.g.[LP08]), the six vertex model (e.g. [BCG16, CS16, RS18]), and Young tableaux (e.g. [LS77, VK77, PR07]). The appearance of limit shapes seems to be a universal phenomenon: it has been rigorously proven for many models, and it is strongly suggested by simulations for many additional models.

*Partially supported by NSF grant DMS-1712632. 


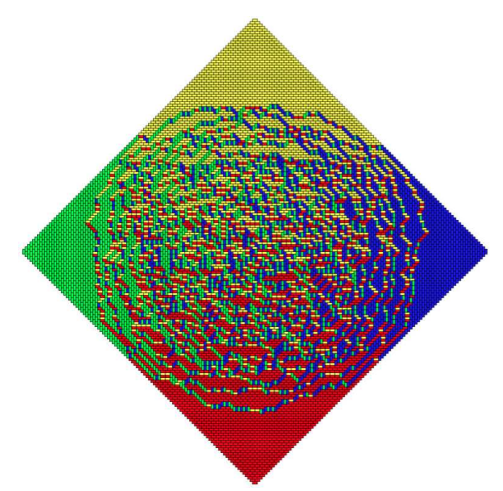

(a) Domino tiling (see [JPS98, CKP01]).

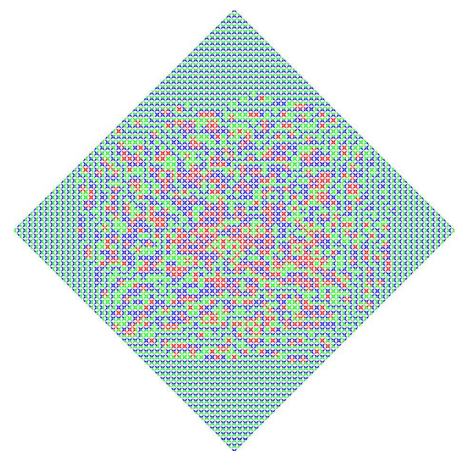

(d) Graph homomorphisms into the 3-regular tree (see [MT20]).

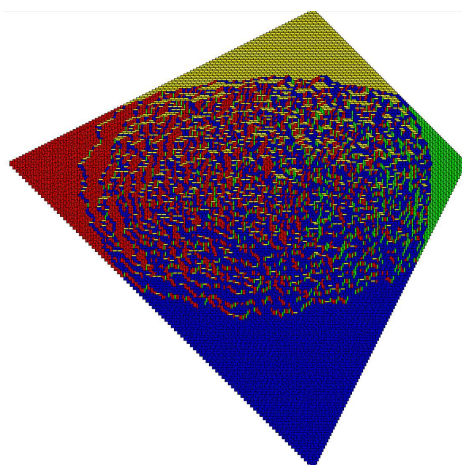

(b) Ribbon tiling (see [She01]).

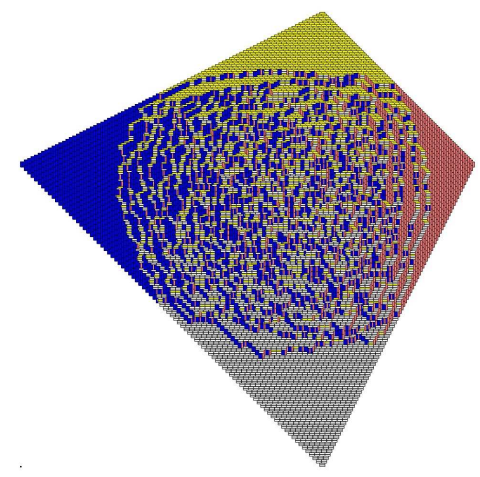

(c) Tiling by $3 \times 1$ bars (see [KK92]).

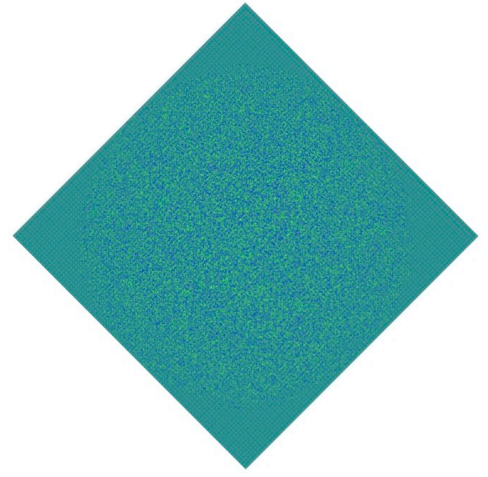

(e) Graph homomorphism into $\mathbb{Z}$ (the model studied in this article).

Figure 1: Examples of limit shapes in several models. This article studies the model from Figure 1e. The other models are included as illustrations of the universality of the limit shape phenomenon. Each subfigure shows a typical microscopic state for the specified model.

Figure 1 shows typical configurations for some of these models, and some aspects of their limit shapes are already visible, such as the arctic circle (cf. [JPS98]) in Figure 1a and its analogues in the other figures. Figure 1e is a configuration of the model studied in this article, which we briefly introduce here (see Section 2 for a formal presentation of the model). We take as given a sequence of finite subsets $R_{n} \subset \mathbb{Z}^{m}$, such that the rescaled sequence $\left(\frac{1}{n} R_{n}\right)_{n \in \mathbb{N}}$ converges to a suitable domain $R \subset \mathbb{R}^{m}$. The basic objects in the model are graph homomorphisms $h_{R_{n}}: R_{n} \rightarrow \mathbb{Z}$, which we call $\mathbb{Z}$-homomorphisms or height functions, and the limit objects are Lipschitz continuous maps $h_{R}: R \rightarrow \mathbb{R}$, called asymptotic height functions.

The main results of this article partially describe the asymptotic structure of the set of height functions on $R_{n}$ subject to certain boundary constraints. The focus is to measure how may height functions are close to the various admissible asymptotic height functions 
(after rescaling), i.e. the size of the ball

$$
B\left(R_{n}, h_{R}, \varepsilon\right):=\left\{h_{R_{n}}: R_{n} \rightarrow \mathbb{Z}\left|\sup _{z \in R_{n}}\right| \frac{1}{n} h_{R_{n}}(z)-h_{R}\left(\frac{1}{n}\right) \mid<\varepsilon\right\},
$$

and to determine for which asymptotic height functions $h_{R}$ the balls $B\left(R_{n}, h_{R}, \varepsilon\right)$ are largest. We use the following definitions, well-known from statistical physics, to state the main results: For a set of height functions $A \subset\left\{h_{R_{n}}: R_{n} \rightarrow \mathbb{Z}\right\}$, the microscopic entropy $\operatorname{Ent}_{R_{n}}(A)$ is given by

$$
\operatorname{Ent}_{R_{n}}(A):=-\frac{1}{\left|R_{n}\right|} \log |A| .
$$

The macroscopic entropy of an asymptotic height function $h_{R}: R \rightarrow \mathbb{R}$ is given by

$$
\operatorname{Ent}_{R}\left(h_{R}\right):=\int_{R} \operatorname{ent}\left(\nabla h_{R}(x)\right) d x
$$

where ent $:[-1,1]^{m} \rightarrow \mathbb{R}$ is the local surface tension, a real-valued function of the height function gradient determined entirely by the specifics of the chosen model. (The Lipschitz condition satisfied by asymptotic height functions is such that their gradients lie in $[-1,1]^{m}$; see Definition 4.) Note that the local surface tension for a general height function model admits several equivalent definitions (see e.g. [She05, Chapters 4 and 6]), but this definition is easiest for our purposes.

As an aside, the $\mathbb{Z}$-homomorphism model in two dimensions is equivalent to the six vertex model with uniform weights. Recall that a configuration of the six vertex is an assignment of one of six admissible states to each vertex in a specific subset of the lattice $\mathbb{Z}^{2}$, subject to certain local compatibility conditions. Weights $w_{1}, \ldots, w_{6}$ are associated to the six possible vertex states, and at least in the case of finite volume, configurations are sampled in proportion to the product of the weights of the vertices. When the weights are uniform (i.e. $w_{1}=\cdots=w_{6}=1$ ) then the induced measure is uniform over admissible configurations, and the related partition function counts the number of configurations. If one follows the conventions of [RS18], then each configuration of the six vertex model has a unique (up to additive constant) associated height function, defined on the faces of the lattice, such that the heights of two adjacent faces differ by \pm 1 . This height function is a $\mathbb{Z}$-homomorphism defined on the dual lattice. (Note that there is another common convention used to define height functions for the six vertex model, used in [BCG16] among others; a review of the six vertex model is beyond the scope of this article.) A configuration of the six-vertex model is shown in Figure 2, along with its associated height function. Note that the six vertex configuration satisfies the well-studied domain wall boundary conditions, and (therefore) the height function has extremal slope along all four edges of the boundary. As mentioned before, the partition function of the six vertex model counts the number of configurations (because we take uniform weights). Since the configurations are in bijection with their height functions, the six vertex partition function is closely related to the microscopic entropy defined above. As such, the results of this article can be translated to corresponding results for the six vertex model, with uniform weights and appropriately translated boundary conditions. 


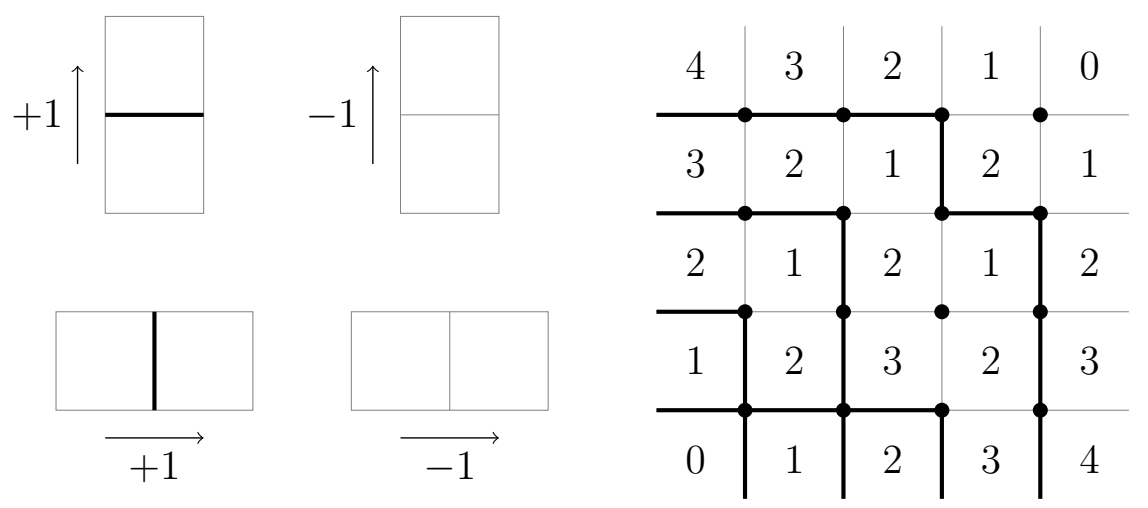

Figure 2: The height functions rules for the six vertex model (cf. [RS18, Figure 4]), and an example of a configuration of the six vertex model and the associated height function. The results of this article imply the variational principle and large deviations principle for the six vertex model with uniform weights and any boundary data. The example above shows the domain wall boundary data, which corresponds to a boundary height function with extremal slope along all four edges of the square.

Now we are prepared to state two of the three main results of this article: the profile theorem and the variational principle. The profile theorem (Theorem 15) holds that, given an asymptotic height function $h_{R}$,

$$
\operatorname{Ent}_{R_{n}}\left(B\left(R_{n}, h_{R}, \varepsilon\right)\right) \approx \operatorname{Ent}_{R}\left(h_{R}\right) \quad \text { as } n \rightarrow \infty \text { and } \varepsilon \rightarrow 0 .
$$

The conclusion of the variational principle (Theorem 16) is essentially that, given boundary data $h_{\partial R}: \partial R \rightarrow \mathbb{R}$

$$
\begin{aligned}
& \operatorname{Ent}_{R_{n}}(\left.\left\{h_{R_{n}}: R_{n} \rightarrow \mathbb{Z}\left|\sup _{z \in \partial R_{n}}\right| \frac{1}{n} h_{R_{n}}(z)-h_{\partial R}\left(\frac{1}{n} z\right) \mid<\varepsilon\right\}\right) \\
& \approx \inf _{\substack{h_{R}:\left.R \rightarrow \mathbb{R} \\
h_{R}\right|_{\partial R}=h_{\partial R}}} \operatorname{Ent}_{R}\left(h_{R}\right) \text { as } \varepsilon \rightarrow 0 .
\end{aligned}
$$

(Note that a few technical corrections are required in (2), since e.g. the asymptotic boundary height function $h_{\partial R}$ is generally not defined at rescaled lattice points $\frac{1}{n} z, z \in$ $R_{n} \subset \mathbb{Z}^{m}$; see Theorem 16 for the more precise statement.)

The third main result is the large deviations principle. There is a conventional notation used in the study of large deviations, which we use in the statement of Theorem 17 below. But, for consistency with asymptotic identities (1) and (2), we can restate the large deviations principle as follows: Given a suitable set of asymptotic height functions $A \subset\left\{h_{R}: R \rightarrow \mathbb{R}\right\}$,

$$
\begin{aligned}
\operatorname{Ent}_{R_{n}}( & \left.\left\{h_{R_{n}}: R_{n} \rightarrow \mathbb{Z} \mid \text { after rescaling, } h_{R_{n}} \in A\right\}\right) \\
& \approx \inf _{h_{R} \in A} \operatorname{Ent}_{R}\left(h_{R}\right) \text { as } n \rightarrow \infty
\end{aligned}
$$

When the macroscopic entropy has a unique minimizer, the large deviations principle implies that almost all microscopic states must approximate this entropy-minimizing profile. Although the current article does not prove uniqueness of this minimizer, we briefly 


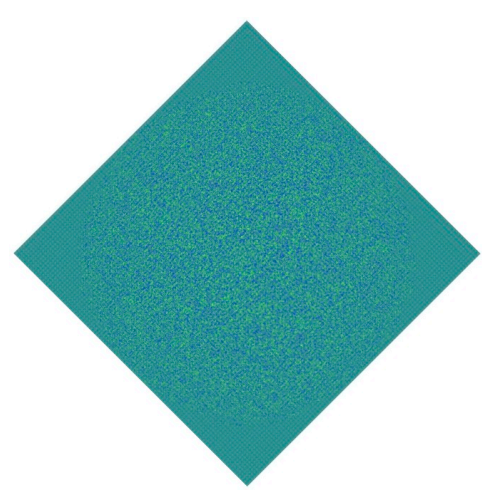

(a) Without random field.

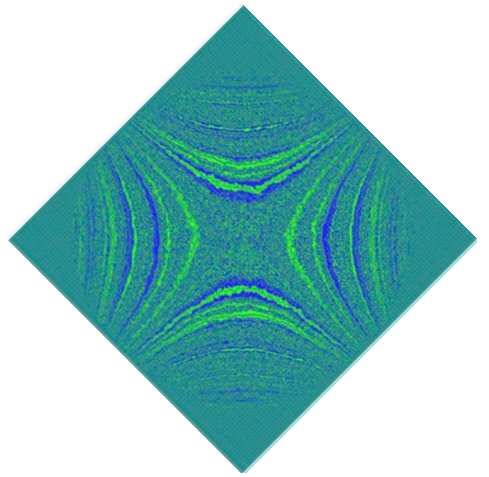

(b) Bounded random field.

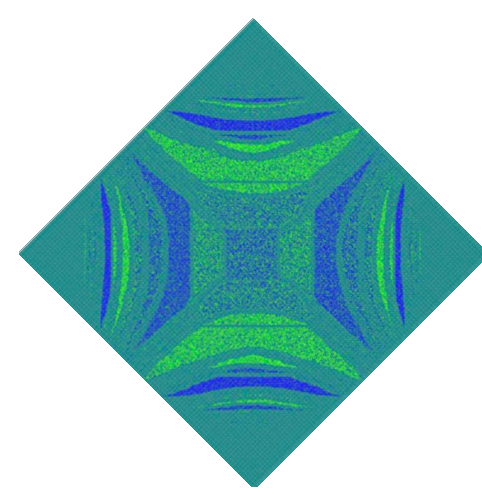

(c) Unbounded random field.

Figure 3: Quenched Aztec diamonds, 2d perspective. The configuration pictured in Figure $3 \mathrm{a}$ is equivalent to the model studied in the current article, and the others are drawn from a generalized model that the authors believe is amenable to study using the methods described in the current article.

discuss uniqueness in Section 2.5, after the statement of the large deviations principle (Theorem 17).

The results describes above are standard within the study of statistical mechanics. Indeed, the intention of this article is neither to prove novel results about the $\mathbb{Z}$ homomorphism model nor to extend well-known results to subtle and technically challenging models (as for example in [MT20]). Rather, the intention of this article is to provide and explain a simplified method of proof that only relies on minimal a priori assumptions. The simple $\mathbb{Z}$-homomorphism model is chosen as the object of study in order to avoid unnecessary technical difficulties that might arise in more complex models.

Our method of proof emerges from distilling the core arguments of [CKP01, She05, MT20]. Compared to those works, our method does not rely on explicit formulas for the local surface tension, strict convexity, concentration inequalities, or the FKG inequality. The robustness of this method is illustrated in the companion article [KMT18]. There, we show the homogenization of the variational principle of graph homomorphisms to $\mathbb{Z}$. In homogenization, homomorphisms are not chosen according to the uniform measure but instead certain heights are preferred or penalized according to a random field. Mathematically the height function is sampled from a Gibbs measure with respect to a randomized Hamiltonian. The limit shape may change drastically; for example, when the random field is unbounded, simulations show the formation of terraces. See Figures 3 and 4 for examples. We hope that the method outlined in this article can serve as a guiding principle for deducing the variational principle and related results for more complex models.

As hinted above, the three main results of this article are the profile theorem (Theorem 15), the variational principle (Theorem 16), and the large deviations principle (Theorem 17). The majority of the effort in this article goes into proving the profile theorem. The proof starts by proving the profile theorem in a special case (where the domain $R$ is the union of simplices and the limiting profile $h_{R}$ is piecewise affine), then bootstraps 


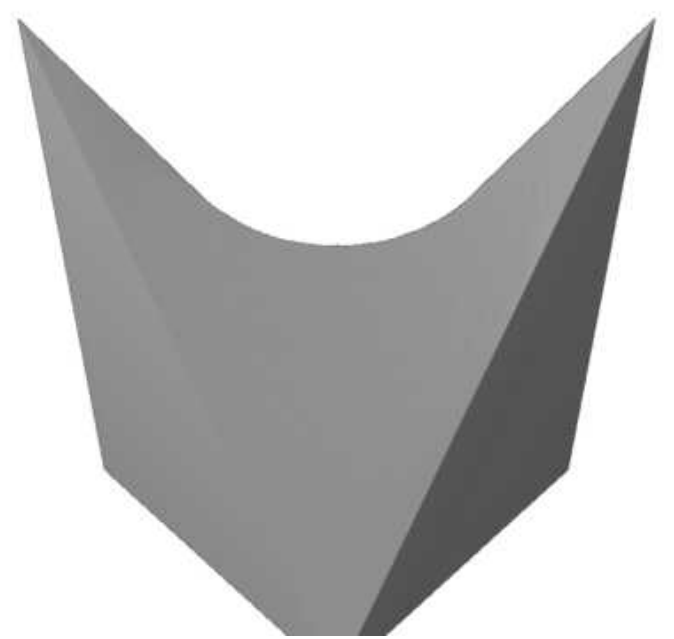

(a) The height function from Figure 3a, which was sampled without perturbation from a random field.

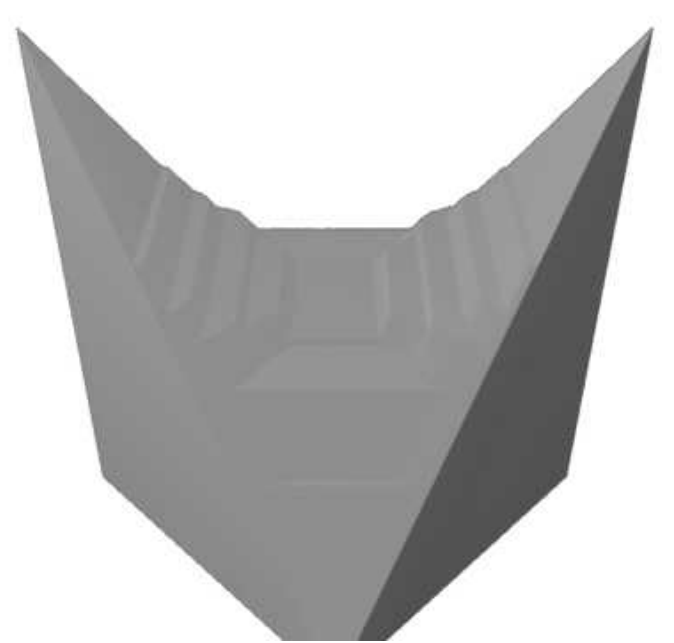

(b) The height function from Figure 3c, which was sampled with perturbation from an unbounded random field.

Figure 4: Quenched Aztec diamonds, 3d perspective. Similar to Figure 3, the first figure shows a configuration of the model studied in this article, and the second shows a configuration from a generalized model to be studied in a later work.

this result to the general case. The main idea of each step in this proof is clear, although some care is needed to account for all the details.

We call attention to two ingredients in the proof. The first ingredient is the simplicial Rademacher theorem, so called because it approximates a Lipschitz function $h_{R}$ uniformly over a large portion of its domain by a piecewise affine approximation $h_{K}$, where the "pieces" on which $h_{K}$ is affine are simplices. This approximation gives control over both the direct error $\left|h_{K}-h_{R}\right|$ and over the error in the derivatives $\left|\nabla h_{K}-\nabla h_{R}\right|$. Compared to the classical Rademacher theorem which states that $h_{R}$ is almost everywhere differentiable, the simplicial Rademacher theorem is a surprisingly strong approximation result. This seems like a standard result but the authors have not found it stated in this form in the random surfaces literature, so we give details of the proof.

In order to exploit the simplicial Rademacher theorem, we need robustness of both the macroscopic and microscopic entropy, under changes both to the limiting profile and to the domain. Robustness of the macroscopic entropy follows from elementary analysis, because the simplicial Rademacher approximation has derivative $\nabla h_{K}$ close to $\nabla h_{R}$. Robustness of the microscopic entropy rests largely upon the second ingredient that we call attention to: a Kirszbraun theorem for graph homomorphisms (see Theorem 19). This theorem gives conditions under which a height function $h_{R_{n}}: R_{n} \rightarrow \mathbb{Z}$ may be extended to a larger domain $\tilde{R}_{n} \supseteq R_{n}$. We expect that the main challenge in extending the method of this article to other models will be proving a comparable extension theorem.

The profile theorem can be used to prove the variational principle and the large devi- 
ations principle. Both proofs are similar, and rely on the local compactness of the space of Lipschitz functions. Moreover the two proofs are robust; once the profile theorem is proven for a model (with suitable macroscopic state space), the variational principle and large deviations principle follow automatically. We present the proof of the variational principle first and in greater detail. For the large deviations principle we highlight the differences in proof, and we also change notation (replacing symbols like Ent ${ }_{n}$ and Ent), in order to match the conventions of large deviations theory.

\section{Overview of remaining article}

In Section 2 we define the setting and formulate the main results of this article. In Section 3 we explain the main idea and the structure of the proofs of the main results. The details are then given in Sections 4 through 8.

\section{Notation}

- $x$ and $y$ usually denote points in $\mathbb{R}^{m}$.

- $z$ usually denotes a point in $\mathbb{Z}^{m}$.

- For $x \in \mathbb{R},\lfloor x\rfloor$ denotes the largest integer $\leqslant x$, and $\lceil x\rceil$ denotes the smallest integer $\geqslant x$.

- For $x=\left(x_{1}, \ldots, x_{m}\right) \in \mathbb{R}^{m},\lfloor x\rfloor:=\left(\lfloor x\rfloor_{1}, \ldots,\lfloor x\rfloor_{m}\right)$.

- Given a set $A$ in some topological space, $A^{\circ}$ and $\bar{A}$ denote the interior and closure of $A$ respectively.

- $R$ and $R_{n}$ are "nice" domains in $\mathbb{R}^{m}$ and $\mathbb{Z}^{m}$ respectively (see Assumption 2 below).

- $h_{R}: R \rightarrow \mathbb{R}$ is an asymptotic height function (i.e. 1-Lipschitz function).

- $h_{R_{n}}: R_{n} \rightarrow \mathbb{Z}$ is a height function (i.e. graph homomorphism).

- $\theta(\delta)$ denotes a function with $\lim _{\delta \downarrow 0} \theta(\delta)=0$ (see Section 2.4).

- $\theta_{a, b, c}(\delta)$ denotes a function with $\lim _{\delta \downarrow 0} \theta_{a, b, c}(\delta)=0$, with rate of convergence depending only on the parameters $a, b, c$.

- For a set $A$ we denote with $|A|$ either the cardinality of $A$ or the Lebesgue measure of $A$. 


\section{$2 \quad$ Setting and main results}

In this section we formally describe the model under study, and state the main results that we prove. In describing the model we err on the side of verbosity and explicitness. Some of the notations used are non-standard (such as the $\theta$-notation for asymptotics described in Section 2.4), but these notations allow for relatively concise and (more importantly) precise statements of the results and proofs to follow.

In Section 2.1 we will carefully introduce the basic model, i.e. height functions on "nice" subsets of $\mathbb{Z}^{m}$. In Section 2.2 we describe a canonical family of height functions. In Section 2.3 we use these canonical height functions to define the microscopic entropy, then we go on to define the macroscopic entropy and surface tension. In Section 2.4 we introduce our asymptotic notation, as mentioned above. In Section 2.5 the main results of this article are stated.

\subsection{Objects of study}

Given a graph $\Gamma=(V, E)$, we write " $v_{1} \sim v_{2}$ " if two vertices $v_{1}, v_{2} \in V$ are adjacent; i.e. if $\left\{v_{1}, v_{2}\right\} \in E$. Given two graphs $\Gamma_{1}=\left(V_{1}, E_{1}\right)$ and $\Gamma_{2}=\left(V_{2}, E_{2}\right)$, we recall that a graph homomorphism is a function $\varphi: V_{1} \rightarrow V_{2}$ such that whenever vertices $v, v^{\prime} \in V_{1}$ are adjacent in $\Gamma_{1}$, their images $\varphi(v), \varphi\left(v^{\prime}\right) \in V_{2}$ are adjacent in $\Gamma_{2}$.

In this article, we only consider to the case of graph homomorphisms from certain subgraphs $R_{n} \subset \mathbb{Z}^{m}$ to a subgraph of $\mathbb{Z}$. For $R_{n} \subset \mathbb{Z}^{m}$, we write $R_{n}^{c}$ for the complement of $R_{n}$ in $\mathbb{Z}^{m}$, and $\partial R_{n}:=\left\{z \in R_{n} \mid \exists z^{\prime} \in R_{n}^{c}, z \sim z^{\prime}\right\}$ for the (inner) boundary of $R_{n}$.

The graph $\mathbb{Z}^{m}$ is bipartite, and for concreteness and consistency we label the two parts of $\mathbb{Z}^{m}$ by parity. Specifically, a vertex $z=\left(z_{1}, \ldots, z_{m}\right) \in \mathbb{Z}^{m}$ is even if $z_{1}+\cdots+z_{m}$ is even, and $z$ is odd otherwise. Every edge $e \in E\left(\mathbb{Z}^{m}\right)$ is incident to one even vertex and one odd vertex. Moreover, every graph homomorphism $h$ from a connected domain $R_{n} \subset \mathbb{Z}^{m}$ to $\mathbb{Z}$ either preserves parity at every point (i.e. $h(z)$ has the same parity as $z$ has) or inverts parity at every point. This is relevant when extending a homomorphism from a disconnected domain $\tilde{R}_{n} \subset \mathbb{Z}^{m}$ to a larger connected domain, since if $h$ preserves parity on one component of $\tilde{R}_{n}$ and inverts parity on another component, then no extension is possible; cf. the Kirszbraun theorem (Theorem 19).

For simplicity we restrict our attention to parity preserving homomorphisms on connected domains. To extend to general homomorphisms on connected domains, one can use a bijection between the set of parity preserving homomorphisms and the set of parity inverting homomorphisms, e.g. the map $h \mapsto(z \mapsto h(z)+1)$. For disconnected domains $S \subset \mathbb{Z}^{m}$, the choice of parity on each component of $S$ contributes to the entropy, so the results become more delicate. We will not consider these generalizations further in this article.

Definition 1. A height function on $R_{n}$ is a graph homomorphism $h_{R_{n}}: R_{n} \rightarrow \mathbb{Z}$ that 
preserves parity, meaning that for $z=\left(z_{1}, \ldots, z_{m}\right) \in \mathbb{Z}^{m}$,

$$
h_{R_{n}}(z) \bmod 2=z \bmod 2\left(:=\left[\sum_{i=1}^{m} z_{i}\right] \bmod 2\right) .
$$

We call a height function $h_{\partial R_{n}}: \partial R_{n} \rightarrow \mathbb{Z}$, defined on the boundary of $R_{n}$, a boundary height function.

We are interested in sequences of subgraphs $\left\{R_{n} \mid n \in \mathbb{N}\right\}$ that converge under a scaling limit to a "nice" region $R \subset \mathbb{R}^{m}$. More specifically, we make the following assumptions.

Assumption 2. We assume that $R \subset \mathbb{R}^{m}$ is compact and connected and that $R$ is the closure of its interior (sets with the latter property are called regular closed sets; see e.g. [SS95]).

We assume that $R_{n} \subset \mathbb{Z}^{m}$ and $\partial R_{n} \subset R$ are connected as subgraphs of $\mathbb{Z}^{m}$, and (for simplicity) we assume that $\frac{1}{n} R_{n} \subset R$.

We require that $\frac{1}{n} R_{n} \rightarrow R$ in the Hausdorff metric; that is, the metric on $\mathcal{P}\left(\mathbb{R}^{m}\right):=$ $\left\{A \subset \mathbb{R}^{m}\right\}$ defined by

$$
d_{H}(A, B):=\left(\sup _{x \in A} \inf _{y \in B}|x-y|_{1}\right) \vee\left(\sup _{y \in B} \inf _{x \in A}|x-y|_{1}\right) .
$$

Remark 3. By equivalence of norms, it does not matter which norm on $\mathbb{R}^{m}$ is used in (3). Later, we will be interested primarily in the $\ell^{1}$ norm. This is because the $\ell^{1}$ norm is the scaling limit of the graph distance on $\mathbb{Z}^{m}$. More precisely, if $x, x^{\prime} \in \mathbb{R}^{m}$ and if $z_{n}, z_{n}^{\prime} \in \mathbb{Z}^{m}$ satisfy $\left|\frac{1}{n} z_{n}-x\right|_{1}<\frac{m}{n}$ and $\left|\frac{1}{n} z_{n}^{\prime}-x^{\prime}\right|_{1}<\frac{m}{n}$, then $\frac{1}{n} d_{\mathbb{Z}^{m}}\left(z_{n}, z_{n}^{\prime}\right) \rightarrow\left|x-x^{\prime}\right|_{1}$, where $d_{\mathbb{Z}^{m}}$ denotes the graph distance.

For example, when $R$ is compact, convex polytope, such as a hypercube or a simplex, the sets $R_{n}:=\left\{z \in \mathbb{Z}^{m} \mid \frac{1}{n} z \in R\right\}$ satisfy Assumption 2 .

Just as the microscopic domains $R_{n}$ have a scaling limit, so do the microscopic height functions $h_{R_{n}}: R_{n} \rightarrow \mathbb{Z}$.

Definition 4. We call a function $h_{R}: R \rightarrow \mathbb{R}$ an asymptotic height function if $h_{R}$ is Lipschitz with Lipschitz constant at most 1 , with respect to the $\ell^{1}$-norm on $\mathbb{R}^{m}$; that is, if

$$
\operatorname{Lip}\left(h_{R}\right):=\sup _{x \neq y \in R} \frac{\left|h_{R}(x)-h_{R}(y)\right|}{|x-y|_{1}} \leqslant 1 .
$$

Likewise, if $h_{\partial R}: \partial R \rightarrow \mathbb{R}$ is 1-Lipschitz (with respect to the $\ell^{1}$-norm), we call $h_{\partial R}$ an asymptotic boundary height function.

We assume that $h_{\partial R_{n}}: \partial R_{n} \rightarrow \mathbb{Z}$ are boundary height functions that converge (after rescaling) to an asymptotic boundary height function $h_{\partial R}: \partial R \rightarrow \mathbb{R}$ in following sense: for each $n$, let $d_{n}=d_{H}\left(\frac{1}{n} R_{n}, R\right)$. Then, we say $\frac{1}{n} h_{\partial R_{n}} \rightarrow h_{\partial R}$ if and only if

$$
\lim _{n \rightarrow \infty} \sup _{z \in \partial R_{n}} \sup _{\substack{x \in \partial R \\\left|x-\frac{1}{n} z\right|_{1} \leqslant d_{n}}}\left|\frac{1}{n} h_{\partial R_{n}}(z)-h_{\partial R}(x)\right|=0 .
$$


Now, we define a few families of height functions and asymptotic height functions. The sets of height functions from Definition 5 below appear frequently in entropies, and the sets of asymptotic height functions from Definition 6 are important for the statement of the variational principle (Theorem 16).

Definition 5. Let $R_{n}$ be a microscopic domain as above, let $h_{R_{n}}: R_{n} \rightarrow \mathbb{Z}$ be a boundary height function, and let $\delta>0$. We define:

$$
\begin{aligned}
M\left(R_{n}\right) & :=\left\{h_{R_{n}}: R_{n} \rightarrow \mathbb{R} \mid h_{R_{n}} \text { is a height function }\right\}, \\
M\left(R_{n}, h_{\partial R_{n}}\right) & :=\left\{h_{R_{n}} \in M\left(R_{n}\right)\left|h_{R_{n}}\right| \partial R_{n}=h_{\partial R_{n}}\right\} \\
M\left(R_{n}, h_{\partial R_{n}}, \delta\right) & :=\left\{h_{R_{n}} \in M\left(R_{n}\right)\left|\sup _{z \in \partial R_{n}}\right| h_{R_{n}}(z)-h_{\partial R_{n}}(z) \mid<\delta n\right\}, \text { and } \\
B\left(R_{n}, h_{R}, \delta\right) & :=\left\{h_{R_{n}} \in M\left(R_{n}\right)\left|\sup _{z \in R_{n}}\right| h_{R}\left(\frac{1}{n} z\right)-\frac{1}{n} h_{R_{n}}(z) \mid<\delta\right\} .
\end{aligned}
$$

In the last definition, the expression " $h_{R}\left(\frac{1}{n} z\right)$ " makes sense because of the assumption that $\frac{1}{n} R_{n} \subset R$ in Assumption 2 .

Definition 6. Let $R \subset \mathbb{R}^{m}$ be a domain satisfying Assumption 2 , let $h_{\partial R}: \partial R \rightarrow \mathbb{R}$ be an asymptotic boundary height function, and let $\delta>0$. We define:

$$
\begin{aligned}
M(R) & :=\left\{h_{R}: R \rightarrow \mathbb{R} \mid h_{R} \text { is an asymptotic height function }\right\} \\
M\left(R, h_{\partial R}\right) & :=\left\{h_{R}: R \rightarrow \mathbb{R}\left|h_{R}\right|_{\partial R}=h_{\partial R}\right\}, \text { and } \\
M\left(R, h_{\partial R}, \delta\right): & =\left\{h_{R}: R \rightarrow \mathbb{R}|\forall x \in \partial R,| h_{R}(x)-h_{\partial R}(x) \mid \leqslant \delta\right\} .
\end{aligned}
$$

\subsection{Affine height functions}

Affine height functions play an important role in defining and studying the entropy of our model. For an asymptotic height function $h_{R}: R \rightarrow \mathbb{R}$, we mean by "affine" the usual property: there exist $s \in[-1,1]^{m}$ and $b \in \mathbb{R}$ such that $h_{R}(x)=s \cdot x+b$. The bounds on $s$ ensure that $h_{R}$ satisfies the Lipschitz property (4), so all such functions are indeed asymptotic height functions as per Definition 4.

On microscopic domains $R_{n}$, we consider best-possible approximations to affine functions. Fix $s \in[-1,1]^{m}$ and $b \in \mathbb{R}$. At a lattice point $z \in \mathbb{Z}^{m}$, we define $h_{R_{n}}^{s \cdot x+b}(z)$ to be $s \cdot z+b$, rounded to the nearest integer of correct parity (see Figure 5). In the rest of this subsection, we formalize this definition, verify that it actually does define a height function, and check that it is consistent.

Let us introduce an auxiliary notation that is used only in this subsection. Given a point $z=\left(z_{1}, \ldots, z_{m}\right) \in \mathbb{Z}^{m}$, we say $z$ has even or odd parity as $\left(\sum_{i=1}^{m} z_{i}\right) \in \mathbb{Z}$ has even or odd parity respectively, and we write $z \bmod 2$ for the parity of $z$.

Given $z \in \mathbb{Z}^{m}$ and $y \in \mathbb{R}$, we write $[y]_{z} \bmod 2$ for the closest integer to $y$ that has parity $z \bmod 2$. In case of a tie, i.e. if $y$ is an integer that has opposite parity to $z$, we arbitrarily choose to "round up" and set $[y]_{z \bmod 2}=y+1 \in \mathbb{Z}$. 


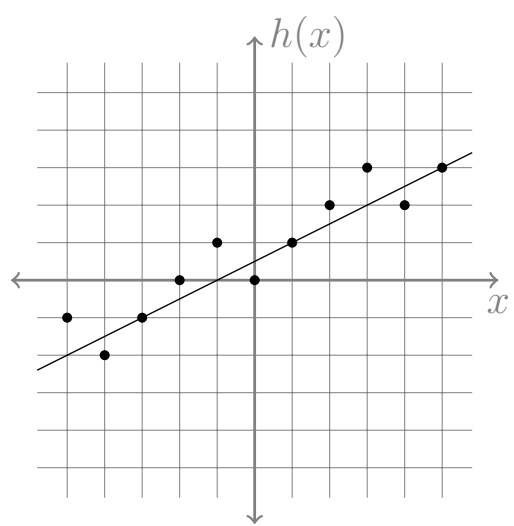

Figure 5: An affine height function $h_{R_{n}}^{s \cdot x+b}$ and the corresponding continuous affine function $x \mapsto s \cdot x+b$. Here $s=\frac{1}{2}$ and $b=\frac{1}{2}$.

For example, let $z=(1,2,3) \in \mathbb{Z}^{3}$ and $z^{\prime}=(4,-6,7)$. Then $z$ is an even point and $z^{\prime}$ is an odd point. So:

$$
\begin{aligned}
{[5.4]_{z \bmod 2} } & =6, & {[-3]_{z \bmod 2} } & =-2, \\
{[5.4]_{z^{\prime} \bmod 2} } & =5, & {[-3]_{z^{\prime} \bmod 2} } & =-3 .
\end{aligned}
$$

Now, given $s \in[-1,1]^{m}$ and $b \in \mathbb{R}$, we define the affine height functions $h_{R_{n}}^{s \cdot x+b}$ by

$$
h_{R_{n}}^{s \cdot x+b}(z):=[s \cdot z+b]_{z \bmod 2} .
$$

Note that the symbol $x$ in the superscript of $h_{R_{n}}^{s \cdot x+b}$ is merely formal; " $s \cdot x+b$ " should be read as "the function mapping $x$ to $s \cdot x+b$ ". Moreover, the choice of domain $R_{n}$ in the subscript does not affect the values of $h_{R_{n}}^{s \cdot x+b}$ at any point; for any sets $A_{n}, B_{n} \subseteq \mathbb{Z}^{m}$ and any point $z \in A_{n} \cap B_{n}$, one has $h_{A_{n}}^{s \cdot x+b}(z)=h_{B_{n}}^{s \cdot x+b}(z)$. An example of a function $h_{R_{n}}^{s \cdot x+b}$ is provided in Figure 5.

From the definition above, it is not clear that $h_{R_{n}}^{s \cdot x+b}$ are height functions. This is the content of Lemma 7.

Lemma 7. Let $s \in[-1,1]^{m}$ and $b \in \mathbb{R}$. For any adjacent points $z \sim z^{\prime} \in \mathbb{Z}^{m}$, the values $h_{R_{n}}^{s \cdot x+b}(z)$ and $h_{R_{n}}^{s \cdot x+b}\left(z^{\prime}\right)$ differ by exactly 1 .

Proof. From the definition of $h_{R_{n}}^{s \cdot x+b}$, we note two inequalities:

$$
\left|h_{R_{n}}^{s \cdot x}(z)-(s \cdot z+b)\right| \leqslant 1
$$

and

$$
\left|h_{R_{n}}^{s \cdot x+b}\left(z^{\prime}\right)-\left(s \cdot z^{\prime}+b\right)\right| \leqslant 1 .
$$

Additionally, since $s \in[-1,1]^{m}$, we have

$$
\left|(s \cdot z+b)-\left(s \cdot z^{\prime}+b\right)\right| \leqslant 1 \text {. }
$$


By the triangle inequality, $\left|h_{R_{n}}^{s \cdot x+b}(z)-h_{R_{n}}^{s \cdot x+b}\left(z^{\prime}\right)\right| \leqslant 3$. We shall show that equality cannot hold. Since the difference $h_{R_{n}}^{s \cdot x+b}(z)-h_{R_{n}}^{s \cdot x+b}\left(z^{\prime}\right)$ is obviously an odd integer, it will follow that the difference is \pm 1 .

Suppose towards a contradiction that

$$
\left|h_{R_{n}}^{s \cdot x+b}(z)-h_{R_{n}}^{s \cdot x+b}\left(z^{\prime}\right)\right|=3 .
$$

Then (5) and (6) must be equalities. From the definition of $[\cdot]_{z} \bmod 2$, necessarily then $s \cdot z+b$ is an integer with parity opposite that of $z$, and so

$$
h_{R_{n}}^{s \cdot x+b}(z)=(s \cdot z+b)+1 .
$$

Likewise

$$
h_{R_{n}}^{s \cdot x+b}\left(z^{\prime}\right)=\left(s \cdot z^{\prime}+b\right)+1 .
$$

But then

$$
\left|h_{R_{n}}^{s \cdot x+b}(z)-h_{R_{n}}^{s \cdot x+b}\left(z^{\prime}\right)\right|=\left|(s \cdot z+b+1)-\left(s \cdot z^{\prime}+b+1\right)\right| \leqslant 1 .
$$

We end this section with the following lemma. The conclusion (8) is exactly what is needed later to apply the Kirszbraun theorem (Theorem 19):

Lemma 8. Let $s, s^{\prime} \in[-1,1]^{m}, b, b^{\prime} \in \mathbb{R}$, and $z, z^{\prime} \in \mathbb{Z}^{m}$. If

$$
\left|(s \cdot z+b)-\left(s^{\prime} \cdot z^{\prime}+b^{\prime}\right)\right| \leqslant\left|z-z^{\prime}\right|_{1},
$$

then

$$
\left|h_{\{z\}}^{s \cdot x+b}(z)-h_{\left\{z^{\prime}\right\}}^{s^{\prime} \cdot b^{\prime}}\left(z^{\prime}\right)\right| \leqslant\left|z-z^{\prime}\right|_{1} .
$$

Proof. The proof is similar to that of Lemma 7. By the triangle inequality and (7),

$$
\begin{aligned}
& \left|h_{\{z\}}^{s \cdot x+b}(z)-h_{\left\{z^{\prime}\right\}}^{s^{\prime} \cdot x+b^{\prime}}\left(z^{\prime}\right)\right| \\
& \leqslant\left|h_{\{z\}}^{s \cdot x+b}(z)-(s \cdot z+b)\right|+\left|(s \cdot z+b)-\left(s^{\prime} \cdot z^{\prime}+b^{\prime}\right)\right| \\
& \quad+\left|\left(s^{\prime} \cdot z^{\prime}+b^{\prime}\right)-h_{\left\{z^{\prime}\right\}}^{s^{\prime} \cdot x+b^{\prime}}\left(z^{\prime}\right)\right| \\
& \leqslant\left|z-z^{\prime}\right|_{1}+2 .
\end{aligned}
$$

Since $h_{\{z\}}^{s \cdot x+b}(z), h_{\left\{z^{\prime}\right\}}^{s^{\prime} \cdot x+b^{\prime}}\left(z^{\prime}\right)$ and $\left|z-z^{\prime}\right|_{1}$ are all integers,

$$
\left|h_{\{z\}}^{s \cdot x+b}(z)-h_{\left\{z^{\prime}\right\}}^{s^{\prime} \cdot x+b^{\prime}}\left(z^{\prime}\right)\right|-\left|z-z^{\prime}\right|_{1} \in\{\ldots,-2,-1,0,1,2\} .
$$

We want to prove that the left-hand side of $(10)$ is $\leqslant 0$. By parity considerations it must be even, and we need only prove it is $\neq 2$. Assume for a contradiction that the left-hand side of (10) equals 2. Then equality holds in (9), and in particular

$$
\left|h_{\{z\}}^{s \cdot x+b}(z)-(s \cdot z+b)\right|=1 \quad \text { and } \quad\left|h_{\left\{z^{\prime}\right\}}^{s^{\prime} \cdot x+b^{\prime}}\left(z^{\prime}\right)-\left(s^{\prime} \cdot z^{\prime}+b^{\prime}\right)\right|=1 .
$$

As in the proof of Lemma 7, this implies that

$$
h_{\{z\}}^{s \cdot x+b}(z)=(s \cdot z+b)+1 \quad \text { and } \quad h_{\left\{z^{\prime}\right\}}^{s^{\prime} \cdot x+b^{\prime}}\left(z^{\prime}\right)=\left(s^{\prime} \cdot z^{\prime}+b^{\prime}\right)+1 .
$$

Therefore

$$
\left|h_{\{z\}}^{s \cdot x+b}(z)-h_{\left\{z^{\prime}\right\}}^{s^{\prime} \cdot b^{\prime}}\left(z^{\prime}\right)\right|=\left|(s \cdot z+b)-\left(s^{\prime} \cdot z^{\prime}+b^{\prime}\right)\right| \leqslant\left|z-z^{\prime}\right|_{1} .
$$

This is the desired contradiction, which completes the proof. 


\subsection{Entropies and surface tensions}

In this section we make three more definitions needed for our statement of the main results. First, we define the microscopic entropy of a set of height functions. More precisely, this is the Shannon entropy of the uniform distribution over a finite set of height functions, normalized by the size of their common domain, and negated. (The negative convention is chosen so that the surface tension ent $(s)$, defined later, is convex rather than concave.) The microscopic entropy is essentially the same as the specific free energy of [She05].

Definition 9. Given a finite, non-empty set of height functions $A \subset M\left(R_{n}\right)$, we define the microscopic entropy

$$
\operatorname{Ent}_{R_{n}}(A):=-\frac{1}{\left|R_{n}\right|} \log |A| \text {. }
$$

We observe that the microscopic entropy is translation invariant:

Observation 10. Let $h_{\partial R_{n}} \in M\left(\partial R_{n}\right), h_{R} \in M(R), \delta>0$, and $c \in \mathbb{R}$. Then:

$$
\begin{aligned}
\operatorname{Ent}_{R_{n}}\left(M\left(R_{n}, h_{\partial R_{n}}+c\right)\right) & =\operatorname{Ent}_{R_{n}}\left(M\left(R_{n}, h_{\partial R_{n}}\right)\right), \\
\operatorname{Ent}_{R_{n}}\left(M\left(R_{n}, h_{\partial R_{n}}+c, \delta\right)\right) & =\operatorname{Ent}_{R_{n}}\left(M\left(R_{n}, h_{\partial R_{n}}, \delta\right)\right), \text { and } \\
\operatorname{Ent}_{R_{n}}\left(B\left(R_{n}, h_{R}+c, \delta\right)\right) & =\operatorname{Ent}_{R_{n}}\left(B\left(R_{n}, h_{R}, \delta\right)\right),
\end{aligned}
$$

where $h_{R_{n}}+c$ is the height function defined by $\left(h_{R_{n}}+c\right)(z)=h_{R_{n}}(z)+c$ for $z \in R_{n}$, and likewise $\left(h_{R}+c\right)(x)=h_{R}(x)+c$ for $x \in R$.

All of these sets, except for $M\left(R_{n}\right)$, are finite, because of the constraints they impose on $h_{R_{n}}$ and the Lipschitz property of $h_{R_{n}}$. In fact, we can say more. Let us count $M\left(R_{n}, h_{\partial R_{n}}\right)$, for some boundary height function $h_{\partial R_{n}} \in M\left(\partial R_{n}\right)$. The values of $h_{R} \in$ $M\left(R_{n}, h_{\partial R_{n}}\right)$ are fixed on $\partial R_{n}$, and for each of the $\leqslant\left|R_{n}\right|$ points $x$ in the interior of $R_{n}$, there are at most 2 admissible values for $h_{R}(x)$. Therefore $\left|M\left(R_{n}, h_{\partial R_{n}}\right)\right| \leqslant 2^{\left|R_{n}\right|}$. Similar logic holds for $M\left(R_{n}, h_{\partial} R_{n}, \delta\right)$ and $B\left(R_{n}, h_{R}, \delta\right)$. This leads to the following observation:

Observation 11. Let $h_{\partial R_{n}} \in M\left(\partial R_{n}\right), h_{R} \in M(R)$, and $\delta>0$. Then:

$$
\begin{aligned}
\operatorname{Ent}_{R_{n}}\left(M\left(R_{n}, h_{\partial R_{n}}\right)\right) & \geqslant-\log 2, \\
\operatorname{Ent}_{R_{n}}\left(M\left(R_{n}, h_{\partial R_{n}}, \delta\right)\right) & \geqslant-\log 2-\frac{\log \lceil 2 \delta n\rceil}{\left|R_{n}\right|} \\
& =-\log 2-\delta O\left(\frac{1}{n^{m-1}}\right), \text { and } \\
\operatorname{Ent}_{R_{n}}\left(B\left(R_{n}, h_{R}, \delta\right)\right) & \geqslant-\log 2-\frac{\log \lceil 2 \delta n\rceil}{\left|R_{n}\right|} \\
& =-\log 2-\delta O\left(\frac{1}{n^{m-1}}\right) .
\end{aligned}
$$

Next, we define the local surface tension. There are in general many equivalent definitions of surface tension (see for example [She05, Chapter 6]). The following definition is easiest to work with for our purposes. 
Definition 12. For $s \in[-1,1]^{m}$, the local surface tension ent $(s)$ is defined to be the limit

$$
\operatorname{ent}(s):=\lim _{n \rightarrow \infty} \operatorname{ent}_{n}(s),
$$

where $\operatorname{ent}_{n}(s)$ is defined as

$$
\operatorname{ent}_{n}(s):=\operatorname{Ent}_{Q_{n}}\left(M\left(Q_{n}, h_{\partial Q_{n}}^{s \cdot x+0}\right)\right)
$$

and where $Q_{n}=[0, n)^{m} \cap \mathbb{Z}^{m}$ is the discrete hypercube of side length $n$.

The limit (12) exists by standard subadditivity arguments; we refer the interested reader to e.g. [Dur10]. In fact, by translation invariance (see Observation 10), we may replace $h_{\partial Q_{n}}^{s \cdot x+0}$ by $h_{\partial Q_{n}}^{s \cdot x+b}$ in (13), for any $b \in \mathbb{R}$. Additionally, boundedness passes through the limit in (12). Therefore:

Observation 13. For any $s \in[-1,1]^{m}$,

$$
-\log 2 \leqslant \operatorname{ent}(s) \leqslant 0
$$

Let us now define the macroscopic entropy.

Definition 14. Let $h_{R}: R \rightarrow \mathbb{R}$ denote an asymptotic height function. The macroscopic entropy $\operatorname{Ent}_{R}\left(h_{R}\right)$ is defined as

$$
\operatorname{Ent}_{R}\left(h_{R}\right):=\int_{R} \operatorname{ent}(\nabla h(x)) d x
$$

\subsection{Asymptotic notation}

In this section we introduce a notation for asymptotic error. Compared to the Landau big- $O$ notation, our $\theta$-notation abstracts away the rate of convergence of the error, but makes explicit the dependence on parameters. For this purpose we write $\theta_{\alpha}(\delta)$ for a family of unspecified functions, parameterized by a symbol $\alpha$, such that $\theta_{\alpha}(\delta) \rightarrow 0$ at a rate depending on the value of the parameter $\alpha$. That is, for any $\varepsilon>0$ and any admissible parameter value $\alpha$, there exists $\delta_{0}=\delta_{0}(\alpha)>0$ such that $0<\delta<\delta_{0}$ implies $\theta_{\alpha}(\delta)<\varepsilon$.

Extending the above notation, we frequently replace the single parameter $\alpha$ by a list of parameters $\alpha, \beta, \gamma, \ldots$ For example, we might write an identity like

$$
\begin{aligned}
& \min _{h_{R} \in M\left(R, h_{\partial R}\right)} \operatorname{Ent}_{R}\left(h_{R}\right)=\operatorname{Ent}_{R_{n}}\left(M\left(R_{n}, h_{\partial R_{n}}, \delta\right)\right) \\
&+\theta_{m, R, h_{\partial R}, R_{n}, h_{\partial R_{n}}}(\delta) \\
&+\theta_{m, R, h_{\partial R}, R_{n}, h_{\partial R_{n}}, \delta}\left(\frac{1}{n}\right)
\end{aligned}
$$

The identity states that the two entropy terms on the first line differ by a small amount; the difference vanishes as $\delta$ and $\frac{1}{n}$ go to zero, and the rate of convergence depends on several parameters. The " $\theta(\delta)$ " term depends on the parameters from the setting, namely 
the ambient dimension $m$, the region $R$, the height function $h_{R}$ of interest, and the corresponding discrete objects $R_{n}$ and $h_{R_{n}}$. The " $\theta\left(\frac{1}{n}\right)$ " term depends on these parameters along with the value of $\delta$. We find that listing out the setting parameters $m, R, h_{R}, R_{n}$, and $h_{R_{n}}$ makes the expression harder to read. So for the rest of the article we suppress these parameters from the subscripts of $\theta$ terms. Under this convention (14) becomes:

$$
\min _{h_{R} \in M\left(R, h_{\partial R}\right)} \operatorname{Ent}_{R}\left(h_{R}\right)=\operatorname{Ent}_{R_{n}}\left(M\left(R_{n}, h_{\partial R_{n}}, \delta\right)\right)+\theta(\delta)+\theta_{\delta}\left(\frac{1}{n}\right) .
$$

As mentioned above, the advantage of our $\theta$ notation is that it abstracts away the exact rates of convergence, but leaves explicit the dependencies between parameters. For example, suppose we want to make the error in approximation in (14) to be less than $\varepsilon$. We should first choose $\delta$ so that (say) $\theta(\delta)<\frac{1}{2} \varepsilon$, then choose $n$ depending on $\delta$ (and on the suppressed parameters $m, R$, etc.) so that $\theta_{\delta}\left(\frac{1}{n}\right)<\frac{1}{2} \varepsilon$.

\subsection{Main results}

The main results of this article are the profile theorem, the variational principle, and the large deviations principle:

Theorem 15 (Profile theorem). For any $h_{R} \in M(R), \delta>0$, and $n \in \mathbb{N}$,

$$
\operatorname{Ent}_{R}\left(h_{R}\right)=\operatorname{Ent}_{R_{n}}\left(B\left(R_{n}, h_{R}, \delta\right)\right)+\theta_{h_{R}}(\delta)+\theta_{h_{R}, \delta}\left(\frac{1}{n}\right) .
$$

The second main result is the variational principle:

Theorem 16 (Variational principle). For any $\delta>0$ and $n \in \mathbb{N}$,

$$
\inf _{h_{R} \in M\left(R, h_{\partial R}\right)} \operatorname{Ent}_{R}\left(h_{R}\right)=\operatorname{Ent}_{R_{n}}\left(M\left(R_{n}, h_{\partial R_{n}}, \delta\right)\right)+\theta(\delta)+\theta_{\delta}\left(\frac{1}{n}\right) .
$$

Finally, we prove a large deviations principle for the model. We adopt the conventions of large deviations (see for example [DZ09, RAS15]).

Theorem 17 (Large deviations principle). Consider the space $M(R)$ of asymptotic height functions (i.e. Lipschitz functions with Lipschitz constant $\leqslant 1$ ), endowed with the topology of uniform convergence (induced by the supremum norm).

For $\delta>0$ and $n \in \mathbb{N}$, define a probability measure $\mu_{\delta, n}$ on $M(R)$ by

$$
\mu_{\delta, n}(A):=\frac{1}{\left|M\left(R_{n}, h_{\partial R_{n}}, \delta\right)\right|}\left|\left\{h_{R_{n}} \in M\left(R_{n}, h_{\partial R_{n}}, \delta\right) \mid \tilde{h}_{R_{n}} \in A\right\}\right|,
$$

where $\tilde{h}_{R_{n}}$ is the Lipschitz function given by rescaling and interpolating $h_{R_{n}}$ so as to make it an asymptotic height function, i.e. for $z \in R_{n}, \tilde{h}_{R_{n}}\left(\frac{1}{n} z\right)=\frac{1}{n} h_{R_{n}}(z)$.

The measures $\left(\mu_{\delta, n}\right)_{\delta>0, n \in \mathbb{N}}$ satisfy a large deviations principle with speed $r_{\delta, n}:=\left|R_{n}\right|$ and tight rate function $I: M(R) \rightarrow[0, \infty]$ given by

$$
I\left(h_{R}\right):= \begin{cases}\operatorname{Ent}_{R}\left(h_{R}\right)-E & \text { if }\left.h_{R}\right|_{\partial R}=h_{\partial R}, \\ \infty & \text { otherwise. }\end{cases}
$$


where $E:=\inf _{h_{R} \in M\left(R, h_{\partial R}\right)} \operatorname{Ent}_{R}\left(h_{R}\right)$. More precisely, for any Borel subset $A \subset M(R)$,

$$
-\inf _{h_{R} \in A^{\circ}} I\left(h_{R}\right) \leqslant \frac{1}{r_{\delta, n}} \log \underline{\lim _{\delta \rightarrow 0}} \underset{n \rightarrow \infty}{\lim } \mu_{\delta, n}(A)
$$

and

$$
\frac{1}{r_{\delta, n}} \log \varlimsup_{\delta \rightarrow 0} \varlimsup_{n \rightarrow \infty} \mu_{\delta, n}(A) \leqslant-\inf _{h_{R} \in \bar{A}} I\left(h_{R}\right),
$$

where as usual $\underline{\lim }$ and $\varlimsup$ lim denote the limit inferior and superior respectively.

Remark 18. It is straightforward to reduce the double limits in Theorem 17 to (single) sequential limits, which are more common in large deviations theory. For example, one may choose any sequences $\left(\delta_{k}\right)_{k \in \mathbb{N}},\left(\varepsilon_{k}\right)_{k \in \mathbb{N}}$ such that $\delta_{k} \rightarrow 0$ and $\varepsilon_{k} \rightarrow 0$ as $k \rightarrow \infty$. Then, choose $n_{k}$ large enough that

$$
\varliminf_{n \rightarrow \infty} \mu_{\delta_{k}, n}(A)-\varepsilon_{k} \leqslant \mu_{\delta_{k}, n_{k}}(A) \leqslant \varlimsup_{n \rightarrow \infty} \mu_{\delta_{k}, n}(A)+\varepsilon_{k}
$$

and define $\mu_{k}:=\mu_{\delta_{k}, n_{k}}$ and $r_{k}:=r_{\delta_{k}, n_{k}}$. Then

$$
-\inf _{h_{R} \in A^{\circ}} I\left(h_{R}\right) \leqslant \underline{\lim } \frac{1}{r_{k}} \log \mu_{k}(A) \leqslant \varlimsup_{k \rightarrow \infty} \frac{1}{r_{k}} \log \mu_{k}(A) \leqslant-\inf _{h_{R} \in \bar{A}} I\left(h_{R}\right) .
$$

For the study of limit shapes, it is useful to prove two additional results: existence and uniqueness of the minimizer of the rate function from the large deviations principle, i.e. there exists a unique $h_{R}^{\min } \in M\left(R, h_{\partial R}\right)$ such that

$$
I\left(h_{R}^{\min }\right)=\inf _{h_{R} \in M\left(R, h_{\partial R}\right)} I\left(h_{R}\right) .
$$

Indeed, this holds for the simple model studied in the current article. See for example [She05] for proofs and discussion of these results. Even in more subtle models, the existence of the minimizer is often easy to show: the proof is standard as long as the local surface tension is convex and bounded below. To show uniqueness is harder. Uniqueness of the minimizer may be proved using strict convexity of the local surface tension; see for example [DSS08, Proposition 4.5]. We do not prove these results in the current article, but rather focus on the variational principle and large deviations principle.

Once existence and uniqueness of the minimizer are established (or in the language of the current model, once it is known that the macroscopic entropy functional admits a unique minimizing height function), one can explain the appearance of a limit shape in the following way. The set of asymptotic height functions that lie within distance $\varepsilon$ of this minimizer is an open ball in the space $M(R)$. By applying the large deviations principle on the set-theoretical complement, one sees that the percentage of microscopic height functions in $M\left(R_{n}, h_{\partial R_{n}}, \delta\right)$ that do not lie $\varepsilon$-close to the minimizer decays exponentially. In other words, with high probability a randomly chosen height function is close to the minimizer, and therefore the minimizer is the limit shape. 


\section{Outline and discussion of proof of main results}

In this section we briefly outline the proof of the main results and summarize some key ideas. Then we analyze the ingredients in the proof with an eye toward extending the proof to other random surface models.

In Section 4, we provide auxiliary results including basic properties of the local surface tension and microscopic entropy. A central ingredient of the overall argument is discussed in Section 5. There we prove the profile theorem in the special case of piecewise affine asymptotic height functions. In Section 6, we extend the profile theorem to general asymptotic height functions by an approximation argument, yielding the first main result (Theorem 15). In Section 7, we use the profile theorem and a compactness argument to prove the variational principle (Theorem 16). The argument is based on compactness of the space of asymptotic height functions with fixed boundary values $M\left(R, h_{\partial R}\right)$. Finally in Section 8, we extend the proof of the variational principle in order to prove the large deviations principle (Theorem 17).

As one can see from this outline, the main idea of the argument is to reduce the proof of the profile theorem from general domains and asymptotic height functions to simpler domains and asymptotic height functions by an approximation argument. This means that the left-hand side of (15), i.e. the macroscopic entropy, and the right-hand side, i.e. the microscopic entropy, must both be robust with respect to approximations.

The macroscopic entropy is robust because ent $(s)$ is bounded and uniformly continuous, and Lipschitz functions can be approximated very well by linear interpolations on a simplex domain; see the simplicial Rademacher theorem (Lemma 31). This approximation lemma was formulated for two dimensions in [CKP01]. The result is interesting in its own right and for the convenience of the reader we state and prove it for arbitrary dimension in Section 6.

The microscopic entropy is robust under approximations because the microscopic surface tension is very robust: even with fluctuations in the boundary values and the geometry of the boundary, one still gets the same limit in (12). This result is proved in Section 4, using a Kirszbraun theorem for graph homomorphisms stated below. This theorem gives conditions under which a graph homomorphism can be extended from a smaller domain to a larger domain. This is a discrete analogue to the classical result [Kir34], which deals with Lipschitz functions defined on subsets of $\mathbb{R}^{d}$. We also note that more general forms of the Kirszbraun theorem for graph homomorphisms are known, e.g. [CPT18].

Theorem 19 (Kirszbraun theorem for $\mathbb{Z}^{\boldsymbol{m}}$ ). Let $\Lambda$ be a connected region of $\mathbb{Z}^{m}$, let $S \subset \Lambda$, and let $\bar{h}: S \rightarrow \mathbb{Z}$ be a graph homomorphism that preserves parity. There exists a graph homomorphism $h: \Lambda \rightarrow \mathbb{Z}$ such that $h=\bar{h}$ on $S$ if and only if for all $x, y$ in $S$,

$$
|\bar{h}(x)-\bar{h}(y)| \leqslant|x-y|_{1},
$$

where $|x-y|_{1}$ is the $\ell^{1}$-norm in $\mathbb{Z}^{m}$.

Remark 20. The parity condition is necessary in general; consider for example the function $\bar{h}$ defined on $\{0,2\} \subset \mathbb{Z}$ by $\bar{h}(0)=0, \bar{h}(2)=1$. The parity condition in Theorem 19 is the reason for the parity condition in Definition 1. 
Two of the authors gave a proof of a more general version of this theorem in [MT20, Theorem 4.1]. The proof is restated below for the reader's convenience. This proof is also simplified by only addressing the model from this article, where the height functions take values in $\mathbb{Z}$ rather than in a $d$-regular tree.

Proof of Theorem 19. Obviously if an extension $h$ of $\bar{h}$ exists, then $\bar{h}$ satisfies (18). So, suppose instead that (18), and let us prove that an extension $h$ exists. For $y \in \Lambda$, set

$$
h(y):=\max \left\{\bar{h}(x)-|x-y|_{1} \mid x \in S\right\} .
$$

We must check two things: first that $h(y)=\bar{h}(y)$ when $y \in S$, and second that $|h(y)-h(\tilde{y})|=1$ when $y \sim \tilde{y}$ are adjacent points in $\Lambda$.

To prove that $\left.h\right|_{S}=\bar{h}$, let $y \in S$ and consider any point $x \in S$. By the Lipschitz property of $\bar{h}$,

$$
\bar{h}(x)-\bar{h}(y) \leqslant|\bar{h}(x)-\bar{h}(y)| \leqslant|x-y|_{1},
$$

so $\bar{h}(x)-|x-y|_{1} \leqslant \bar{h}(y)$. Therefore the maximum in (19) is attained when $x=y$, so $h(y)=\bar{h}(y)+|y-y|_{1}=\bar{h}(y)$.

To prove that $h$ is a graph homomorphism, let $y \sim \tilde{y}$ be adjacent points in $\Lambda$, and let $x, \tilde{x}$ be points in $S$ that attain the maximum in (19) for $y, \tilde{y}$ respectively, i.e. $h(y)=$ $\bar{h}(x)-|x-y|_{1}$ and $h(\tilde{y})=\bar{h}(\tilde{x})-|\tilde{x}-\tilde{y}|_{1}$. Then

$$
\begin{aligned}
h(y) & =\max \left\{\bar{h}(z)+|z-y|_{1} \mid z \in S\right\} \\
& \geqslant \bar{h}(\tilde{x})-|\tilde{x}-y| \\
& \geqslant \bar{h}(\tilde{x})-|\tilde{x}-\tilde{y}|-1 \\
& =h(\tilde{y})-1
\end{aligned}
$$

and likewise $h(\tilde{y}) \geqslant h(y)-1$.

For every $x \in S$, the map $y \mapsto \bar{h}(x)+|x-y|_{1}$ preserves parity (recall the assumption that $\bar{h}$ preserves parity), and therefore so does $h$. So $h$ is a parity-preserving map such that $|h(y)-h(\tilde{y})| \leqslant 1$ whenever $y$ and $\tilde{y}$ are neighbors. This proves that $h$ is a graph homomorphism.

Now, we describe further how to prove the central theorem of this article, i.e. the profile theorem in the special case of piecewise affine height functions. We derive the desired asymptotic equality by showing two inequalities. One direction of the inequality arises by overcounting the number of height functions that are close to the piecewise affine height profile; the opposite direction arises by undercounting the same set. In both directions, we subdivide the region into small blocks, so that we can compare the entropy on each block to the local surface tension (see Definition 12 and Figure 10). To overcount, we consider all choices of boundary values on the boundaries of the blocks, and for each boundary value function we count all possible extensions into the interior of the blocks. To undercount we have to use much smaller blocks, with boundary values fixed to match the desired affine function exactly (after rescaling, and up to rounding). The details of the proof are given in Section 5. The more difficult part of the proof is the overcounting 
argument, which relies on robustness of the microscopic entropy. We expect this to be a major source of difficulty when adapting our methods to other models.

As one can see, the framework of this argument is quite general and it can be adapted to more complicated models and settings. For example, the model of graph homomorphism into the infinite $d$-regular tree, studied by some of the current authors in [MT20], is amenable to this approach. Additionally, the authors have applied the current strategy to $\mathbb{Z}$-valued homomorphisms sampled according to a random environment. This means that the underlying combinatorial model is the same as in the current article, but in the definition of the microscopic entropy, the (uniform) counting measure on $M\left(R_{n}, h_{\partial R_{n}}\right)$ is replaced by a randomly perturbed measure. The conclusion is a homogenized variational principle, meaning that the microscopic entropy $\operatorname{Ent}_{n}\left(M\left(R_{n}, h_{\partial R_{n}}\right)\right)$, now a random variable depending on the realization of the environment, converges in probability to the minimum of the macroscopic entropy, which is still a deterministic quantity. Furthermore, we hope the method applies to other height function models, such as domino tilings (as studied in e.g. [CKP01]), and perhaps even more general tilings (as in e.g. [She01, Thu90]).

\section{Microscopic entropy and surface tension}

In this section, we prove basic properties of the microscopic entropy and local surface tension. More precisely, we prove that ent $(s)$ is continuous (see Lemma 23), that $\operatorname{ent}_{n}(s) \rightarrow \operatorname{ent}(s)$ uniformly (see Lemma 24), and that Ent $_{R_{n}}$ is robust under small changes to boundary values (see Lemma 25).

All three of these proofs split into two cases: values of the slope $s$ that are close to 1 (that is, such that $|s|_{\infty} \geqslant 1-\varepsilon$ ), where there are comparatively few possible states because of the steep slope; and slopes away from 1 (i.e. $|s|_{\infty} \leqslant 1-\varepsilon$ ), where we can make arguments based on extending height functions from one domain to another via the Kirszbraun theorem (Theorem 19).

The first result we state is about the microscopic entropy for slopes close to 1 . This lemma is used in the remainder of the section to handle the case of $s$ close to 1 .

Lemma 21. Let $\delta>0$, let $s \in[-1,1]^{m}$ with $|s|_{\infty}>1-\delta$, and let $n \in \mathbb{N}$. Consider any boundary height function $h_{\partial Q_{n}} \in M\left(\partial Q_{n}\right)$ such that

$$
\sup _{z \in \partial Q_{n}}\left|h_{\partial Q_{n}}(z)-h_{\partial Q_{n}}^{s}(z)\right| \leqslant \delta n .
$$

Then,

$$
\operatorname{Ent}_{Q_{n}}\left(M\left(Q_{n}, h_{\partial Q_{n}}\right)\right)=\theta(\delta)+\theta_{\delta}\left(\frac{1}{n}\right) .
$$

Proof. First, consider the one-dimensional case, i.e. $m=1$. Then the problem reduces to a simple calculation. The main idea is that the large slope $s$ forces a height function $h_{Q_{n}} \in M\left(Q_{n}, h_{\partial Q_{n}}\right)$ to closely follow a line of slope \pm 1 . By counting the number of deviating edges we overestimate the number of height functions.

Indeed, we assume without loss of generality that $\mathbb{R}^{1} \ni s>1-\delta$ (the case $s<-(1-\delta)$ is symmetric). We want to count height functions $h_{Q_{n}} \in M\left(Q_{n}, h_{\partial Q_{n}}\right)$. The line graph 


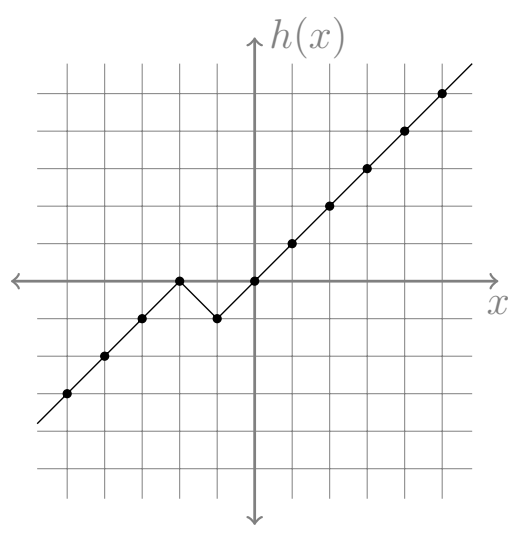

Figure 6: A one-dimensional height function with slope $s>1-\delta$. Because the slope is close to 1 , there cannot be many edges along which $h(x)$ decreases.

$Q_{n}=\{-n,-(n-1), \ldots, n-1, n\}$ has $2 n$ edges; let us write $k$ for the number of edges on which $h_{Q_{n}}$ decreases (see Figure 6). Then the height difference $h_{Q_{n}}(n)-h_{Q_{n}}(-n)$ is exactly $(2 n-k)-k$, which we simplify to $2(n-k)$. By $(20)$, we have (ignoring rounding errors)

$$
\begin{aligned}
h_{Q_{n}}(n)-h_{Q_{n}}(-n) & =h_{\partial Q_{n}}^{s}(n)-h_{\partial Q_{n}}^{s}(-n) \\
& =s n-s(-n) \\
& >2(1-\delta) n .
\end{aligned}
$$

Therefore, $2(n-k)>2(1-\delta) n$, so $k \leqslant \delta n$. It follows that

$$
\left|M\left(Q_{n}, h_{\partial Q_{n}}\right)\right| \leqslant\left(\begin{array}{c}
2 n \\
k
\end{array}\right) \leqslant\left(\begin{array}{c}
2 n \\
\lceil\delta n\rceil
\end{array}\right)
$$

and the limit

$$
\lim _{n \rightarrow \infty} \frac{1}{2 n+1} \log \left(\begin{array}{c}
2 n \\
\lceil\delta n\rceil
\end{array}\right)=-\delta \log \delta-(1-\delta) \log (1-\delta)=\theta(\delta)
$$

is an easy calculation using Stirling's formula.

For higher dimensions, we reduce to the one-dimensional case by treating the hypercube $\{-n, \ldots, n\}^{m}$ as the union of $(2 n+1)^{m-1}$ independent lines. In so doing we overestimate $\left|M\left(Q_{n}, h_{\partial Q_{n}}\right)\right|$, because we relax the graph homomorphism condition between lines. Thus

$$
\left|M\left(Q_{n}, h_{\partial Q_{n}}\right)\right| \leqslant\left(\begin{array}{c}
2 n \\
\lceil\delta n\rceil
\end{array}\right)^{(2 n+1)^{m-1}} .
$$

Taking a logarithm and dividing by $-\left|Q_{n}\right|=-(2 n+1)^{m}$ yields

$$
\operatorname{Ent}_{Q_{n}}\left(M\left(Q_{n}, h_{\partial Q_{n}}\right)\right) \geqslant-\frac{1}{2 n+1} \log \left(\begin{array}{c}
2 n \\
\lceil\delta n\rceil
\end{array}\right),
$$

which completes the proof (and in particular shows why the $\theta$ error terms do not depend on the dimension $m$ ). 
While Lemma 21 deals with slopes $s$ close to 1 , a different approach is needed for slopes away from 1. We use Theorem 19, which is a Kirszbraun theorem for graph homomorphisms. It gives a simple criterion for when a height function can be extended to larger domain. Lemma 22 applies the Kirszbraun theorem to derive entropy estimates. In particular, for two box sizes $n<\hat{n}$, the lemma compares $\operatorname{Ent}_{Q_{n}}\left(M\left(Q_{n}, h_{\partial Q_{n}}\right)\right)$ and $\operatorname{Ent}_{Q_{\hat{n}}}\left(M\left(Q_{\hat{n}}, h_{\partial Q_{\hat{n}}}\right)\right)$. The key idea is that any height function on the smaller box $Q_{n}$ can be extended to a height function on $Q_{\hat{n}}$, respecting the boundary data $h_{\partial Q_{\hat{n}}}$. Therefore (up to vanishing error terms), $\operatorname{Ent}_{Q_{\hat{n}}}\left(M\left(Q_{\hat{n}}, h_{\partial Q_{\hat{n}}}\right)\right) \leqslant \operatorname{Ent}_{Q_{n}}\left(M\left(Q_{n}, h_{\partial Q_{n}}\right)\right.$ ).

The extension requires that the boundary data $h_{\partial Q_{n}}$ and $h_{\partial Q_{\hat{n}}}$ be sufficiently similar. In particular, we will assume that both boundary height functions are close to linear height functions, with slopes $s$ and $\hat{s}$ respectively. The parameter $\varepsilon$ quantifies how close $h_{\partial Q_{n}}$ and $h_{\partial Q_{\hat{n}}}$ are to their respective linear height functions.

We also require that the slopes $s$ and $\hat{s}$ be close to each other, which is obviously necessary to apply the Kirszbraun theorem in our setting. Finally, we require that the two boxes sizes $n$ and $\hat{n}$ be not too different. In particular, we take $\hat{n}=(1+\delta) n$, where $\delta$ is a second approximation parameter. $\delta$ also shows up in a few other bounds, and in the conclusion of the lemma as a $\theta_{m}(\delta)$ error term.

This is not the simplest lemma of its kind that we could state, nor is it the most general. We choose to state these conditions because they are sufficient for our applications in this section. Moreover, they are necessary in the sense that simplifying any condition, e.g. by using only a single slope $s$ rather than two slopes, or by using linear boundary height functions without than allowing $\varepsilon$ fluctuations, would not suffice for our purposes.

Lemma 22. Let $\delta \in\left(0, \frac{1}{3}\right), \varepsilon \in\left(0, \frac{\delta^{2}}{2+\delta}\right], n, \hat{n} \in \mathbb{N}, s, \hat{s} \in[-1,1]^{m}, h_{\partial Q_{n}} \in M\left(\partial Q_{n}\right)$, and $h_{\partial Q_{\hat{n}}} \in M\left(\partial Q_{\hat{n}}\right)$ satisfy:

- $\hat{n}=\lceil(1+\delta) n\rceil$ or $\hat{n}=\lceil(1+\delta) n\rceil+1$,

- $|s|_{\infty} \leqslant 1-3 \delta$ and $|s-\hat{s}|_{\infty} \leqslant \frac{\delta^{2}}{1+\delta}$,

- $\max _{z \in \partial Q_{n}}\left|h_{\partial Q_{n}}(z)-s \cdot z\right| \leqslant \varepsilon n$, and

- $\max _{z \in \partial Q_{\hat{n}}}\left|h_{\partial Q_{\hat{n}}}(z)-\hat{s} \cdot z\right| \leqslant \varepsilon \hat{n}$.

See Figure 7 for a partial illustration. Then:

$$
\operatorname{Ent}_{Q_{\hat{n}}}\left(M\left(Q_{\hat{n}}, h_{\partial Q_{\hat{n}}}\right)\right) \leqslant \operatorname{Ent}_{Q_{n}}\left(M\left(Q_{n}, h_{\partial Q_{n}}\right)\right)+\theta_{m}(\delta)+\theta_{m}\left(\frac{1}{n}\right) .
$$

Proof of Lemma 22. We apply the Kirszbraun theorem (Theorem 19) to construct an injection from $M\left(Q_{n}, h_{\partial Q_{\hat{n}}}\right)$ into $M\left(Q_{\hat{n}}, h_{\partial Q_{\hat{n}}}\right)$. The existence of such an injection implies that

$$
\left|M\left(Q_{\hat{n}}, h_{\partial Q_{\hat{n}}}\right)\right| \geqslant\left|M\left(Q_{n}, h_{\partial Q_{n}}\right)\right|
$$

SO

$$
\operatorname{Ent}_{Q_{\hat{n}}}\left(M\left(Q_{\hat{n}}, h_{\partial Q_{\hat{n}}}\right)\right) \leqslant \frac{\left|Q_{n}\right|}{\left|Q_{\hat{n}}\right|} \operatorname{Ent}_{Q_{n}}\left(M\left(Q_{n}, h_{\partial Q_{n}}\right)\right) .
$$




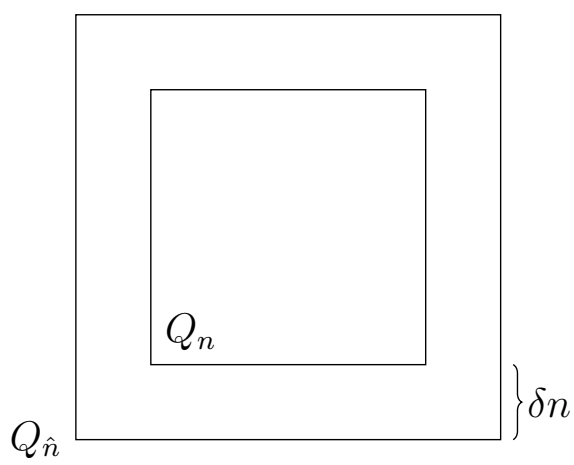

Figure 7: Domains for Lemma 22. The smaller domain $Q_{n}$ is a hypercube of side length $2 n+1$, and the larger domain $Q_{\hat{n}}$ has side length $2 \hat{n}+1$, where $\hat{n}=(1+\delta) n$.

Of course, $\frac{\left|Q_{n}\right|}{\left|Q_{\hat{n}}\right|}=\left(\frac{n}{\hat{n}}\right)^{m}+\theta_{m}\left(\frac{1}{n}\right)$ and

$$
\frac{n}{\hat{n}}=\frac{n}{(1+\delta) n}+\theta\left(\frac{1}{n}\right)=1+\theta(\delta)+\theta\left(\frac{1}{n}\right) .
$$

Since $\operatorname{Ent}_{Q_{n}}\left(M\left(Q_{n}, h_{\partial Q_{n}}\right)\right)$ is bounded (see Observation 11), the conclusion follows.

So, it remains to show that any height function $h_{Q_{n}} \in M\left(Q_{n}, h_{\partial Q_{n}}\right)$ can be extended to a height function in $M\left(Q_{\hat{n}}, h_{\partial Q_{\hat{n}}}\right)$. We want to apply the Kirszbraun theorem. The parity condition is automatic from our parity assumption in the definition of height functions (see Definition 1). We must verify inequality (18).

Let $z \in \partial Q_{n}$ and $\hat{z} \in \partial Q_{\hat{n}}$. By the triangle inequality,

$$
\begin{aligned}
\mid h_{\partial Q_{n}}(z) & -h_{\partial Q_{\hat{n}}}(\hat{z}) \mid \\
& \leqslant\left|h_{\partial Q_{n}}(z)-s \cdot z\right|+|s \cdot(z-\hat{z})|+|(s-\hat{s}) \cdot \hat{z}|+\left|\hat{s} \cdot \hat{z}-h_{\partial Q_{\hat{n}}}(\hat{z})\right| \\
& \leqslant \varepsilon n+|s|_{\infty}|z-\hat{z}|_{1}+|s-\hat{s}|_{\infty}+\varepsilon \hat{n} \\
& \leqslant \varepsilon n+(1-3 \delta)|z-\hat{z}|_{1}+\frac{\delta^{2}}{1+\delta}[(1+\delta) n+1]+\varepsilon[(1+\delta) n+1] \\
& =\varepsilon(2+\delta) n+(1-3 \delta)|z-\hat{z}|_{1}+\delta^{2} n+\frac{\delta^{2}}{1+\delta}+\varepsilon \\
& \leqslant \frac{\delta^{2}}{2+\delta}(2+\delta) n+(1-3 \delta)|z-\hat{z}|_{1}+\delta^{2} n+\frac{\delta^{2}}{1+\delta}+\frac{\delta^{2}}{2+\delta} \\
& \leqslant 2 \delta^{2} n+(1-3 \delta)|z-\hat{z}|_{1}+2 \delta^{2} .
\end{aligned}
$$

By definition, $|z-\hat{z}|_{1} \geqslant \hat{n}-n \geqslant \delta n$, so $2 \delta^{2} n \leqslant 2 \delta|z-\hat{z}|_{1}$. Moreover, the leftover term $2 \delta^{2}$ is $\leqslant 1$, so

$$
\left|h_{\partial Q_{n}}(z)-h_{\partial Q_{\hat{n}}}(\hat{z})\right| \leqslant(1-\delta)|z-\hat{z}|_{1}+1 .
$$

We assumed that $\delta|z-\hat{z}|_{1} \geqslant \delta^{2} n>1$, so the right-hand side above is strictly less that $|z-\hat{z}|_{1}$. Therefore the Kirszbraun theorem applies, which completes this proof.

Now, we may quickly state and prove a few useful properties of the microscopic entropy and surface tension, using Lemma 21 and Lemma 22 for the proofs. 
Lemma 23. The function $s \mapsto \operatorname{ent}(s)$, from $[-1,1]^{m}$ to $[-\log 2,0]$, is continuous. In fact, since the domain is compact, $s \mapsto \operatorname{ent}(s)$ is uniformly continuous.

Proof of Lemma 23. First, if $|s|_{\infty}=1$, Lemma $21 \operatorname{implies}$ ent $(s)=0$. As $|s|_{\infty} \rightarrow 1$, the same lemma implies ent $(s)=\theta\left(1-|s|_{\infty}\right) \rightarrow 0$. So, $s \mapsto \operatorname{ent}(s)$ is continuous at points $s$ with $|s|_{\infty}=1$.

Suppose instead that $|s|_{\infty}<1$. In the language of Lemma 22, let $\delta<\frac{1}{3} \wedge\left(1-|s|_{\infty}\right)$, let $\varepsilon=\frac{\delta^{2}}{2+\delta}$, and consider any $\hat{s}$ satisfying $|s-\hat{s}|_{\infty}<\frac{\delta^{2}}{1+\delta}$. For any $n, \hat{n}$ as in Lemma 22, take $h_{\partial Q_{n}}=h_{\partial Q_{n}}^{s}$ and $h_{\partial Q_{\hat{n}}}=h_{\partial Q_{\hat{n}}^{\hat{s}}}$. Then by Lemma 22,

$$
\operatorname{ent}_{n}(s) \geqslant \operatorname{ent}_{\hat{n}}(\hat{s})+\theta_{m}(\delta)+\theta_{m}\left(\frac{1}{n}\right) .
$$

Taking $n \rightarrow \infty$, we have ent $(s) \geqslant \operatorname{ent}(\hat{s})+\theta_{m}(\delta)$; taking $\delta \rightarrow 0$, we conclude that $\operatorname{ent}(s) \geqslant \liminf _{\hat{s} \rightarrow s} \operatorname{ent}(\hat{s})$. By exchanging the role of $s$ and $\hat{s}$, we conclude that $\operatorname{ent}(s) \leqslant$ $\lim \sup _{\hat{s} \rightarrow s} \operatorname{ent}(\hat{s})$. Therefore the function $s \mapsto \operatorname{ent}(s)$ is continuous.

Lemma 24. For a fixed dimension $m$, the convergence of $\operatorname{ent}_{n}(s) \rightarrow \operatorname{ent}(s)$ is uniform in $s \in[-1,1]^{m}$. In other words,

$$
\operatorname{ent}(s)=\operatorname{ent}_{n}(s)+\theta_{m}\left(\frac{1}{n}\right) .
$$

Proof of Lemma 24. Let $\varepsilon>0$. We want to find $n_{0}$, depending only on $\varepsilon$ and $m$, such that $n \geqslant n_{0}$ implies $\left|\operatorname{ent}_{n}(s)-\operatorname{ent}(s)\right|<\varepsilon$ for any $s \in[-1,1]^{m}$.

By Lemma 21 there exists $\delta>0$ such that $|s|_{\infty} \geqslant 1-\delta \operatorname{implies}\left|\operatorname{ent}_{n}(s)\right|<\varepsilon$ for all $n$. This suffices to handle the case $|s|_{\infty} \geqslant 1-\delta$.

For the remaining case of $|s|_{\infty} \leqslant 1-\delta$, we rely on compactness of the space $[-1+$ $\delta, 1-\delta]^{m}$. By uniform continuity of ent $(s)$ (see Lemma 22, there exists $\alpha>0$ such that $\left|s_{1}-s_{2}\right|_{\infty} \leqslant \alpha$ implies $\left|\operatorname{ent}\left(s_{1}\right)-\operatorname{ent}\left(s_{2}\right)\right|<\frac{\varepsilon}{4}$. Shrink $\delta$ if necessary so that $\frac{\delta^{2}}{1+\delta} \leqslant \alpha$, then shrink $\delta$ further so that the $\theta_{m}(\delta)$ term from (21), the conclusion of Lemma 22, is less than $\frac{\varepsilon}{4}$.

Choose a finite set of slopes $s^{(1)}, \ldots, s^{(k)} \in[-1+\delta, 1-\delta]^{m}$ such that for every $s^{*} \in$ $[-1+\delta, 1-\delta]^{m}$ there exists some $i=1, \ldots, k$ with $\left|s^{*}-s^{(i)}\right|_{\infty} \leqslant \frac{\delta^{2}}{1+\delta}$. Finally, choose $n_{0}$ large enough that whenever $n \geqslant \frac{1}{1-\delta} n_{0}$, the $\theta_{m}\left(\frac{1}{n}\right)$ term from (21) is less than $\frac{\varepsilon}{4}$, and for each $i=1, \ldots, k,\left|\operatorname{ent}_{n}\left(s^{(i)}\right)-\operatorname{ent}\left(s^{(i)}\right)\right|<\frac{\varepsilon}{4}$.

Let $n \geqslant n_{0}$, let $s^{*} \in[-1+\delta, 1-\delta]^{m}$ be arbitrary, and fix $i=1, \ldots, k$ such that $\left|s^{*}-s^{(i)}\right|_{\infty}<\frac{\delta^{2}}{1+\delta}$. We apply Lemma 22 twice. First take $s=s^{*}, \hat{s}=s^{(i)}, h_{\partial Q_{n}}=h_{\partial Q_{n}}^{s}$, and $h_{\partial Q_{\hat{n}}}=h_{\partial Q_{\hat{n}}}^{\hat{s}}$. The conclusion is

$$
\operatorname{ent}_{\hat{n}}\left(s^{(i)}\right) \leqslant \operatorname{ent}_{n}\left(s^{*}\right)+\theta_{m}(\delta)+\theta_{m}\left(\frac{1}{n}\right),
$$

and by our assumptions on $\delta$ and $n_{0}$ above, the $\theta$ terms simplify to

$$
\operatorname{ent}_{\hat{n}}\left(s^{(i)}\right) \leqslant \operatorname{ent}_{n}\left(s^{*}\right)+\frac{2 \varepsilon}{4}
$$


By choice of $n_{0}$,

and by choice of $s^{(i)}$,

$$
\operatorname{ent}_{\hat{n}}\left(s^{(i)}\right) \geqslant \operatorname{ent}\left(s^{(i)}\right)-\frac{\varepsilon}{4}
$$

$$
\operatorname{ent}\left(s^{(i)}\right) \geqslant \operatorname{ent}\left(s^{*}\right)-\frac{\varepsilon}{4}
$$

Combining the last three inequalities yields

$$
\operatorname{ent}_{n}(s) \geqslant \operatorname{ent}(s)-\varepsilon
$$

For the reverse inequality, choose $s=s^{(i)}, \hat{s}=s^{*}$, and exchange the role of $n$ and $\hat{n}$. Repeating the work above, we deduce the inequality

$$
\operatorname{ent}_{n}(s) \leqslant \operatorname{ent}(s)+\varepsilon
$$

which completes the proof of Lemma 24.

Lemma 25. Let $n \in \mathbb{N}, \varepsilon \in\left(0, \frac{1}{27}\right)$, and $s \in[-1,1]^{m}$. Let $h_{\partial Q_{n}} \in M\left(\partial Q_{n}\right)$ be such that

$$
\sup _{z \in \partial Q_{n}}\left|h_{\partial Q_{n}}(z)-h_{\partial Q_{n}}^{s}(z)\right| \leqslant \varepsilon n
$$

Then,

$$
\operatorname{Ent}_{Q_{n}}\left(M\left(Q_{n}, h_{\partial Q_{n}}\right)\right)=\operatorname{ent}(s)+\theta_{m}(\varepsilon)+\theta_{m, \varepsilon}\left(\frac{1}{n}\right)
$$

Proof. Suppose first that $|s|_{\infty} \geqslant 1-\varepsilon^{1 / 2}$. Then Lemma 21 applies to both $h_{\partial Q_{n}}$ and $h_{\partial Q_{n}}^{s}$, so

$$
\begin{aligned}
\operatorname{Ent}_{Q_{n}}\left(M\left(Q_{n}, h_{\partial Q_{n}}\right)\right) & =0+\theta(\varepsilon)+\theta_{\varepsilon}\left(\frac{1}{n}\right) \\
& =\operatorname{ent}_{n}(s)+\theta(\varepsilon)+\theta_{\varepsilon}\left(\frac{1}{n}\right) \\
& =\operatorname{ent}(s)+\theta_{m}(\varepsilon)+\theta_{m, \varepsilon}\left(\frac{1}{n}\right),
\end{aligned}
$$

where in the last line we used Lemma 24 for uniform convergence of $\operatorname{ent}_{n}(s) \rightarrow \operatorname{ent}(s)$, dependent only on dimension $m$.

So suppose instead that $|s|_{\infty} \leqslant 1-\varepsilon^{1 / 2}$. Apply Lemma 22 twice. Both times take $\hat{s}=s$ and $\delta=\sqrt{3 \varepsilon}$. Note that then $\varepsilon=\frac{\delta^{2}}{3} \leqslant \frac{\delta}{2+\delta}$, as required by Lemma 22. In the first application of Lemma 22 take $h_{\partial Q_{n}}=h_{\partial Q_{n}}$ and $h_{\partial Q_{\hat{n}}}=h_{\partial Q_{\hat{n}}}^{s}$ so that

$$
\operatorname{ent}_{\hat{n}}(s) \leqslant \operatorname{Ent}_{Q_{n}}\left(M\left(Q_{n}, h_{\partial Q_{n}}\right)\right)+\theta_{m}(\delta)+\theta_{m}\left(\frac{1}{n}\right) .
$$

The second time exchange $n$ and $\hat{n}$ to derive

$$
\operatorname{Ent}_{Q_{n}}\left(M\left(Q_{n}, h_{\partial Q_{n}}\right)\right) \leqslant \operatorname{ent}_{\hat{n}}(s)+\theta_{m}(\delta)+\theta_{m}\left(\frac{1}{n}\right)
$$

Since $\delta$ is determined by $\varepsilon$, we may replace $\delta$ by $\varepsilon$ in the $\theta$ terms above. And as

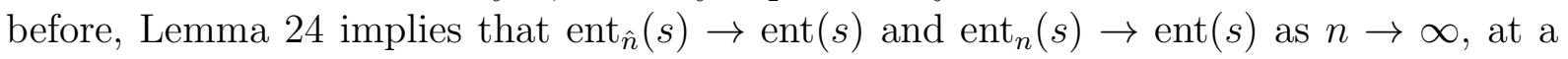
rate depending only on the dimension and on $\delta$ (since $n, \hat{n}$ differ from $n$ by a factor of $\left.(1+\delta)^{ \pm 1}\right)$. Therefore

$$
\operatorname{Ent}_{Q_{n}}\left(M\left(Q_{n}, h_{\partial Q_{n}}\right)\right)=\operatorname{ent}(s)+\theta_{m}(\varepsilon)+\theta_{m, \varepsilon}\left(\frac{1}{n}\right)
$$

as claimed. 


\section{Profile theorem for piecewise affine functions}

In this section we prove a simpler version of the profile theorem, restricted to the case where the domain $R$ is a finite union of simplices and where the asymptotic height function $h_{R}$ is piecewise affine, that is, affine when restricted to a single simplex. On one hand this case is simple enough that we can prove the profile theorem directly via over- and under-counting arguments (see the proof of Theorem 29 below). On the other hand, this case is sufficiently powerful to approximate general domains and height functions very well (see the proof of Theorem 15 and especially Lemma 31).

We must impose some regularity assumption on the simplices chosen; in particular we need the isoperimetric ratio to be bounded above (that is, the surface area of a simplex must not be too large in comparison to its volume). For simplicity we restrict our attention to certain families of simplices. Now let us introduce a standard notation describing these simplices.

In this exposition we follow [She05]. Given $w=\left(w_{1}, \ldots, w_{m}\right) \in \mathbb{R}^{m}$, we recall from the list of notations that $\lfloor w\rfloor:=\left(\left\lfloor w_{1}\right\rfloor, \ldots,\left\lfloor w_{m}\right\rfloor\right) \in \mathbb{Z}^{m}$. For a typical point $w \in \mathbb{R}$, let $\sigma(w)$ denote the permutation of $\{1, \ldots, m\}$ which rank-orders the components of $w-\lfloor w\rfloor$. In particular,

$$
w_{\sigma(1)}-\left\lfloor w_{\sigma(1)}\right\rfloor>w_{\sigma(2)}-\left\lfloor w_{\sigma(2)}\right\rfloor>\cdots>w_{\sigma(m)}-\left\lfloor w_{\sigma(m)}\right\rfloor .
$$

For example, consider the point $w=(1.1,-0.5,2.3) \in \mathbb{R}^{3}$. Then

$$
\lfloor w\rfloor=(1,-1,2) \quad \text { and } \quad w-\lfloor w\rfloor=(0.1,0.5,0.3) .
$$

Since the first largest coordinate in $w-\lfloor w\rfloor$ is at index 2, the second largest coordinate is at index 3 , and the third largest (i.e. the smallest) is at index 1 , we have $\sigma(w)=\left(\begin{array}{ll}2 & 31 \\ 1\end{array}\right)$.

Definition 26. Let $S_{m}$ denote the group of permutations on $\{1, \ldots, m\}$. For $v \in \mathbb{Z}^{m}$ and $\sigma \in S_{m}$, we define $C(v, \sigma)$ to be the closure of the set

$$
\left\{w \in \mathbb{R}^{m} \mid\lfloor w\rfloor=v, \sigma(w)=\sigma\right\} .
$$

A few members of the family $\left\{C(v, \sigma) \mid v \in \mathbb{Z}^{m}, \sigma \in S_{m}\right\}$ are illustrated in Figure 8 in the case of dimension $m=2$. It is an elementary observation that the $m$ ! simplices $\left\{C(0, \sigma) \mid \sigma \in S_{m}\right\}$ tile the hypercube $[0,1]^{m}$, i.e. $\bigcup_{\sigma \in S_{m}} C(0, \sigma)=[0,1]^{m}$, and any two simplices from $\left\{C(0, \sigma) \mid \sigma \in S_{m}\right\}$ only have at an $(m-1)$-dimensional intersection. It follows that, the infinite family $\left\{C(v, \sigma) \mid v \in \mathbb{Z}^{m}, \sigma \in S_{m}\right\}$ tiles $\mathbb{R}^{m}$.

Moreover, any two simplices $C\left(v_{1}, \sigma_{1}\right)$ and $C\left(v_{2}, \sigma_{2}\right)$ are isometric. That is, there exists a distance-preserving bijection $f: \mathbb{R}^{m} \rightarrow \mathbb{R}^{m}$ such that $f\left(C\left(v_{1}, \sigma_{1}\right)\right)=C\left(v_{2}, \sigma_{2}\right)$. This ensures that all the simplices $C(v, \sigma)$ have the same isoperimetric ratio. For our purposes we will also make reference to rescaled simplices.

Definition 27. For $\ell>0, v \in \mathbb{Z}^{m}$, and $\sigma \in S_{m}$, we write

$$
\ell C(v, \sigma):=\{\ell x \mid x \in C(v, \sigma)\}
$$

for scaled copy of the simplex $C(v, \sigma)$, scaled out from the origin. 


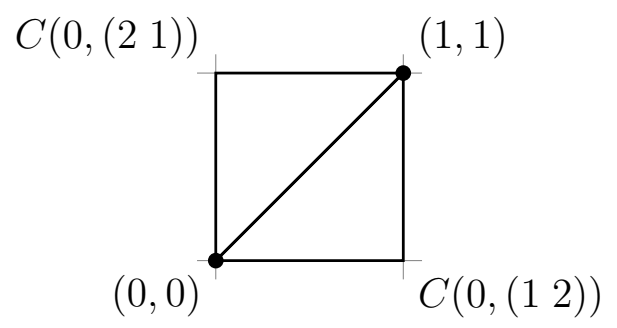

Figure 8: The two simplices in dimension 2 that tile the unit square. The simplex $C(0,(12))$ is the closure of the set of points $(x, y) \in[0,1]^{2}$ such that $x>y$, and $C(0,(21))$ is the closure of the points with $y>x$. The other simplices $\left\{C(v, \sigma) \mid v \in \mathbb{Z}^{m}, \sigma \in S_{2}\right\}$ are translates of these two simplices.

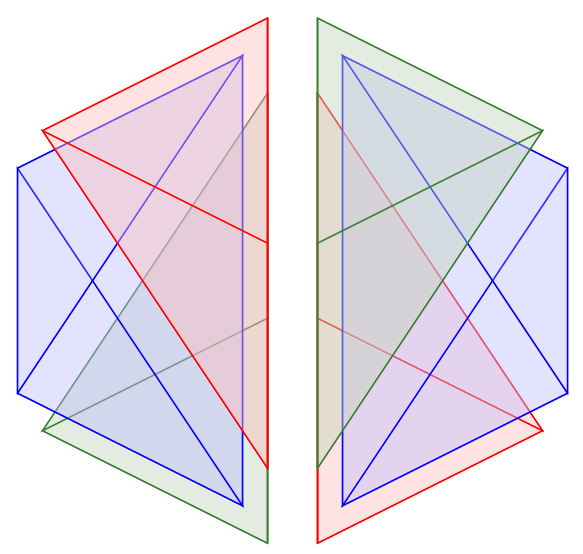

Figure 9: Decomposition of a unit cube into $\left\{C(0, \sigma) \mid \sigma \in S_{3}\right\}$. The simplices have been separated for a more clear figure.

As before, we observe that for any $\ell>0$, the collection of simplices $\{\ell C(0, \sigma) \mid \sigma \in$ $\left.S_{m}\right\}$ tiles the hypercube $[0, \ell]^{m}$. Therefore again, the family of translated simplices $\left\{\ell C(v, \sigma) \mid v \in \mathbb{Z}^{m}, \sigma \in S_{m}\right\}$ tiles $\mathbb{R}^{m}$. To approximate a general domain $R$ that satisfies Assumption 2, we consider domains which are the union of simplices.

Definition 28. For $\ell>0$, a simplex domain of scale $\ell$ is a region $K \subset \mathbb{R}^{m}$ that is the union of finitely many simplices of scale $\ell$. We further require that simplex domains be connected, so that a simplex domain $K$ automatically meets the requirements from Assumption 2.

For example, the union of the two simplices in Figure 8 is a simplex domain of scale 1. It is clear that simplex domains can approximate more general domains $R \subset \mathbb{R}^{m}$; we make this observation more precise in Lemma 31 below. Now, let us formulate the main result of this section, the simplicial profile theorem (Theorem 29). It is a special case of the profile theorem for simplex domains and piecewise affine height functions; cf. the general profile theorem (Theorem 15). 


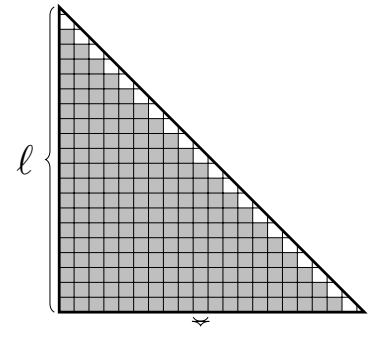

$q \approx \varepsilon \ell$

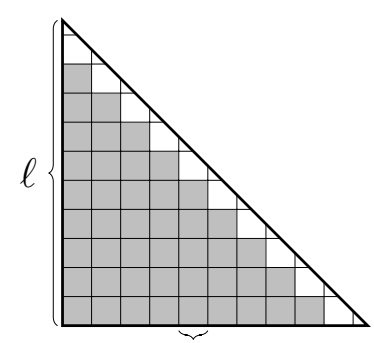

$q \approx \varepsilon^{1 / 2} \ell$

Figure 10: Decomposition of a single simplex into hypercubes at two scales. In both images, the shaded squares are the $Q_{i}$ from the proof of Theorem 29. The smaller squares on the left are used when undercounting the set $B\left(K_{n}, h_{K}, \varepsilon \ell\right)$ and the larger squares on the right are used when overcounting this set.

Theorem 29. Let $K=\Delta_{1} \cup \cdots \cup \Delta_{k}$ be a simplex domain of scale $\ell$, in the sense of Definition 28. Fix a height function $h_{K} \in M(K)$ such that each restriction $\left.h_{K}\right|_{\Delta_{j}}$, $j=1, \ldots, r$, is affine. Let $\varepsilon>0$, let $n \in \mathbb{N}$, and let $K_{n}:=\left\{z \in \mathbb{Z}^{m} \mid \frac{1}{n} z \in K\right\}$. Then for any slope $s \in[-1,1]^{m}$,

$$
\operatorname{Ent}_{K_{n}}\left(B\left(K_{n}, h_{K}, \varepsilon \ell\right)\right)=\frac{1}{|K|} \int_{K} \operatorname{ent}\left(\nabla h_{K}(x)\right) d x+\theta_{m}(\varepsilon)+\theta_{m, \varepsilon, \ell}\left(\frac{1}{n}\right) .
$$

Remark 30. In reading the proof of Theorem 29 for the first time, we encourage the reader to consider only a single simplex $\Delta$ rather than a simplex domain $K=\Delta_{1} \cup \cdots \cup \Delta_{k}$. The key ideas are more clear when thinking about a single simplex. In particular the simplex is decomposed into hypercubes two times, using hypercubes of a different scale each time. The two scales of hypercubes are illustrated in Figure 10. One decomposition is used to overestimate the microscopic entropy by undercounting the set $B\left(K_{n}, h_{K}, \varepsilon \ell\right)$. The other is used to underestimate the entropy by overcounting the set.

In the more general case of a simplex domain we still decompose twice, using hypercubes of a different size each time. A typical decomposition is illustrated in Figure 11. In particular we keep only those hypercubes that lie inside a single simplex, so that $h_{K}$ has a single, well-defined slope on each $Q_{i}$. Both sides of (22) are approximately additive over the simplices, but we will not explicitly prove this result here, nor do we rely on it.

Proof. As mentioned in Remark 30, we subdivide the region $K \subset \mathbb{R}^{m}$ into hypercubes $Q_{1}, Q_{2}, \ldots, Q_{r}$ of equal side length $q$. Two different values for the side length parameter $q$ are used at different times. The cubes $Q_{i}$ lie in a grid with their corners on the rescaled lattice $q \mathbb{Z}^{m} \subset \mathbb{R}^{m}$. The set $\left\{Q_{1}, \ldots, Q_{r}\right\}$ enumerates all such hypercubes that lie inside exactly one of the simplices $\Delta_{1}, \ldots, \Delta_{k}$, as illustrated in Figure 11. This ensures that $h_{K}$ has constant derivative on $Q_{i}$, which makes later arguments simpler. For $i=1, \ldots, r$ we choose $s_{i} \in[-1,1]^{m}$ and $b_{i} \in \mathbb{R}$ so that $\left.h_{K}\right|_{Q, i}=h_{Q_{i}}^{s_{i} \cdot x+b_{i}}$. Specifically, this means that

$$
s_{i}:=\nabla h_{K}\left(x_{i}\right) \quad \text { and } \quad b_{i}:=h_{K}\left(x_{i}\right)-s_{i} \cdot x_{i},
$$




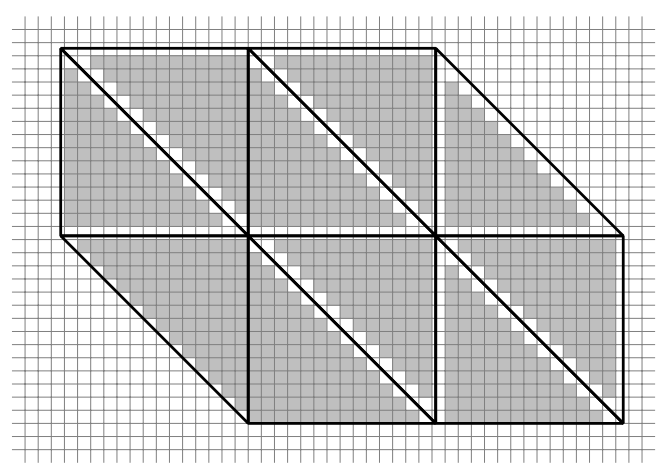

Figure 11: In Theorem 29, $K$ may be a simplex domain rather than a single simplex. Then only hypercubes that lie inside one of the simplices are part of the collection $\left\{Q_{i}\right\}$. These hypercubes are shaded. Note that there are still two different scales of hypercubes used, as illustrated in Figure 10, but only one scale is shown above. The set $G_{n}$ from later in the proof is the set of grid lines contained inside the simplex domain, and $U_{n}$ is the unshaded part of the simplex domain. $S_{n}$ is the union of $G_{n}$ and $U_{n}$.

for an arbitrarily chosen sample point $x_{i}$ from the interior of $Q_{i}$. The hypercubes $Q_{i}$ induce a decomposition of the discrete set $K_{n}$ into subsets $Q_{i, n}:=\left\{z \in \mathbb{Z}^{m} \mid \frac{1}{n} z \in Q_{i}\right\}$, plus a negligible remainder $K_{n} \backslash \bigcup_{i=1}^{r} Q_{i, n}$. This remainder is the unshaded part inside the triangles in Figure 11. We write $q n$ for the side length of the discrete hypercubes $Q_{i, n}$. Technically, each $Q_{i, n}$ has an integer side length $q_{i, n} \in \mathbb{Z}$ that is equal to either $\lfloor q n\rfloor$ or $\lceil q n\rceil$, but for simplicity we elide this detail in the rest of the proof.

Let us first sketch the main idea of the proof. We start with the integral on the right-hand side of (22). Since $h_{K}$ is piecewise affine, the integral reduces to a finite sum

$$
\begin{aligned}
\frac{1}{|K|} \int_{K} \operatorname{ent}\left(\nabla h_{K}(x)\right) d x & =\sum_{i=1}^{r} \frac{1}{|K|} \int_{Q_{i}} \operatorname{ent}\left(\left.\nabla h_{K}\right|_{Q_{i}}(x)\right) d x+\theta_{m}\left(\frac{q}{\ell}\right) \\
& =\sum_{i=1}^{r} \frac{\left|Q_{i}\right|}{|K|} \operatorname{ent}\left(s_{i}\right)+\theta_{m}\left(\frac{q}{\ell}\right) \\
& =\frac{1}{r} \sum_{i=1}^{r} \operatorname{ent}\left(s_{i}\right)+\theta_{m}\left(\frac{q}{\ell}\right),
\end{aligned}
$$

where we recall that $s_{i}=\nabla h_{K}\left(x_{i}\right)$ for $x_{i} \in Q_{i}$. Both of the two values for the hypercube side length parameter $q$ are chosen so that $\theta_{m}\left(\frac{q}{\ell}\right)=\theta_{m}(\varepsilon)$. The $\theta_{m}\left(\frac{q}{\ell}\right)$ errors arise from the uncovered region $K \backslash \bigcup_{i=1}^{r} Q_{i}$, i.e. the unshaded parts of the simplex domain in Figure 11. Indeed, one simply compares the measure $\left|\Delta_{j}\right|=\frac{1}{m !} \ell^{m}$ against that of the smaller simplex $\Delta_{j}^{\prime}$ with sides moved $\sqrt{m} q$ units inwards. Any hypercube $Q_{i}$ that intersects $\Delta_{j}^{\prime}$ must lie inside $\Delta_{j}$. The $\theta_{m}\left(\frac{q}{\ell}\right)$ error bound follows.

Now, we turn to the left-hand side of (22). Our goal is to relate $\operatorname{Ent}_{K_{n}}\left(B\left(K_{n}, h_{K}, \varepsilon \ell\right)\right)$ to the sum at the right-hand side of (23). Towards this end, we will under- and over-count 
the set of height functions $B\left(K_{n}, h_{K}, \varepsilon \ell\right)$, in order to derive the over- and under-estimates

$$
\operatorname{Ent}_{K_{n}}\left(B\left(K_{n}, h_{K}, \varepsilon \ell\right)\right) \leqslant \frac{1}{r} \sum_{i=1}^{r} \operatorname{ent}\left(s_{i}\right)+\theta_{m}(\varepsilon)+\theta_{m, \varepsilon, \ell}\left(\frac{1}{n}\right)
$$

and

$$
\operatorname{Ent}_{K_{n}}\left(B\left(K_{n}, h_{K}, \varepsilon \ell\right)\right) \geqslant \frac{1}{r} \sum_{i=1}^{r} \operatorname{ent}\left(s_{i}\right)+\theta_{m}(\varepsilon)+\theta_{m, \varepsilon, \ell}\left(\frac{1}{n}\right) .
$$

Equations (23), (24), and (25), together with the observation made above that $\theta_{m}\left(\frac{q}{\ell}\right)=$ $\theta_{m}(\varepsilon)$, suffice to prove the theorem. In order to prove (24), we will undercount height functions in $B\left(K_{n}, h_{K}, \varepsilon \ell\right)$. We choose $q \approx \varepsilon \ell$ and consider only height functions that agree with $h_{K_{n}}$ on each boundary $\partial Q_{i, n}$. These boundary data, together with the small size of $Q_{i, n}$, ensure that $h_{K_{n}}$ satisfies the $\ell^{\infty}$ condition for membership in $B\left(K_{n}, h_{K}, \varepsilon \ell\right)$. Then, to prove (25), we overcount height functions. We choose a larger value $q \approx \varepsilon^{1 / 2} \ell$. Any height function $h_{n} \in B\left(K_{n}, h_{K}, \varepsilon \ell\right)$, when restricted to one of the boundary sets $\partial Q_{i, n}$ and rescaled appropriately, fluctuates away from $h_{K}$ by at most $\varepsilon \ell n=\varepsilon^{1 / 2} q n$. When $\varepsilon$ is small, this allows us to compare the entropy on $Q_{i}$ to $\operatorname{Ent}\left(M\left(Q_{i, n}, h_{\partial Q_{i, n}}^{s_{i}}\right)\right)=\operatorname{ent}_{q n}\left(s_{i}\right)$, where $s_{i}=\nabla h_{K}\left(x_{i}\right)$ for $x_{i} \in Q_{i}$.

Now, let us describe the undercounting argument in detail. We seek to derive (24), an overestimate of $\operatorname{Ent}_{K_{n}}\left(B\left(K_{n}, h_{K}, \varepsilon \ell\right)\right)$, by undercounting the set $B\left(K_{n}, h_{K}, \varepsilon \ell\right)$. We take the side length of the hypercubes $\left\{Q_{i}\right\}$ to be $q=\frac{1}{4} \varepsilon \ell$.

We define an injection from the Cartesian product $\prod_{i} M\left(Q_{i, n}, h_{\partial Q_{i, n}}^{s_{i} \cdot x+b_{i}}\right)$ into the ball $B\left(K_{n}, h_{K}, \varepsilon \ell\right)$ in the natural way: given a tuple of height functions $h_{Q_{i, n}} \in M\left(Q_{i, n}, h_{\partial Q_{i, n}}^{s_{i} \cdot x+b_{i}}\right)$ for $i=1, \ldots, r$, we define $h_{K_{n}}(z):=h_{Q_{i, n}}(z)$ if $z \in Q_{i, n}$. For $z \in K_{n} \backslash \bigcup_{i=1}^{r} Q_{i, n}$, if $\frac{1}{n} z \in \Delta_{i}$ we set $h_{K_{n}}(z)=h_{\{z\}}^{s_{i} \cdot x+b_{i}}(z)$. It follows from Lemma 8 that this function $h_{K_{n}}$ is a height function,

Let us check that $h_{K_{n}} \in B\left(K_{n}, h_{K}, \varepsilon \ell\right)$. For $z \in K_{n} \backslash \bigcup_{i=1}^{r} Q_{i, n}$, the estimate $\mid \frac{1}{n} h_{K_{n}}(z)-$ $h_{K}\left(\frac{1}{n} z\right) \mid \leqslant \varepsilon \ell$ is immediate from the definition of $h_{K_{n}}(z)$. So, suppose that $z \in Q_{i, n}$ for some $i=1, \ldots, r$, and let $z^{\prime} \in Q_{i, n}$ be a boundary point in $\partial Q_{i, n}$ that minimizes $\ell^{1}$ distance from $z$. In particular, $\left|z-z^{\prime}\right|_{1} \leqslant q n$, so

$$
\begin{aligned}
\left|\frac{1}{n} h_{K_{n}}(z)-h_{K}\left(\frac{1}{n} z\right)\right| & =\left|\frac{1}{n} h_{Q_{i, n}}(z)-h_{Q_{i}}\left(\frac{1}{n} z\right)\right| \\
& \leqslant\left|\frac{1}{n} h_{Q_{i, n}}(z)-\frac{1}{n} h_{Q_{i, n}}\left(z^{\prime}\right)\right|+\left|\frac{1}{n} h_{Q_{i, n}}\left(z^{\prime}\right)-h_{Q_{i}}\left(\frac{1}{n} z^{\prime}\right)\right|+\left|h_{Q_{i}}\left(\frac{1}{n} z^{\prime}\right)-h_{Q_{i}}\left(\frac{1}{n} z\right)\right| \\
& \leqslant \frac{1}{n}\left|z-z^{\prime}\right|_{1}+\frac{1}{n}+\frac{1}{n}\left|z-z^{\prime}\right|_{1} \\
& \leqslant 3 q<\varepsilon \ell
\end{aligned}
$$

at least for $n$ large enough that $\frac{1}{n} \leqslant q=\frac{1}{4} \varepsilon \ell$. Therefore $h_{K_{n}} \in B\left(K_{n}, h_{K}, \varepsilon \ell\right)$ as desired. Thus

$$
\left|\prod_{i=1}^{r} M\left(Q_{i, n}, h_{\partial Q_{i, n}}^{s_{i} \cdot x+b_{i}}\right)\right| \leqslant\left|B\left(K_{n}, h_{K}, \varepsilon \ell\right)\right|,
$$




$$
\sum_{i=1}^{r} \frac{\left|Q_{i, n}\right|}{\left|K_{n}\right|} \operatorname{Ent}_{Q_{i, n}}\left(M\left(Q_{i, n}, h_{\partial Q_{i, n}}^{s_{i} \cdot x+b_{i}}\right)\right) \geqslant \operatorname{Ent}_{K_{n}}\left(B\left(K_{n}, h_{K}, \varepsilon \ell\right)\right)
$$

Now,

$$
\operatorname{Ent}_{Q_{i, n}}\left(M\left(Q_{i, n}, h_{\partial Q_{i, n}}^{s_{i} \cdot x+b_{i}}\right)\right)=\operatorname{ent}_{q n}\left(s_{i}\right)
$$

by translation invariance (see Observation 10). Moreover, because $q n=\frac{1}{4} \varepsilon \ell n$, we have $\operatorname{ent}_{q n}\left(s_{i}\right) \rightarrow \operatorname{ent}\left(s_{i}\right)$ as $n \rightarrow \infty$ at a rate dependent on $\varepsilon \ell$; by Lemma 24 , the convergence is uniform with respect to $s_{i}$. In other words, $\operatorname{ent}_{q n}\left(s_{i}\right)=\operatorname{ent}\left(s_{i}\right)+\theta_{m, \varepsilon, \ell}\left(\frac{1}{n}\right)$. Therefore, recalling (26), we have

$$
\operatorname{Ent}_{K n}\left(B\left(K_{n}, h_{K}, \varepsilon \ell\right)\right) \leqslant \sum_{i=1}^{r} \frac{\left|Q_{i, n}\right|}{\left|K_{n}\right|}\left(\operatorname{ent}\left(s_{i}\right)+\theta_{m, \varepsilon, \ell}\left(\frac{1}{n}\right)\right) .
$$

Now, the difference between $\sum_{i=1}^{r} \frac{\left|Q_{i, n}\right|}{\left|K_{n}\right|}$ and $\frac{1}{r}$ is $\theta_{m}(\varepsilon)+\theta_{m, \varepsilon, \ell}\left(\frac{1}{n}\right)$, where the first error term accounts for the unshaded part of Figure 11, and the second term is due to discretization effects. Therefore (27) simplifies to

$$
\operatorname{Ent}_{K_{n}}\left(B\left(K_{n}, h_{K}, \varepsilon \ell\right)\right) \leqslant \frac{1}{r} \sum_{i=1}^{r} \operatorname{ent}\left(s_{i}\right)+\theta_{m}(\varepsilon)+\theta_{m, \varepsilon, \ell}\left(\frac{1}{n}\right),
$$

which is exactly the overestimate $(24)$.

Now, we turn to (25). We will overcount $B\left(K_{n}, h_{K}, \varepsilon \ell\right)$ in order to underestimate the entropy $\operatorname{Ent}_{K_{n}}\left(B\left(K_{n}, h_{K}, \varepsilon \ell\right)\right)$. We will take side length $q$ of the hypercubes $Q_{i}$ to be $q=\varepsilon^{1 / 2} \ell$ for this part of the argument.

The basic idea is the following: we choose a subset $S_{n} \subset K_{n}$, and we only enforce the condition that $\left|\frac{1}{n} h_{K_{n}}(x)-h_{K}\left(\frac{1}{n} x\right)\right|<\varepsilon \ell$ from Definition 5 on $S_{n}$ rather than on all of $K_{n}$. $S_{n}$ is the complement of the (interiors of the) grid cells $Q_{i, n}$, so for any fixed height values on $S_{n}$, we can count the number of all extensions into the grid cells using a sum of entropy over the cells. There are many possible height values on $S_{n}$ that satisfy the $\varepsilon \ell$ error condition, but ultimately not too many because $S_{n}$ is small (compared to $K_{n}$ ).

Let us provide more detail. We define $S_{n}$ as follows. Let $G_{n}$ denote the grid formed by the boundaries of $Q_{i, n}$, i.e. the part of the grid lines from Figure 11 that lies inside the simplex domain. Let $U_{n}$ denote the points in $K_{n}$ that lie outside of any hypercube $Q_{i, n}$, i.e. the unshaded part of the simplex domain in Figure (11). Let $S_{n}:=G_{n} \cup U_{n}$. (As claimed, the complement $K_{n} \backslash S_{n}$ is the interior of the grid cells $Q_{i, n}$.) Additionally, let $\operatorname{Adm}\left(S_{n}\right)$ denote the set

$$
\operatorname{Adm}\left(S_{n}\right):=\left\{\text { "admissible" height functions on } S_{n}\right\},
$$

where "admissible" means those height functions $h_{S_{n}} \in M\left(S_{n}\right)$ that admit an extension to a height function in $B\left(K_{n}, h_{K}, \varepsilon \ell\right)$. 
We claim that there is an injection from $B\left(K_{n}, h_{K}, \varepsilon \ell\right)$ into

$$
\biguplus_{h_{S_{n}} \in \operatorname{Adm}\left(S_{n}\right)} \prod_{i=1}^{r} M\left(Q_{i, n},\left.h_{S_{n}}\right|_{\partial Q_{i, n}}\right),
$$

where " $\biguplus$ " denotes the disjoint union (so for distinct height functions $h_{S_{n}}$ and $\tilde{h}_{S_{n}}$ in $\operatorname{Adm}\left(S_{n}\right)$, the product sets $\prod_{i} M\left(Q_{i, n},\left.h_{S_{n}}\right|_{\partial Q_{i, n}}\right)$ and $\prod_{i} M\left(Q_{i, n},\left.\tilde{h}_{S_{n}}\right|_{\partial Q_{i, n}}\right)$ are considered disjoint inside the set from $(29))$.

Indeed, for any $h_{K_{n}} \in B\left(K_{n}, h_{K}, \varepsilon \ell\right)$, the function $h_{S_{n}}:=\left.h_{K_{n}}\right|_{S_{n}}$ is by definition in $\operatorname{Adm}\left(S_{n}\right)$, and $\left(\left.h_{K_{n}}\right|_{Q_{i, n}}\right)_{i=1}^{r}$ lies in the Cartesian product from the right-hand side of (29). To see that this map is an injection, suppose that $h_{K_{n}}$ and $\tilde{h}_{K_{n}}$ map to the same point. Then by definition of the (purported) injection, $\left.h_{K_{n}}\right|_{Q_{i, n}}=\left.\tilde{h}_{K_{n}}\right|_{Q_{i, n}}$ for each hypercube $Q_{i, n}$. Additionally, since the right-hand side of (29) is a disjoint union, we have $\left.h_{K_{n}}\right|_{S_{n}}=\left.\tilde{h}_{K_{n}}\right|_{S_{n}}$. Since $K_{n}=\bigcup_{i} Q_{i, n} \cup S_{n}$, the two height functions $h_{K_{n}}$ and $\tilde{h}_{K_{n}}$ are identical. Therefore, the map is an injection, and so

$$
\begin{aligned}
\left|B\left(K_{n}, h_{K}, \varepsilon \ell\right)\right| & \leqslant \sum_{h_{S_{n}} \in \operatorname{Adm}\left(S_{n}\right)} \prod_{i=1}^{r}\left|M\left(Q_{i, n}, h_{S_{n}} \mid \partial Q_{i, n}\right)\right| \\
& \leqslant\left|\operatorname{Adm}\left(S_{n}\right)\right| \max _{h_{S_{n}} \in \operatorname{Adm}\left(S_{n}\right)} \prod_{i=1}^{r}\left|M\left(Q_{i, n}, h_{S_{n}} \mid \partial Q_{i, n}\right)\right| .
\end{aligned}
$$

Taking logarithms and multiplying by $-\frac{1}{\left|K_{n}\right|}$, we see that

$$
\begin{aligned}
\operatorname{Ent}_{K_{n}}\left(B\left(K_{n}, h_{K}, \varepsilon \ell\right)\right) \\
\quad \geqslant \min _{h_{S_{n}} \in \operatorname{Adm}\left(S_{n}\right)} \sum_{i=1}^{r}-\frac{1}{\left|K_{n}\right|} \log \left|M\left(Q_{i, n}, h_{S_{n}} \mid \partial Q_{i, n}\right)\right|-\frac{\log \left|\operatorname{Adm}\left(S_{n}\right)\right|}{\left|K_{n}\right|} \\
\quad=\min _{h_{S_{n}} \in \operatorname{Adm}\left(S_{n}\right)} \sum_{i=1}^{r} \frac{\left|Q_{i, n}\right|}{\left|K_{n}\right|} \operatorname{Ent}_{Q_{i, n}}\left(M\left(Q_{i, n}, h_{S_{n}} \mid \partial Q_{i, n}\right)\right)-\frac{\log \left|\operatorname{Adm}\left(S_{n}\right)\right|}{\left|K_{n}\right|} .
\end{aligned}
$$

Now, we are almost done. We use three more asymptotic identities in the right-hand side of (30) to derive (25). First and simplest, since $\frac{\left|Q_{i, n}\right|}{\left|K_{n}\right|}=\frac{1}{r}+\theta_{m}(\varepsilon)+\theta_{m, \varepsilon, \ell}\left(\frac{1}{n}\right)$ and Ent $_{Q_{i, n}}$ is bounded (Observation 11), we replace $\frac{\left|Q_{i, n}\right|}{\left|K_{n}\right|}$ by $\frac{1}{r}$ in (30).

Second, we apply Lemma 25 to replace $\operatorname{Ent}_{Q_{i, n}}\left(M\left(Q_{i, n},\left.h_{S_{n}}\right|_{\partial Q_{i, n}}\right)\right)$ by $\operatorname{ent}\left(s_{i}\right)$. We fix a height function $h_{S_{n}}$ that achieves the minimum, then apply the lemma on each of the hypercubes $Q_{i, n}$. We recall that the hypercubes have side length $q n=\varepsilon^{1 / 2} \ell n$. Since $h_{S_{n}}$ is admissible and since $\left.h_{K}\right|_{Q_{i}}=h_{Q_{i}}^{s_{i} \cdot x+b_{i}}, h_{S_{n}}$ is approximately affine, i.e.

$$
\left|h_{S_{n}}(z)-h_{\partial Q_{i, n}}^{s_{i} \cdot x+b_{i}}(z)\right| \leqslant \varepsilon \ell n=\varepsilon^{1 / 2} q n .
$$

So, Lemma 25 applies and yields

$$
\begin{aligned}
\operatorname{Ent}_{Q_{i, n}}\left(M\left(Q_{i, n},\left.h_{S_{n}}\right|_{\partial Q_{i, n}}\right)\right) & =\operatorname{ent}\left(s_{i}\right)+\theta_{m}\left(\varepsilon^{1 / 2}\right)+\theta_{m, \varepsilon^{1 / 2}}\left(\frac{1}{\varepsilon^{1 / 2} \ell n}\right) \\
& =\operatorname{ent}\left(s_{i}\right)+\theta_{m}(\varepsilon)+\theta_{m, \varepsilon, \ell}\left(\frac{1}{n}\right),
\end{aligned}
$$


where the last line is just a matter of hiding the functions like $\varepsilon^{1 / 2}$ inside our $\theta$-notation. (See Section 2.4 for the definition of $\theta$-notation.)

Finally, we claim that $\frac{1}{\left|K_{n}\right|} \log \left|\operatorname{Adm}\left(S_{n}\right)\right|=\theta_{m}(\varepsilon)+\theta_{m, \varepsilon, \ell}\left(\frac{1}{n}\right)$, where we recall that the set $\operatorname{Adm}\left(S_{n}\right)$ was defined in (28). To see this, fix a base point $z_{0} \in S_{n}$. There are at most $(2 \varepsilon n+1)$ choices for $h_{S_{n}}\left(z_{0}\right)$, by definition of $B\left(K_{n}, h_{K}, \varepsilon \ell\right)$. Then, since $S_{n} \subset \mathbb{Z}^{m}$ is connected (in the sense of graph theory), there are less than $2^{\left|S_{n}\right|}$ ways to extend $h_{S_{n}}$ to the rest of $S_{n}$. So, we must estimate $\left|S_{n}\right|$. We recall that $S_{n}=G_{n} \cup U_{n}$, where $G_{n}$ is the grid and $U_{n}$ the unshaded region in Figure 11. Since $G_{n}$ grows like $n^{m-1}$ while $\left|K_{n}\right|$ grows like $n^{m}$, we have $\frac{\left|G_{n}\right|}{\left|K_{n}\right|}=\theta_{m, \varepsilon, \ell}\left(\frac{1}{n}\right)$. Next, the part of $K \subset \mathbb{R}^{m}$ that lies outside of any hypercube $Q_{i}$, that is, the unshaded part of the simplex domain in Figure 11, is a $\theta_{m}(\varepsilon)$ fraction of the total volume of $K$. Even with discretization errors, $\frac{\left|U_{n}\right|}{\left|K_{n}\right|}=\theta_{m}(\varepsilon)+\theta_{m, \varepsilon, \ell}\left(\frac{1}{n}\right)$. Altogether,

$$
\begin{aligned}
\frac{1}{\left|K_{n}\right|} \log \left|\operatorname{Adm}\left(S_{n}\right)\right| & \leqslant \frac{1}{\left|K_{n}\right|} \log \left((2 \varepsilon \ell n+1) 2^{\left|S_{n}\right|}\right) \\
& =\frac{\log (2 \varepsilon \ell n+1)}{\left|K_{n}\right|}+\frac{\log 2}{\left|K_{n}\right|}\left(\left|G_{n}\right|+\left|U_{n}\right|\right) \\
& =\theta_{m}(\varepsilon)+\theta_{m, \varepsilon, \ell}\left(\frac{1}{n}\right) .
\end{aligned}
$$

Applying the three asymptotic identities above in (30), we derive

$$
\operatorname{Ent}_{K_{n}}\left(B\left(K_{n}, h_{K}, \varepsilon \ell\right)\right) \geqslant \frac{1}{r} \sum_{i=1}^{r} \operatorname{ent}\left(s_{i}\right)+\theta_{m}(\varepsilon)+\theta_{m, \varepsilon, \ell}\left(\frac{1}{n}\right),
$$

which is exactly (25). This completes the proof.

\section{Proof of the profile theorem}

In this section we extend Theorem 29, the profile theorem for piecewise affine height functions on simplex domains, to general asymptotic height theorem on general domains (subject to Assumption 2).

The proof is an approximation argument, and we will need some auxiliary results. The most helpful is the simplicial Rademacher theorem, which states that Lipschitz functions are well-approximated by piecewise affine functions on a simplex domain. The other auxiliary results are about robustness of the microscopic and macroscopic entropies under changes in the domain and in the asymptotic height profile.

The simplicial Rademacher theorem is a general fact about Lipschitz functions. There is nothing particular to our setting, except for the use of our term "asymptotic height function" instead of "Lipschitz function." Related results include [Sch14], which extends Lemma 31 from Lipschitz functions to Sobolev functions, but weakens the approximation somewhat and is therefore not suitable for our purposes here. The statement of the simplicial Rademacher theorem is adapted from [CKP01, Lemma 2.2], and the proof is inspired by the proof there. 
Lemma 31 (Simplicial Rademacher theorem). Let $R \subseteq \mathbb{R}^{m}$ be a region satisfying Assumption 2, and let $h_{R} \in M\left(R, h_{\partial R}\right)$ be an asymptotic height function on $R$. For any $\varepsilon>0$ and any $\ell>0$ sufficiently small (depending on $\varepsilon$ ), we may choose a simplex domain $K=\Delta_{1} \cup \cdots \cup \Delta_{k} \subseteq R$ of scale $\ell$ (see Definition 28) and a piecewise affine asymptotic height function $h_{K}: K \rightarrow \mathbb{R}$ (that is, an asymptotic height function such that each restriction $\left.h_{K}\right|_{\Delta_{i}}: \Delta_{i} \rightarrow \mathbb{R}$ is affine) that satisfy the following properties:

(a) $|R \backslash K|<\varepsilon$, where $|\cdot|$ denotes the Lebesgue measure, and $\left.d_{H}(K, R)<\varepsilon\right)$, where $d_{H}$ denotes Hausdorff metric;

(b) $\max _{x \in K}\left|h_{K}(x)-h_{R}(x)\right|<\frac{1}{2} \varepsilon \ell$; and

(c) on at least $a(1-\varepsilon)$ fraction of the points in $K$ (by Lebesgue measure), the gradients $\nabla h_{K}(x)$ and $\nabla h_{R}(x)$ agree to within $\varepsilon ;$ more precisely, $\frac{1}{|K|} \mid\left\{x \in K|| \nabla h_{K}(x)-\right.$ $\left.\left.\nabla h_{R}(x)\right|_{2} \geqslant \varepsilon\right\} \mid<\varepsilon$.

Remark 32. We recall that the Rademacher theorem states that a Lipschitz function $h_{R}$ is differentiable almost everywhere. However $\nabla h_{R}$ may be poorly behaved. The Rademacher theorem gives no control over $\nabla h_{R}$, and the Lipschitz property only implies boundedness of the derivative, not regularity. The simplicial Rademacher theorem provides an approximation both to $h_{R}$ and to its derivative. Moreover the approximating function $h_{K}$ has a very simple derivative, despite the potential wildness of $\nabla h_{R}$. The cost is that $h_{K}$ only approximates $h_{R}$ well on a (large) portion of the domain rather than almost everywhere, but for our purposes this is a good trade-off.

In fact, it is not necessary that the function $h_{R}$ be Lipschitz. Almost everywhere differentiability is sufficient.

Before giving the proof of Lemma 31, we state and prove the following lemma about the standard simplices from Definition 26.

Lemma 33. Let $\Delta$ be any of the simplices $C(v, \sigma)$ for $v \in \mathbb{Z}^{m}$ and $\sigma \in S_{m}$. The $m+1$ vertices of $\Delta$ can be labelled $x^{(0)}, \ldots, x^{(m)}$ in such a way that, for each $i=1, \ldots, m$,

$$
x^{(i)}-x^{(i-1)}=e^{(\sigma(i))},
$$

where for $1 \leqslant j \leqslant m$, $e^{(j)}$ denotes the $j$-th standard basis vector (i.e., all entries of $e^{(j)}$ are 0 , except the $j$-th entry, which is 1 ).

Remark 34. We encourage the reader to keep Figure 9 in mind (or better, in sight) while reading this proof.

Proof. For simplicity, we assume without loss of generality that $v=0$. We use the permutation $\sigma$ to define a path between vertices of the simplex $C(0, \sigma)$ starting at $(0, \ldots, 0)$ and ending at $(1, \ldots, 1)$. To construct the path, first observe that

$$
C(0, \sigma)=\left\{x=\left(x_{1}, \ldots, x_{m}\right) \in[0,1]^{m} \mid x_{\sigma(i)} \geqslant x_{\sigma(j)} \text { for all } i<j\right\} .
$$


In other words, the $\sigma(1)$-th component of $x$ must be greater than the $\sigma(2)$-th, which is greater than or equal to the $\sigma(3)$-th, and so on. The path travels from $(0, \ldots, 0)$ along the $\sigma(1)$-th axis to $e_{\sigma(1)}$, then parallel to the $\sigma(2)$-th axis to $e_{\sigma(1)}+e_{\sigma(2)}$, and so on up to $\sum_{i=1}^{m} e_{i}=(1, \ldots, 1)$. Numbering the vertices of the path from $x^{(0)}$ to $x^{(m)}$ proves the lemma.

Now, we are ready for the proof of Lemma 31.

Proof of Lemma 31. Let $\ell>0$. We choose the simplex domain $K=\Delta_{1} \cup \cdots \cup \Delta_{k}$ such that $\left\{\Delta_{1}, \ldots, \Delta_{k}\right\}$ enumerates all simplices of scale $\ell$ (cf. Definition 27) that are contained in $R$. We define the asymptotic height function $h_{K} \in M(K)$ to agree with $h_{R}$ on the vertices of the simplices in $K$, and we extend $h_{K}$ into the rest of each $\Delta_{i}$ by linear interpolation. We will show that, once $\ell$ is small enough, properties (a), (b), and (c) from Lemma 31 all hold.

First we prove (a). The fact that $|K|$ tends to $|R|$ as $\ell \rightarrow 0$ is elementary measure theory, and we omit the proof.

Recall from (3) that we define the Hausdorff metric $d_{H}$ in terms of the $\ell^{1}$ metric on $\mathbb{R}^{m}$, for reasons explained in Remark 3. Therefore, for the second part of (a), it suffices to show that

$$
R \subset K+B_{\ell^{1}}(0, \varepsilon):=\left\{x+\left.y|x \in K,| y\right|_{1}<\varepsilon\right\} .
$$

We do this by constructing a subset $R^{\prime} \subset R$ such that $R \subset R^{\prime}+B_{\ell^{1}}(0, \varepsilon)$ and $R^{\prime}+$ $B_{\ell^{1}}(0, \alpha) \subset R$ for some $\alpha<\varepsilon$. The latter condition ensures that, for $\ell$ small enough, $R^{\prime} \subset K$. Indeed, the $\ell^{1}$-diameter of a simplex of scale $\ell$ is $\leqslant m \ell$ (i.e. $\operatorname{diam}_{1} \Delta:=$ $\max \left\{|x-y|_{1} \mid x, y \in \Delta\right\} \leqslant m \ell$ ), so as long as $\ell<\frac{\alpha}{m}$, every point $x \in R^{\prime}$ belongs to a simplex $\Delta_{i}$ of scale $\ell$ which is part of $K$. Therefore $R^{\prime} \subset K$, so $R \subset K+B_{\ell^{1}}(0, \varepsilon)$ as intended.

We proceed to construct $R^{\prime}$. Since $R$ is the closure of its interior, $R \subset \bigcup_{x \in R^{\circ}} B_{\ell^{1}}(x, \varepsilon)$. By compactness, choose $x_{1}, \ldots, x_{k}$ such that $R \subset B_{\ell^{1}}\left(x_{1}, \varepsilon\right) \cup \cdots \cup B_{\ell^{1}}\left(x_{k}, \varepsilon\right)$. For $i=$ $1, \ldots, k$, let

$$
\alpha_{i}:=\max \left\{\tilde{\alpha}>0 \mid B_{\ell^{1}}\left(x_{i}, \tilde{\alpha}\right) \subset R\right\}
$$

Since $x_{i} \in R^{\circ}$, each $\alpha_{i}>0$; since $R$ is compact, each $\alpha_{i}<\infty$. Let $\alpha=\min \left\{\alpha_{1}, \ldots, \alpha_{k}, \frac{\varepsilon}{2}\right\}$, and set

$$
R^{\prime}=\left\{x \in R^{\circ} \mid B_{\ell^{1}}(x, \alpha) \subset R\right\} .
$$

By construction, $R^{\prime}+B_{\ell^{1}}(0, \alpha) \subset R$, and since $x_{1}, \ldots, x_{k} \in R^{\prime}, R \subset R^{\prime}+B_{\ell^{1}}(0, \varepsilon)$. So $R^{\prime}$ satisfies the relations claimed above, which completes the proof of (a).

For later use we strengthen the volume estimate from (a). Choose $\ell$ smaller so that

$$
|R \backslash K|<\varepsilon^{\prime}:=\min \left\{\frac{\varepsilon}{2}, \frac{\varepsilon}{2|R|}, \frac{1}{2}\right\} .
$$

In particular, this implies that

$$
|R|<\frac{1}{1-\varepsilon^{\prime}}|K|
$$


Let us describe the key idea used to prove (b) and (c). We consider points $x$ where $h_{R}$ is differentiable, and indeed where $h_{R}$ is locally approximated well by its first-order Taylor polynomial. Once the simplices $\Delta_{i}$ are small enough and contain a "good" point $x$, the vertices all lie close to $x$, so we can use the Taylor polynomial to estimate the values of $h_{R}$ on the vertices. This yields the proof of (b) and (c).

To be more precise, we define a set $S_{\rho_{0}}$ of "good" points. Recall that the Lipschitz function $h_{R}$ is almost everywhere differentiable, by the Rademacher theorem. Consider any point $x \in R$ at which $\nabla h_{R}$ exists. Define the Taylor polynomial

$$
L_{x}(y):=h_{R}(x)+\nabla h_{R}(x) \cdot(y-x) .
$$

By the definition of differentiability,

$$
\lim _{y \rightarrow x} \frac{\left|h_{R}(y)-L_{x}(y)\right|}{|x-y|_{2}}=0
$$

so there exists $r_{0}(x)>0$ such that, for any $y \in R$ with $|y-x|_{2}<r_{0}(x)$,

$$
\left|h_{R}(y)-L_{x}(y)\right|<\left(\frac{\varepsilon}{4 \sqrt{m}} \wedge \frac{\varepsilon}{2 m}\right)|y-x|_{2} .
$$

(Recall that $m \in \mathbb{N}$ is the dimension parameter; we could replace the parenthesized expression by $\frac{\varepsilon}{4 m}$, but the expressions $\frac{\varepsilon}{4 \sqrt{m}}$ and $\frac{\varepsilon}{2 m}$ are useful later.) For $\rho>0$, define the set $S_{\rho} \subset R$ by

$$
S_{\rho}:=\left\{x \in R \mid r_{0}(x) \geqslant \rho\right\} .
$$

As $\rho \rightarrow 0$, the sets $S_{\rho}$ increase to the full-measure subset of $R$ on which $h_{R}$ is differentiable. Therefore $\left|S_{\rho}\right| \rightarrow|R|$ as $\rho \rightarrow 0$, and in particular, there exists $\rho_{0}>0$ such that

$$
\left|S_{\rho_{0}}\right| \geqslant\left(1-\frac{\varepsilon^{\prime}}{2}\right)|R|
$$

We choose $\ell_{0} \leqslant \frac{\rho_{0}}{\sqrt{m}}$. By the Pythagorean theorem (in $m$ dimensions), if $x, y$ are two points that lie in a simplex $\Delta_{i}$ and if $x \in S_{\rho_{0}}$, then $|x-y|_{2} \leqslant \sqrt{m} \ell<\rho_{0} \leqslant r(x)$. Therefore by $(33)$,

$$
\max _{y \in \Delta_{i}}\left|h_{R}(y)-L_{x}(y)\right|<\frac{\varepsilon \ell}{4} \wedge \frac{\varepsilon \ell}{2 \sqrt{m}} .
$$

There are two more steps to prove (b). First, under the assumption that $x \in \Delta_{i} \cap S_{\rho_{0}}$, we have compared $\left.h_{R}\right|_{\Delta_{i}}$ to the Taylor polynomial of $h_{R}$ centered at $x$; we should also compare $h_{K}$ to the same polynomial. Second, we show that at least $(1-\varepsilon) k$ of the simplices have some intersection with $S_{\rho_{0}}$. Then it is straightforward to complete the proof of (b).

Regarding $h_{K}$, recall that on the vertices $y_{0}, \ldots, y_{m}$ of $\Delta_{i}, h_{K}$ agrees with $h_{R}$. Therefore by (35),

$$
\max _{0 \leqslant i \leqslant m}\left|h_{K}\left(y_{i}\right)-L_{x}\left(y_{i}\right)\right|<\frac{\varepsilon \ell}{4}
$$


The function $y \mapsto h_{K}(y)-\left(h_{R}(x)+\nabla h_{R}(x) \cdot(y-x)\right)$ is affine, so $y \mapsto \mid h_{K}(y)-\left(h_{R}(x)+\right.$ $\left.\nabla h_{R}(x) \cdot(y-x)\right) \mid$ is convex. Hence

$$
\max _{y \in \Delta_{i}}\left|h_{K}(y)-L_{x}(y)\right|<\frac{\varepsilon \ell}{4} .
$$

Therefore, if a simplex $\Delta_{i}$ has any intersection with $S_{\rho_{0}}$, then $h_{K}$ satisfies the $L^{\infty}$ inequality from (b) over $\Delta_{i}$.

Now, let $k_{0}$ denote the number of simplices that intersect $S_{\rho_{0}}$. We claim that $k_{0} \geqslant$ $\left(1-\varepsilon^{\prime}\right) k$. Of course, since $S_{\rho_{0}}$ has large measure, it must intersect many of the simplices. More precisely, because $\left(k-k_{0}\right)$ is the number of simplices that do not intersect $S_{\rho_{0}}$,

$$
\left|S_{\rho_{0}}\right| \leqslant|R|-\left(k-k_{0}\right)\left|\Delta_{1}\right| \text {. }
$$

(Recall that the simplices of scale $\ell$ are isometric, so $\left|\Delta_{1}\right|=\cdots=\left|\Delta_{k}\right|=\frac{\ell^{m}}{m !}$.) Therefore

$$
\begin{array}{rlrl}
k-k_{0} & \leqslant \frac{|R|-\left|S_{\rho_{0}}\right|}{\left|\Delta_{1}\right|} & & (\text { By (34)) } \\
& \leqslant \frac{\varepsilon^{\prime}}{2} \frac{|R|}{\left|\Delta_{1}\right|} & & (\text { By (32)) } \\
& \leqslant \frac{\varepsilon^{\prime}}{2} \frac{\left(1-\varepsilon^{\prime}\right)^{-1}|K|}{\left|\Delta_{1}\right|} & & \text { (Since } \left.K=\bigcup_{i=1}^{k} \Delta_{i}\right) \\
& =\frac{\varepsilon^{\prime}}{2}\left(1-\varepsilon^{\prime}\right)^{-1} k & & \\
& \leqslant \varepsilon^{\prime} k, &
\end{array}
$$

since $\varepsilon^{\prime} \leqslant \frac{1}{2}$. So, both (35) and (36) apply on $k_{0} \geqslant\left(1-\varepsilon^{\prime}\right) k$ of the simplices. We throw away the "bad" simplices, at the cost of increasing $|R \backslash K|$ by at most

$$
\varepsilon^{\prime} k\left|\Delta_{i}\right| \leqslant\left(\frac{\varepsilon}{2|R|}\right) k\left(\frac{|R|}{k}\right)=\frac{\varepsilon}{2} .
$$

This is permissible by (31), since $|R \backslash K|$ was previously $\leqslant \frac{\varepsilon}{2}$. We have therefore proven (b).

For (c), we claim that if $x \in K \cap S_{\rho_{0}}$, then $\left|\nabla h_{R}(x)-\nabla h_{K}(x)\right|<\varepsilon$. By (32) and (34), $\left|K \cap S_{\rho}\right|>(1-\varepsilon)|R|$, so this will suffice to prove (c).

Let $x \in K \cap S_{\rho_{0}}$, and fix $i$ such that $x \in \Delta_{i}$. We will use Lemma 33 in order to make the calculations as concrete as possible. In particular, we label the vertices of $\Delta_{i}$ as $y_{0}, y_{1}, \ldots, y_{m}$ in such a way that $y_{i}-y_{i-1}=\ell e_{\sigma(i)}$, where $e_{\sigma(i)}$ is the $\sigma(i)$-th standard basis vector for some permutation $\sigma \in S_{m}$ of $\{1, \ldots, m\}$. Then by (35),

$$
\left|h_{R}\left(y_{i}\right)-L_{x}\left(y_{i}\right)\right|<\frac{\varepsilon \ell}{2 \sqrt{m}} \text { and }\left|h_{R}\left(y_{i-1}\right)-L_{x}\left(y_{i-1}\right)\right|<\frac{\varepsilon \ell}{2 \sqrt{m}},
$$


where we recall that $L_{x}(y)=h_{R}(x)+\nabla h_{R}(x) \cdot(y-x)$ is the first-order Taylor polynomial of $h_{R}$ at $x$. Combining these two inequalities,

$$
\left|\left(h_{R}\left(y_{i}\right)-h_{R}\left(y_{i-1}\right)\right)-\left(\nabla h_{R}(x) \cdot\left(y_{i}-y_{i-1}\right)\right)\right|<\frac{\varepsilon \ell}{\sqrt{m}} .
$$

Since $y_{i}-y_{i-1}=\ell e_{\sigma(i)}$,

$$
\left|\frac{h_{R}\left(y_{i}\right)-h_{R}\left(y_{i-1}\right)}{\left|y_{i}-y_{i-1}\right|_{2}}-\nabla h_{R}(x) \cdot \frac{y_{i}-y_{i-1}}{\left|y_{i}-y_{i-1}\right|_{2}}\right|<\frac{\varepsilon}{\sqrt{m}} .
$$

Because $h_{K}$ is the linear interpolation of $h_{R}$ from the vertices $y_{0}, \ldots, y_{m}$ to the rest of $\Delta_{i}$, we see that the first term on the left-hand side of (37) is

$$
\frac{h_{R}\left(y_{i}\right)-h_{R}\left(y_{i-1}\right)}{\left|y_{i}-y_{i-1}\right|_{2}}=\frac{h_{K}\left(y_{i}\right)-h_{K}\left(y_{i-1}\right)}{\left|y_{i}-y_{i-1}\right|_{2}}=\frac{\partial h_{K}}{\partial x_{\sigma(i)}}(x) .
$$

And of course, $\left(y_{i}-y_{i-1}\right) /\left|y_{i}-y_{i-1}\right|_{2}=e_{\sigma(i)}$, so the second term on the left-hand side of $(37)$ is

$$
\nabla h_{R}(x) \cdot \frac{y_{i}-y_{i-1}}{\left|y_{i}-y_{i-1}\right|_{2}}=\frac{\partial h_{R}}{\partial x_{\sigma(i)}}(x) .
$$

The last three equations hold for all $i=1, \ldots, m$. Therefore we may drop the permutation $\sigma(i)$ from the partial derivatives and conclude that, for every $i$,

$$
\left|\frac{\partial h_{K}}{\partial x_{i}}(x)-\frac{\partial h_{R}}{\partial x_{i}}(x)\right|<\frac{\varepsilon}{\sqrt{m}} .
$$

Thus

$$
\left|\nabla h_{K}(x)-\nabla h_{R}(x)\right|_{2}<\varepsilon
$$

The next three lemmas regard the robustness of the macroscopic entropy and microscopic entropy to changes in the domain and to changes in the limiting asymptotic height function. As seen in the simplicial Rademacher theorem (Lemma 31), we will change both the domain and the asymptotic height function. As long as these changes are small enough (in the appropriate senses), these lemmas show that the macroscopic entropy and microscopic entropy change by a small amount.

First, we deal with robustness of the macroscopic entropy. Because Ent $_{R}$ is an integral function with continuous and bounded integrand, robustness with respect to changes in both domain and asymptotic height function is easy to prove by standard analytic arguments. The main requirement is control over the change in the derivative of the asymptotic height function, as is provided by (c) from the simplicial Rademacher theorem (see Lemma 31).

Lemma 35. Let $\varepsilon>0$, and let $\tilde{R} \subseteq R \subset \mathbb{R}^{m}$ be sets meeting the assumptions from Assumption 2 with $|R \backslash \tilde{R}|<\varepsilon$. Let $h_{\tilde{R}} \in M(\tilde{R})$ and $h_{R} \in M(R)$ be such that

$$
\left|\left\{x \in \tilde{R}|| \nabla h_{\tilde{R}}(x)-\left.\nabla h_{R}(x)\right|_{2} \geqslant \varepsilon\right\}\right|<\varepsilon .
$$


Then,

$$
\operatorname{Ent}_{R}\left(h_{R}\right)=\operatorname{Ent}_{\tilde{R}}\left(h_{\tilde{R}}\right)+\theta_{m}(\varepsilon) .
$$

Proof. Recall: $\operatorname{Ent}_{R}\left(h_{R}\right)=\frac{1}{|R|} \int_{R} \operatorname{ent}\left(\nabla h_{R}(x)\right) d x$ and $\operatorname{Ent}_{\tilde{R}}\left(h_{\tilde{R}}\right)=\frac{1}{|\tilde{R}|} \int_{\tilde{R}} \operatorname{ent}\left(\nabla h_{\tilde{R}}(x)\right) d x$. Split $R$ into three parts.

The set $\left\{x \in \tilde{R}|| \nabla h_{\tilde{R}}(x)-\left.\nabla h_{R}(x)\right|_{2} \geqslant \varepsilon\right\}$ has measure less than $\varepsilon$ by hypothesis. Since ent $(s)$ is bounded (see Observation 13), the contribution of the points in this set to $\operatorname{Ent}_{\tilde{R}}\left(h_{\tilde{R}}\right)$ is within $\theta(\varepsilon)$ of the contribution to $\operatorname{Ent}_{R}\left(h_{R}\right)$.

Likewise, the set $R \backslash \tilde{R}$ has measure at most $\varepsilon$, so the contribution to $\operatorname{Ent}_{R}\left(h_{R}\right)$ is $\theta(\varepsilon)$. Of course, this set does not contribute to $\operatorname{Ent}_{\tilde{R}}\left(h_{\tilde{R}}\right)$.

Finally, for the remaining points $x,\left|\nabla h_{\tilde{R}}(x)-\nabla h_{R}(x)\right|_{2}<\varepsilon$. Since ent $(s)$ is uniformly continuous on its domain $s \in[-1,1]^{m}$, we have $\left|\operatorname{ent}\left(\nabla h_{\tilde{R}}(x)\right)-\operatorname{ent}\left(\nabla h_{R}(x)\right)\right|<\theta_{m}(\varepsilon)$. Since the integrands differ by at most $\theta_{m}(\varepsilon)$ and since the integrals are normalized by $\frac{1}{|R|}$ and $\frac{1}{|\widetilde{R}|}$, the contribution from this third part of the domain is also $\theta_{m}(\varepsilon)$.

Now, we turn to the microscopic entropy. Here it is easier to record two separate robustness results. The first is robustness with respect to changes in the asymptotic height function, and the second is robustness with respect to changes in domain. Robustness with respect to changes in the asymptotic height function comes immediately from the definition of the balls $B\left(R_{n}, h_{R}, \varepsilon\right)$.

Lemma 36. Let $\varepsilon>0$ and $n \in \mathbb{N}$. Let $R \subset \mathbb{R}^{m}$ satisfy Assumption 2 , and let $R_{n} \subset \mathbb{Z}^{m}$ satisfy $\frac{1}{n} R_{n} \subset R$. Let $h_{R}, \tilde{h}_{R} \in M(R)$ be two asymptotic height functions such that $\max _{x \in R}\left|h_{R}(x)-\tilde{h}_{R}(x)\right| \leqslant \varepsilon$. Then,

$$
\operatorname{Ent}_{R_{n}}\left(B\left(R_{n}, h_{R}, 2 \varepsilon\right)\right) \leqslant \operatorname{Ent}_{R_{n}}\left(B\left(R_{n}, \tilde{h}_{R}, \varepsilon\right)\right) .
$$

Proof. It suffices to notice that $B\left(R_{n}, h_{R}, 2 \varepsilon\right) \supseteq B\left(R_{n}, \tilde{h}_{R}, \varepsilon\right)$. This follows from the triangle inequality: for any $h_{R_{n}} \in B\left(R_{n}, \tilde{h}_{R}, \varepsilon\right)$ and any $z \in R_{n}$,

$$
\begin{aligned}
\left|\frac{1}{n} h_{R_{n}}(z)-h_{R}\left(\frac{1}{n} z\right)\right| & \leqslant\left|\frac{1}{n} h_{R_{n}}(z)-\tilde{h}_{R}\left(\frac{1}{n} z\right)\right|+\left|\tilde{h}_{R}\left(\frac{1}{n} z\right)-h_{R}\left(\frac{1}{n} z\right)\right| \\
& \leqslant \varepsilon+\varepsilon .
\end{aligned}
$$

More care is needed to state and prove robustness of the microscopic entropy with respect to changes in domain. The main idea is straightforward. Given two microscopic domains $\tilde{R}_{n} \subset R_{n}$, we will consider the extension map from $B\left(\tilde{R}_{n}, h_{R}, \varepsilon\right)$ to $B\left(R_{n}, h_{R}, \varepsilon\right)$ and the restriction map in the opposite direction. So long as every height function on the smaller domain admits an extension, we have $\left|B\left(\tilde{R}_{n}, h_{R}, \varepsilon\right)\right| \leqslant\left|B\left(R_{n}, h_{R}, \varepsilon\right)\right|$. In the opposite direction, the restriction map is not generally an injection but the pre-images are not too large; at most $2^{N}$ height functions on $R_{n}$ restrict to the any specific height function on $\tilde{h}_{R}$, where $N=\left|R_{n}\right| \backslash\left|\tilde{R}_{n}\right|$.

Most of the complications arise in the extension step. Our primary extension result, namely the Kirszbraun theorem (Theorem 19), is insufficient. It states that a height function $h_{\tilde{R}_{n}} \in B\left(\tilde{R}_{n}, h_{R}, \varepsilon\right)$ admits an extension to $R_{n}$, but that extension is not necessarily in 
$B\left(R_{n}, h_{R}, \varepsilon\right)$. There are two ways forward: to prove a stronger extension theorem specialized to the problem under consideration, or to leverage the Lipschitz property to control the extension. For greater generality, we prefer the second method. However there are a few difficulties: the Kirszbraun theorem is subtle when the asymptotic height profile has $\left|\nabla h_{K}\right|_{\infty}$ in part of the region, and the extension cannot generally be kept within distance $\varepsilon$ of $h_{R}$. This leads to the following somewhat complex formulation.

Lemma 37. Let $\varepsilon \in(0,1]$ and $n \in \mathbb{N}$ with $n \geqslant \frac{1}{\varepsilon}$. Let $\tilde{R} \subset R \subset \mathbb{R}^{m}$ and $\tilde{R}_{n} \subset R_{n} \subset \mathbb{Z}^{m}$ satisfy these assumptions:

$$
\begin{aligned}
\frac{1}{n} R_{n} & \subset R, & \frac{1}{n} \tilde{R}_{n} & \subset \tilde{R}, \\
d_{H}\left(\frac{1}{n} R_{n}, R\right) & =\theta(\varepsilon), & d_{H}\left(\frac{1}{n} \tilde{R}_{n}, \tilde{R}\right) & =\theta(\varepsilon),
\end{aligned}
$$

Additionally, assume that

$$
\frac{|R|}{|\tilde{R}|}=1+\theta(\varepsilon)
$$

Let $h_{R} \in M(R)$ be an asymptotic height function with $\operatorname{Lip}\left(h_{R}\right) \leqslant 1-c \varepsilon$ for some fixed $c \in(0,1]$. Then,

$$
\begin{aligned}
\operatorname{Ent}_{\tilde{R}_{n}}( & \left.B\left(\tilde{R}_{n}, h_{R}, \varepsilon\right)\right)+\theta(\varepsilon)+\theta_{\varepsilon}\left(\frac{1}{n}\right) \\
& \leqslant \operatorname{Ent}_{R_{n}}\left(B\left(R_{n}, h_{R}, \varepsilon\right)\right) \\
& \leqslant \operatorname{Ent}_{\tilde{R}_{n}}\left(B\left(\tilde{R}_{n}, h_{R}, \frac{c}{3} \varepsilon^{2}\right)\right)+\theta(\varepsilon)+\theta_{\varepsilon}\left(\frac{1}{n}\right) .
\end{aligned}
$$

Remark 38. The assumptions in Lemma 37 quantify the imprecise statements that $\tilde{R}$, $\frac{1}{n} R_{n}$, and $\frac{1}{n} R_{n}$ respectively approximate $R, R$, and $\tilde{R}$ from inside.

If we take the simplicial approximation $K$ from Lemma 31 to be $\tilde{R}$, and its discretization $K_{n}:=\left\{z \in \mathbb{Z}^{m} \mid \frac{1}{n} z \in K\right\}$ to be $\tilde{R}_{n}$, and if we recall the Assumption 2 about $R$ and $R_{n}$, then the hypotheses of Lemma 37 are satisfied, and moreover, we may replace all instance of $\theta(\varepsilon)$ in the conclusion by $\theta(\varepsilon \ell)$.

Proof. First, we prove the following inequalities:

$$
\left|B\left(R_{n}, h_{R}, \varepsilon\right)\right| \leqslant\left(2^{\left|R_{n}\right|}\right)^{\theta(\varepsilon)+\theta_{\varepsilon}\left(\frac{1}{n}\right)}\left|B\left(\tilde{R}_{n}, h_{R}, \varepsilon\right)\right|
$$

and

$$
\left|B\left(R_{n}, h_{R}, \varepsilon\right)\right| \geqslant\left|B\left(\tilde{R}_{n}, h_{R}, \frac{c}{3} \varepsilon^{2}\right)\right| .
$$

For (38), we note that every height function $h_{R_{n}} \in B\left(R_{n}, h_{R}, \varepsilon\right)$ restricts to a height function $\left.h_{R_{n}}\right|_{\tilde{R}_{n}} \in B\left(\tilde{R}_{n}, h_{R}, \varepsilon\right)$. Then, we claim that

$$
\left|R_{n}\right| n^{-d}=(1+\theta(\varepsilon))|R|
$$

and

$$
\left|\tilde{R}_{n}\right| n^{-d}=(1+\theta(\varepsilon))|\tilde{R}|
$$


To justify (40) we argue as follows. Consider the continuum region $R_{n}^{\square}:=\bigcup_{z \in R_{n}}\left(\left[0, \frac{1}{n}\right]^{d}+\right.$ $\left.\frac{1}{n} z\right)$, i.e. the union of hypercubes of side length $\frac{1}{n}$ translated by the points in $\frac{1}{n} R_{n}$. Clearly $R_{n}^{\square}$ has Lebesgue measure equal to $\left|R_{n}\right| n^{-d}$, and (like $\frac{1}{n} R_{n}$ ) satisfies $d_{H}\left(R_{n}^{\square}, R\right)=\theta(\varepsilon)$. This implies (40). Equation (41) is analogous. Further arithmetic yields the equation

$$
\frac{\left|R_{n}\right|}{\left|\tilde{R}_{n}\right|}=(1+\theta(\varepsilon))\left(1+\theta_{\varepsilon}\left(\frac{1}{n}\right)\right),
$$

and then

$$
\left|R_{n} \backslash \tilde{R}_{n}\right|=\left|R_{n}\right|\left(\theta(\varepsilon)+\theta_{\varepsilon}\left(\frac{1}{n}\right)\right) .
$$

Therefore the restriction map from $B\left(R_{n}, h_{R}, \varepsilon\right)$ to $B\left(\tilde{R}_{n}, h_{R}, \varepsilon\right)$ satisfies the following property: every height function $\tilde{h}_{\tilde{R}_{n}}$ in its image has at most $\left(2^{\left|R_{n}\right|}\right)^{\theta(\varepsilon)+\theta_{\varepsilon}\left(\frac{1}{n}\right)}$ pre-images; i.e. the restriction map is at most $\left(2^{\left|R_{n}\right|}\right)^{\theta(\varepsilon)+\theta_{\varepsilon}\left(\frac{1}{n}\right)}$-to-1. Inequality (38) follows immediately.

Now let us turn to (39). We want an injection from $B\left(\tilde{R}_{n}, h_{R}, \frac{c}{3} \varepsilon^{2}\right)$ into $B\left(R_{n}, h_{R}, \varepsilon\right)$. Fix a function $h_{\tilde{R}_{n}} \in B\left(\tilde{R}_{n}, h_{R}, \frac{c}{3} \varepsilon^{2}\right)$; we will construct an extension $h_{R_{n}} \in M\left(R_{n}\right)$.

Let

$$
R_{n}^{\prime}:=\left\{z \in R_{n} \mid d\left(z, \tilde{R}_{n}\right)>\frac{\varepsilon}{3} n\right\},
$$

where

$$
d\left(z, \tilde{R}_{n}\right):=\min _{\tilde{z} \in \tilde{R}_{n}}|z-\tilde{z}|_{1} .
$$

For $z \in R_{n}^{\prime}$, we arrange for the extension to satisfy $\left|h_{R_{n}}(z)-n h_{R}\left(\frac{1}{n} z\right)\right| \leqslant 1$. When $n h_{R}\left(\frac{1}{n} z\right)$ is not an integer, or is an integer but has the same parity as $z$, this inequality uniquely determines the value of $h_{R_{n}}(z)$. In the remaining case, there are two candidate values; we arbitrarily choose to "round down" to the lower value. Later it is important that we consistently round down (or up).

Let us check the hypotheses of the Kirszbraun theorem. If $\tilde{z} \in \tilde{R}_{n}$ and if $z \in R_{n}^{\prime}$, then $|\tilde{z}-z|_{1}>\frac{\varepsilon}{3} n$. Therefore

$$
\begin{aligned}
\mid h_{\tilde{R}_{n}}(\tilde{z}) & -h_{R_{n}}(z) \mid \\
& \leqslant\left|h_{\tilde{R}_{n}}(\tilde{z})-n h_{R}\left(\frac{1}{n} \tilde{z}\right)\right|+n\left|h_{R}\left(\frac{1}{n} \tilde{z}\right)-h_{R}\left(\frac{1}{n} z\right)\right|+\left|n h_{R}\left(\frac{1}{n} z\right)-h_{R_{n}}(z)\right| \\
& \leqslant \frac{c}{3} \varepsilon^{2} n+(1-c \varepsilon)|\tilde{z}-z|_{1}+1 \\
& <c \varepsilon|\tilde{z}-z|_{1}+(1-c \varepsilon)|\tilde{z}-z|_{1}+1 \\
& <|\tilde{z}-z|_{1}+1 \\
& \leqslant|\tilde{z}-z|_{1} .
\end{aligned}
$$

The argument for points $z_{1}, z_{2} \in R_{n}^{\prime}$ is similar to the arguments made in Section 2.2. By the triangle inequality, $\left|h_{R_{n}}\left(z_{1}\right)-h_{R_{n}}\left(z_{2}\right)\right| \leqslant\left|z_{1}-z_{2}\right|_{1}+2$. Equality holds only if both $n h_{R}\left(\frac{1}{n} z_{1}\right)$ and $n h_{R}\left(\frac{1}{n} z_{2}\right)$ are integers of the same parity as $z_{1}$ and $z_{2}$, respectively. In this case $h_{R_{n}}$ is rounded down at both points, so the Kirszbraun inequality is still satisfied. 
So, there exists an extension $h_{R_{n}}$ of $h_{\tilde{R}_{n}}$ such that $\left|h_{R_{n}}(z)-h_{R}\left(\frac{1}{n} z\right)\right| \leqslant 1$ for $z \in R_{n}^{\prime}$. We claim that $h_{R_{n}} \in B\left(R_{n}, h_{R}, \varepsilon\right)$. Since $\frac{c}{3} \varepsilon^{2} \leqslant \varepsilon$ and since $1 \leqslant \varepsilon n$, it suffices to consider points $z \in R_{n} \backslash R_{n}^{\prime}$. Fix such a $z$. By the definition of $R_{n}^{\prime}$, there exists $\tilde{z} \in \tilde{R}_{n}$ such that $|\tilde{z}-z| \leqslant \frac{\varepsilon}{3} n$. Note that $\frac{c}{3} \varepsilon^{2} \leqslant \frac{\varepsilon}{3}$, since $c, \varepsilon \leqslant 1$. By the Lipschitz property of $h_{R}$ and $h_{R_{n}}$,

$$
\begin{aligned}
h_{R_{n}}(z) & \leqslant h_{R_{n}}(\tilde{z})+\frac{\varepsilon}{3} n \\
& =h_{\tilde{R}_{n}}(\tilde{z})+\frac{\varepsilon}{3} n \\
& \leqslant n h_{R}\left(\frac{1}{n} \tilde{z}\right)+\frac{\varepsilon}{3} n+\frac{c}{3} \varepsilon^{2} n \\
& \leqslant n h_{R}\left(\frac{1}{n} \tilde{z}\right)+\frac{2 \varepsilon}{3} n \\
& \leqslant n h_{R}\left(\frac{1}{n} z\right)+\varepsilon n .
\end{aligned}
$$

By symmetry, $h_{R_{n}}(z) \geqslant n h_{R}\left(\frac{1}{n} z\right)-\varepsilon n$, and so $h_{R_{n}} \in B\left(R_{n}, h_{R}, \varepsilon\right)$. This extension process defines an injection from $B\left(\tilde{R}_{n}, h_{R}, \frac{c}{3} \varepsilon^{2}\right)$ into $B\left(R_{n}, h_{R}, \varepsilon\right)$, which proves (39).

Finally, we derive the conclusion from (38) and (39) by taking logarithms and normalizing, using (42) to account for the difference in normalizing factors $-\frac{1}{\left|R_{n}\right|}$ and $-\frac{1}{\left|\tilde{R}_{n}\right|}$.

Now, let us prove the profile theorem (Theorem 15). The main idea is straightforward: we approximate $h_{R}$ by a piecewise affine function (given by the simplicial Rademacher theorem, i.e. Lemma 31), for which we have already proven the simplicial profile theorem (Theorem 29). Then we use the robustness results (Lemma 35, Lemma 36, and Lemma 36) to deduce the profile theorem for $h_{R}$. However, in order to apply Lemma 36 we must first reduce to the case where the $\operatorname{Lipschitz}$ constant $\operatorname{Lip}\left(h_{R}\right):=\inf \left\{\lambda>0 \mid h_{R}\right.$ is $\lambda$-Lipschitz $\}$ is strictly less than 1 .

Proof of Theorem 15. First we reduce to the case where $\operatorname{Lip}\left(h_{R}\right) \leqslant 1-c \delta$, for a constant $c>0$ depending only on the domain $R$. Then, we reduce to the piecewise affine case of Theorem 29.

Reduction to $\operatorname{Lip}\left(\boldsymbol{h}_{\boldsymbol{R}}\right) \leqslant \mathbf{1}-\boldsymbol{c} \boldsymbol{\delta}$. By translation invariance, we may assume that there exists $x_{0} \in R$ with $h_{R}\left(x_{0}\right)=0$. Set $c=\frac{1}{2 \operatorname{diam}_{1} R} \wedge 1$, and define

$$
\tilde{h}_{R}:=(1-c \delta) h_{R}
$$

Then $\operatorname{Lip}\left(\tilde{h}_{R}\right) \leqslant 1-c \delta$, and for all $x \in R$, both $\left|h_{R}(x)-\tilde{h}_{R}(x)\right| \leqslant \frac{\delta}{2}$ and $\mid \nabla h_{R}(x)-$ $\nabla \tilde{h}_{R}(x) \mid \leqslant c \delta$. Assume that the conclusion holds for $\tilde{h}_{R}$, i.e.

$$
\operatorname{Ent}_{R}\left(\tilde{h}_{R}\right)=\operatorname{Ent}_{R_{n}}\left(B\left(R_{n}, \tilde{h}_{R}, \delta\right)\right)+\theta(\delta)+\theta_{\delta}\left(\frac{1}{n}\right) .
$$

We make two calculations. First,

$$
\begin{aligned}
\operatorname{Ent}_{R}\left(h_{R}\right) & \leqslant \operatorname{Ent}_{R}\left(\tilde{h}_{R}\right)+\theta(\delta) & & \text { (by Lemma 35) } \\
& \leqslant \operatorname{Ent}_{R_{n}}\left(B\left(R_{n}, \tilde{h}_{R}, 2 \delta\right)\right)+\theta(\delta)+\theta_{\delta}\left(\frac{1}{n}\right) & & \text { (by (43)) } \\
& \leqslant \operatorname{Ent}_{R_{n}}\left(B\left(R_{n}, h_{R}, \delta\right)\right)+\theta(\delta)+\theta_{\delta}\left(\frac{1}{n}\right) & & \text { (by Lemma 36). }
\end{aligned}
$$


Second,

$$
\begin{aligned}
\operatorname{Ent}_{R}\left(h_{R}\right) & \geqslant \operatorname{Ent}_{R}\left(\tilde{h}_{R}\right)+\theta(\delta) & & \text { (by Lemma 35) } \\
& \geqslant \operatorname{Ent}_{R_{n}}\left(B\left(R_{n}, \tilde{h}_{R}, \frac{1}{2} \delta\right)\right)+\theta(\delta)+\theta_{\delta}\left(\frac{1}{n}\right) & & \text { (by (43)) } \\
& \geqslant \operatorname{Ent}_{R_{n}}\left(B\left(R_{n}, h_{R}, \delta\right)\right)+\theta(\delta)+\theta_{\delta}\left(\frac{1}{n}\right) & & \text { (by Lemma 36). }
\end{aligned}
$$

So once we prove (43) with the extra hypothesis that $\operatorname{Lip}\left(\tilde{h}_{R}\right) \leqslant 1-c \delta$, the general result follows.

Reduction to piecewise linear height functions. We will apply Lemma 31 to derive a simplex domain $K$ and a piecewise linear height function $h_{K}$ approximating $R$ and $h_{R}$, then appeal to Theorem 29. In so doing we introduce two parameters: $\varepsilon$, which controls how well $K$ approximates $R$, and $\ell$, which controls the size of the simplices in $K$. There are a few important properties of $\varepsilon$ and $\ell$. First, $\delta=\varepsilon \ell$, so there is actually only one degree of freedom. Second, $\ell$ must be chosen to be sufficiently small, as is required by the simplicial Rademacher theorem (see Lemma 31). Third, as $\delta \rightarrow 0$ we must have $\varepsilon \rightarrow 0$, so that $\theta(\varepsilon)=\theta(\delta)$.

Let us describe explicitly how we choose $\varepsilon$ and $\ell$ satisfying these constraints. We fix a sequence $\varepsilon_{k} \downarrow 0$ (e.g. $\left.\varepsilon_{k}=\frac{1}{k}\right)$, and for each $k$ set

$$
\ell_{k}:=\frac{1}{2} \sup \left\{\ell>0 \mid \text { Lemma } 31 \text { applies with } \varepsilon=\varepsilon_{k}\right\} .
$$

We call attention to the fact that Lemma 31 is monotone in $\ell$. In particular, for any $\ell \leqslant \ell_{k}$, the conclusion of the lemma holds for $\left(\varepsilon_{k}, \ell\right)$. Now, let

$$
\delta_{k}:=\varepsilon_{k} \ell_{k} \wedge \frac{1}{2} \delta_{k-1} .
$$

The sequence $\delta_{k}$ decreases to 0 , so $\bigcup_{k=1}^{\infty}\left(\delta_{k+1}, \delta_{k}\right]$ is a non-trivial half-open interval with left endpoint at 0 . We assume that $\delta$ lies in this interval. Fix $k$ such that $\delta \in\left(\delta_{k+1}, \delta_{k}\right]$, and set $\varepsilon=\varepsilon_{k}$ and $\ell=\frac{\delta}{\varepsilon}$. Then $\delta=\varepsilon \ell$ by construction, and as noted above $\ell$ is small enough that the simplicial Rademacher theorem (see Lemma 31) applies. As $\delta \rightarrow 0$ necessarily $k \rightarrow \infty$, so $\varepsilon \rightarrow 0$ as desired. Therefore this choice of $\varepsilon$ and $\ell$ satisfies our criteria. Per Lemma 31, the corresponding simplex domain $K$ and piecewise affine asymptotic height function $h_{K}$ satisfy

$$
\begin{gathered}
|R \backslash K|<\varepsilon \quad \text { and } \quad d_{H}(K, R)<\varepsilon, \\
\max _{x \in K}\left|h_{K}(x)-h_{R}(x)\right|<\varepsilon \ell=\delta
\end{gathered}
$$

and

$$
\frac{1}{|K|}\left\{x \in K|| \nabla h_{K}(x)-\left.\nabla h_{R}(x)\right|_{2} \geqslant \varepsilon\right\}<\varepsilon .
$$

All that is left is to apply the simplicial profile theorem (see Theorem 29) and the 
robustness results (see Lemma 35, Lemma 36, and Lemma 36). In one direction, we have

$$
\begin{aligned}
\operatorname{Ent}_{R}\left(h_{R}\right) \leqslant & \operatorname{Ent}_{K}\left(h_{K}\right)+\theta(\varepsilon) & & \text { (Lemma 35 and }(46)) \\
\leqslant & \operatorname{Ent}_{K_{n}}\left(B\left(K_{n}, h_{K}, \varepsilon \ell\right)\right) & & \text { (Theorem 29) } \\
& +\theta(\varepsilon)+\theta_{\varepsilon, \ell}\left(\frac{1}{n}\right) & & \\
\leqslant & \operatorname{Ent}_{K_{n}}\left(B\left(K_{n}, h_{R}, \frac{1}{2} \varepsilon \ell\right)\right) & & \text { (Lemma 36 and }(45)) \\
& +\theta(\varepsilon)+\theta_{\varepsilon, \ell}\left(\frac{1}{n}\right) & & \\
\leqslant & \operatorname{Ent}_{R_{n}}\left(B\left(R_{n}, h_{R}, \frac{1}{2} \varepsilon \ell\right)\right) & & \text { (Lemma 37 and }(44)) \\
& +\theta(\varepsilon)+\theta_{\varepsilon, \ell}\left(\frac{1}{n}\right) & & \\
= & \operatorname{Ent}_{R_{n}}\left(B\left(R_{n}, h_{R}, \frac{1}{2} \delta\right)\right) & & \text { (choice of } \varepsilon \text { and } \ell) \\
& +\theta(\delta)+\theta_{\delta}\left(\frac{1}{n}\right) . & &
\end{aligned}
$$

By taking $\delta^{\prime}=\frac{1}{2} \delta$, this yields

$$
\operatorname{Ent}_{R}\left(h_{R}\right) \leqslant \operatorname{Ent} R_{n}\left(B\left(R_{n}, h_{R}, \delta^{\prime}\right)\right)+\theta\left(\delta^{\prime}\right)+\theta_{\delta^{\prime}}\left(\frac{1}{n}\right) .
$$

In the other direction,

$$
\begin{array}{rlrl}
\operatorname{Ent}_{R}\left(h_{R}\right) \geqslant & \operatorname{Ent}_{K}\left(h_{K}\right)+\theta(\varepsilon) & & \text { (Lemma 35 and (46)) } \\
\geqslant & \operatorname{Ent}_{K_{n}}\left(B\left(K_{n}, h_{K}, \varepsilon \ell\right)\right) & & \text { (Theorem 29) } \\
& +\theta(\varepsilon)+\theta_{\varepsilon, \ell}\left(\frac{1}{n}\right) & & \text { (Lemma 36 and (45)) } \\
\geqslant & \operatorname{Ent}_{K_{n}}\left(B\left(K_{n}, h_{R}, 2 \varepsilon \ell\right)\right) & \\
& +\theta(\varepsilon)+\theta_{\varepsilon, \ell}\left(\frac{1}{n}\right) & & \text { (Lemma 37 and }(44)) \\
\geqslant & \operatorname{Ent}_{R_{n}}\left(B\left(R_{n}, h_{R}, \frac{6}{c}(\varepsilon \ell)^{1 / 2}\right)\right) & \\
& +\theta(\varepsilon)+\theta_{\varepsilon, \ell}\left(\frac{1}{n}\right) & \\
= & \operatorname{Ent}_{R_{n}}\left(B\left(R_{n}, h_{R}, \frac{6}{c} \delta^{1 / 2}\right)\right) & & \text { (choice of } \varepsilon \text { and } \ell) \\
& +\theta(\delta)+\theta_{\delta}\left(\frac{1}{n}\right) . &
\end{array}
$$

By taking $\delta^{\prime \prime}=\frac{6}{c} \delta^{1 / 2}$, this yields

$$
\operatorname{Ent}_{R}\left(h_{R}\right) \leqslant \operatorname{Ent} R_{n}\left(B\left(R_{n}, h_{R}, \delta^{\prime \prime}\right)\right)+\theta\left(\delta^{\prime}\right)+\theta_{\delta^{\prime \prime}}\left(\frac{1}{n}\right) .
$$

Combining (47) and (48) completes the proof of the profile theorem.

\section{Proof of the variational principle}

Besides the profile theorem (Theorem 15), the proof of the variational principle (Theorem 16) relies on compactness of the space of asymptotic height functions. For robustness, we give a proof that does not assume that the macroscopic entropy functional admits a minimum. Note that the existence of such a minimizer is standard as soon as the local surface tension is convex and bounded below; see for example [CKP01, Section 2] or [She05]. 
However, for greater generality we work with the infimum of the macroscopic entropy and we do not assume that a minimizer exists. At any rate, it will be necessary to deal with infima (rather than minima) later when proving the large deviations principle.

Proof of Theorem 16. First, we shall prove that

$$
\inf _{h_{R} \in M\left(R, h_{\partial R}\right)} \operatorname{Ent}_{R}\left(h_{R}\right) \geqslant \operatorname{Ent}_{R_{n}}\left(M\left(R_{n}, h_{\partial R_{n}}, \delta\right)\right)+\theta(\delta)+\theta_{\delta}\left(\frac{1}{n}\right)
$$

via undercounting the number of height functions in $M\left(R_{n}, h_{\partial R_{n}}, \delta\right)$. The strategy is simple: we only count those height functions that are close to a "near-minimizer" of the macroscopic entropy. If we assume that a minimizer exists, i.e. that there exists $h_{R}^{\mathrm{min}} \in M\left(R, h_{\partial R}\right)$ such that

$$
\operatorname{Ent}_{R}\left(h_{R}^{\min }\right)=\inf _{h_{R} \in M\left(R, h_{\partial R}\right)} \operatorname{Ent}_{R}\left(h_{R}\right),
$$

then the following proof suffices. For any $\delta>0$ and $n \in \mathbb{N}$, the Definition 5 implies that

$$
B\left(R_{n}, h_{R}^{\min }, \delta\right) \subseteq M\left(R_{n}, h_{\partial R_{n}}, 2 \delta\right) .
$$

It follows immediately that

$$
\operatorname{Ent}_{R_{n}}\left(B\left(R_{n}, h_{R}^{\min }, \delta\right)\right) \geqslant \operatorname{Ent}_{R_{n}}\left(M\left(R_{n}, h_{\partial R_{n}}, 2 \delta\right)\right),
$$

so after applying the profile theorem and replacing $2 \delta$ by $\delta$,

$$
\operatorname{Ent}_{R_{n}}\left(M\left(R_{n}, h_{\partial R_{n}}, \delta\right)\right) \leqslant \inf _{h \in M\left(R, h_{\partial R}\right)} \operatorname{Ent}_{R}(h)+\theta(\delta)+\theta_{\delta}\left(\frac{1}{n}\right) .
$$

However, as mentioned above we want to give a proof that does not rely on the existence of a minimizer. This idea is also important for proving the large deviation principle below (see the paragraphs following (58) below). The first step is to replace $h_{R}^{\mathrm{min}}$ by a sequence of approximations, say $h_{R}^{(k)}$ satisfying

$$
\operatorname{Ent}_{R}\left(h_{R}^{(k)}\right) \leqslant \inf _{h_{R} \in M\left(R, h_{\partial R}\right)} \operatorname{Ent}\left(h_{R}\right)+\frac{1}{k} \quad \text { for } k \in \mathbb{N} .
$$

Also let $\theta^{(k)}(\delta)$ and $\theta_{\delta}^{(k)}\left(\frac{1}{n}\right)$ denote the $\theta$ terms from the profile theorem (Theorem 15) for the height function $h_{R}^{(k)}$. At this point one may be tempted to simply take the limit $k \rightarrow \infty$ for fixed $\delta$ and $n$. The problem is that the sequence $\theta^{(k)}(\delta)$ is not necessarily controlled as $k$ goes to infinity, and could in general diverge for any fixed $\delta>0$, and likewise for $\theta_{\delta}^{(k)}\left(\frac{1}{n}\right)$. To correct this, we proceed as follows.

Let $\delta_{0}=+\infty$ and $n_{0}=0$. For $k \geqslant 1$, choose $\delta_{k}$ such that

$$
0<\delta_{k} \leqslant \frac{1}{2} \delta_{k-1} \quad \text { and } \quad \theta^{(k)}\left(\delta_{k}\right) \leqslant \frac{1}{k}
$$


Now let $\delta>0$ and $n \in \mathbb{N}$ be given. Fix $k$ such that $\delta \in\left(\delta_{k}, \delta_{k-1}\right]$. Note that this is possible since $\delta_{k} \leqslant \frac{1}{2} \delta_{k-1}$ forces $\delta_{k} \rightarrow 0$ as $k \rightarrow \infty$. Since $\delta_{k}<\delta$, we have

$$
\operatorname{Ent}_{R_{n}}\left(B\left(R_{n}, h_{\partial R_{n}}, \delta\right)\right) \leqslant \operatorname{Ent}_{R_{n}}\left(B\left(R_{n}, h_{\partial R_{n}}, \delta_{k}\right)\right) .
$$

By the profile theorem (Theorem 15) applied to $h_{R}^{(k)}$,

$$
\operatorname{Ent}_{R_{n}}\left(B\left(R_{n}, h_{\partial R_{n}}, \delta_{k}\right)\right) \leqslant \operatorname{Ent}_{R}\left(h_{R}^{(k)}\right)+\theta^{(k)}\left(\delta_{k}\right)+\theta_{\delta_{k}}^{(k)}\left(\frac{1}{n}\right) .
$$

By choice of $h_{R}^{(k)}$, we have

$$
\operatorname{Ent}_{R}\left(h_{R}^{(k)}\right) \leqslant \inf _{h_{R} \in M\left(R, h_{\partial R}\right)} \operatorname{Ent}_{R}\left(h_{R}\right)+\frac{1}{k} .
$$

By choice of $\delta_{k}$,

$$
\theta^{(k)}\left(\delta_{k}\right) \leqslant \frac{1}{k}
$$

Finally, since $k$ and $\delta_{k}$ are determined from $\delta$,

$$
\theta_{\delta_{k}}^{(k)}\left(\frac{1}{n}\right)=\theta_{\delta}\left(\frac{1}{n}\right) \quad \text { and } \quad \frac{1}{k}=\theta(\delta)
$$

Putting it all together, we have

$$
\begin{aligned}
\operatorname{Ent}_{R_{n}}\left(B\left(R_{n}, h_{\partial R_{n}}, \delta\right)\right) & \leqslant \inf _{h_{R} \in M\left(R, h_{\partial R}\right)} \operatorname{Ent}_{R}\left(h_{R}\right)+\frac{2}{k}+\theta_{\delta_{k}}^{(k)}\left(\frac{1}{n}\right) \\
& =\inf _{h_{R} \in M\left(R, h_{\partial R}\right)} \operatorname{Ent}_{R}\left(h_{R}\right)+\theta(\delta)+\theta_{\delta}\left(\frac{1}{n}\right) .
\end{aligned}
$$

Now we prove the reverse inequality, namely

$$
\inf _{h_{R} \in M\left(R, h_{\partial R}\right)} \operatorname{Ent}_{R}\left(h_{R}\right) \leqslant \operatorname{Ent}_{R_{n}}\left(M\left(R_{n}, h_{\partial R_{n}}, \delta\right)\right)+\theta(\delta)+\theta_{\delta}\left(\frac{1}{n}\right) .
$$

Let $\varepsilon>0$. For each $h_{R} \in M\left(R, h_{\partial R}, 2 \delta\right)$, by the profile theorem (Theorem 15)

$$
\left|\operatorname{Ent}_{R_{n}}\left(B\left(R_{n}, h_{R}, \delta\right)\right)-\operatorname{Ent}_{R}\left(h_{R}\right)\right|<\theta(\delta)+\theta_{\delta}\left(\frac{1}{n}\right) .
$$

For each $h_{R} \in M\left(R, h_{\partial R}\right)$, fix $\eta\left(h_{R}\right)>0$ such that the $\theta(\delta)$ term in (51) satisfies

$$
\theta\left(\eta\left(h_{R}\right)\right) \leqslant \frac{\varepsilon}{2} .
$$

Recall from Definition 6 that

$$
M\left(R, h_{\partial R}, 2 \delta\right):=\left\{h_{R} \in \operatorname{Lip}(R)|\forall x \in \partial R,| h(x)-h_{\partial R}(x) \mid \leqslant 2 \delta\right\} .
$$


This set is compact as an easy consequence of the Arzelà-Ascoli theorem. Choose $h_{R}^{(1)} \in$ $M\left(R, h_{\partial R}\right)$ such that

$$
\operatorname{Ent}_{R}\left(h_{R}^{(1)}\right) \leqslant \inf _{h_{R} \in M\left(R, h_{\partial R}\right)} \operatorname{Ent}_{R}\left(h_{R}\right)+\varepsilon
$$

and pick $h_{R}^{(2)}, \ldots, h_{R}^{(k)}$ so that the union $\bigcup_{i=1}^{k} B\left(R, h_{R}^{(i)}, \eta\left(h_{R}^{(i)}\right)\right)$ covers $M\left(R, h_{\partial R}, 2 \delta\right)$, where $B\left(R, h_{R}^{(i)}, \eta\left(h_{R}^{(i)}\right)\right)$ is the set of asymptotic height functions $h_{R} \in M(R)$ that are uniformly within distance $\eta\left(h_{R}^{(i)}\right)$ of $h_{R}^{(i)}$. Note that the number $k$ of sets in this cover depends only on $\delta$. We abbreviate $\eta_{i}:=\eta\left(h_{R}^{(i)}\right)$. Moreover, we fix $n_{i} \in \mathbb{N}$ such that for all $n \geqslant n_{i}$, the $\theta_{\delta}\left(\frac{1}{n}\right)$ from (51) satisfies

$$
\theta_{\eta_{i}}\left(\frac{1}{n}\right) \leqslant \varepsilon
$$

The covering $M\left(R, h_{\partial R}, 2 \delta\right) \subseteq \bigcup_{i=1}^{k} B\left(R, h_{R}^{(i)}, \eta\left(h_{R}^{(i)}\right)\right)$ induces a covering of the set of height functions $M\left(R_{n}, h_{\partial R_{n}}, \delta\right)$. Indeed, consider an arbitrary height function $h_{R_{n}} \in$ $M\left(R_{n}, h_{\partial R_{n}}, \delta\right)$. After rescaling and interpolating (via the classical Kirszbraun theorem), we identify $h_{R_{n}}$ with a continuous function in $M\left(R, h_{\partial R}, 2 \delta\right)$. Under this identification,

$$
M\left(R_{n}, h_{\partial R_{n}}, \delta\right) \subseteq M\left(R, h_{\partial R}, 2 \delta\right) \subseteq \bigcup_{i=1}^{k} B\left(R, h_{R}^{(i)}, \eta_{i}\right) .
$$

This means that for any discrete height function $h_{R_{n}} \in M\left(R_{n}, h_{\partial R_{n}}, \delta\right)$ with continuous (rescaled) interpolation $\tilde{h}_{R_{n}} \in M\left(R, h_{\partial R}, 2 \delta\right)$ (note that $\delta$ increases to $2 \delta$ from discretization errors), there is $i \in\{1, \ldots, k\}$ such that $\sup _{x \in R}\left|\tilde{h}_{R_{n}}(x)-h_{R}^{(i)}(x)\right|<\eta_{i}$. By Definition 5, it follows that

$$
M\left(R_{n}, h_{\partial R_{n}}, \delta\right) \subseteq \bigcup_{i=1}^{k} B\left(R_{n}, h_{R}^{(i)}, \eta_{i}\right)
$$

Hence,

$$
\begin{aligned}
\operatorname{Ent}_{R_{n}}\left(M\left(R_{n}, h_{\partial R_{n}}, \delta\right)\right) & \geqslant \operatorname{Ent}_{R_{n}}\left(\bigcup_{i=1}^{k} B\left(R_{n}, h_{R}^{(i)}, \eta_{i}\right)\right) \\
& \geqslant-\frac{1}{\left|R_{n}\right|} \log \left(\sum_{i=1}^{k}\left|B\left(R_{n}, h_{R}^{(i)}, \eta_{i}\right)\right|\right) .
\end{aligned}
$$

Let us estimate $\left|B\left(R_{n}, h_{R}^{(i)}, \eta_{i}\right)\right|$. Assuming that $n$ is larger than all of the constants $n_{1}, \ldots, n_{k}$, then for each $i=1, \ldots, k$,

$$
\begin{array}{rlr}
\operatorname{Ent}_{R_{n}} & \left.B\left(R_{n}, h_{R}^{(i)}, \eta_{i}\right)\right) & \\
& \geqslant \operatorname{Ent}_{R}\left(h_{R}^{(i)}\right)-2 \varepsilon & \\
& \geqslant \inf _{h_{R} \in M\left(R, h_{\partial R}\right)} \operatorname{Ent}_{R}\left(h_{R}\right)-2 \varepsilon & \\
& \geqslant \operatorname{Ent}_{R}\left(h_{R}^{(1)}\right)-3 \varepsilon & \\
& \geqslant \operatorname{Ent}_{R_{n}}\left(B\left(R_{n}, h_{R}^{(1)}, \eta_{1}\right)\right)-5 \varepsilon & \text { (By (51), (52), and (53)). }
\end{array}
$$


In other words,

$$
\left|B\left(R_{n}, h_{R}^{(i)}, \eta_{i}\right)\right| \leqslant\left|B\left(R_{n}, h_{R}^{(1)}, \eta_{1}\right)\right| e^{5 \varepsilon\left|R_{n}\right|} .
$$

We apply this last estimate in (55) to derive

$$
\begin{aligned}
\operatorname{Ent}_{R_{n}}\left(M\left(R_{n}, h_{\partial R_{n}}, \delta\right)\right) & \geqslant-\frac{1}{\left|R_{n}\right|} \log \left(k\left|B\left(R_{n}, h_{R}^{(1)}, \eta_{1}\right)\right| e^{5 \varepsilon\left|R_{n}\right|}\right) \\
& =-\frac{1}{\left|R_{n}\right|} \log \left|B\left(R_{n}, h_{R}^{(1)}, \eta_{1}\right)\right|-\frac{\log k}{\left|R_{n}\right|}-5 \varepsilon \\
& =-\frac{1}{\left|R_{n}\right|} \log \left|B\left(R_{n}, h_{R}^{(1)}, \eta_{1}\right)\right|-\theta_{\delta}\left(\frac{1}{n}\right)-5 \varepsilon \\
& =-\inf _{h_{R} \in M\left(R, h_{\partial R}\right)} \operatorname{Ent}_{R}\left(h_{R}\right)-\theta_{\delta}\left(\frac{1}{n}\right)-6 \varepsilon .
\end{aligned}
$$

Here, note that since $k$ depends only on $\delta, \frac{k}{\left|R_{n}\right|}=\theta_{\delta}\left(\frac{1}{n}\right)$. Because $\varepsilon>0$ was arbitrary, this yields the desired estimate (50).

\section{Large deviations principle}

In this section we prove Theorem 17, the large deviations principle. For the reader's convenience, we recall the following definitions from the statement of the theorem in Section 2.5. For $\delta>0, n \in \mathbb{N}$, and $h_{R} \in M(R)$ :

$$
\begin{aligned}
\mu_{\delta, n} & :=\frac{1}{\left|M\left(R_{n}, h_{\partial R_{n}}, \delta\right)\right|}\left|\left\{h_{R_{n}} \in M\left(R_{n}, h_{\partial R_{n}}, \delta\right) \mid \tilde{h}_{R_{n}} \in A\right\}\right|, \\
r_{\delta, n} & :=\left|R_{n}\right|, \\
I\left(h_{R}\right) & := \begin{cases}\operatorname{Ent}_{R}\left(h_{R}\right)-E & \text { if }\left.h_{R}\right|_{\partial R}=h_{\partial R}, \\
\infty & \text { otherwise, }\end{cases}
\end{aligned}
$$

where $\tilde{h}_{R_{n}}$ is the piecewise-affine interpolation of the function $\frac{1}{n} z \mapsto \frac{1}{n} h_{R_{n}}(z)$ on the simplex domain with vertices $\left\{\frac{1}{n} K\right\}_{n \in R_{n}}$, and where $E=\inf _{h_{R} \in M\left(R, h_{\partial R}\right)} \operatorname{Ent}_{R}\left(h_{R}\right)$. The proof of the large deviations principle that we give here is based on the proof of the variational principle, Theorem 16, given in Section 7. We encourage the reader to read Section 7 first.

Proof of Theorem 17. First, we prove the LDP lower bound (16), i.e.

$$
-\inf _{h_{R} \in A^{\circ}} I\left(h_{R}\right) \leqslant \underline{\lim _{\delta \rightarrow 0}} \frac{\lim }{n \rightarrow \infty} \frac{1}{r_{\delta, n}} \log \mu_{\delta, n}(A) .
$$

Without loss of generality we may assume that $A$ is open. We may assume also that $\inf _{h_{R} \in A} I\left(h_{R}\right)<\infty$, or else (16) is trivial. By using these assumptions and replacing the symbols $\mu_{\delta, n}, r_{\delta, n}$, and $I\left(h_{R}\right)$ by their definitions, (16) simplifies to

$$
-\inf _{h_{R} \in A}\left(\operatorname{Ent}_{R}\left(h_{R}\right)-E\right) \leqslant \lim _{\delta \rightarrow 0} \lim _{n \rightarrow \infty} \frac{\left|\left\{h_{R_{n}} \in M\left(R_{n}, h_{\partial R_{n}}, \delta\right) \mid \tilde{h}_{R_{n}} \in A\right\}\right|}{\left|M\left(R_{n}, h_{\partial R_{n}}, \delta\right)\right|} .
$$


Simplifying further by multiplying by -1 and using our definition of the microscopic entropy (11), it suffices to prove

$$
\begin{aligned}
\inf _{h_{R} \in A} \operatorname{Ent}_{R}\left(h_{R}\right)-E \geqslant \varlimsup_{\delta \rightarrow 0} & \varlimsup_{n \rightarrow \infty} \operatorname{Ent}_{R_{n}}\left(\left\{h_{R_{n}} \in M\left(R_{n}, h_{\partial R_{n}}, \delta\right) \mid \tilde{h}_{R_{n}} \in A\right\}\right) \\
& -\varliminf_{\delta \rightarrow 0} \underline{\lim _{n \rightarrow \infty}} \operatorname{Ent}_{R_{n}}\left(M\left(R_{n}, h_{\partial R_{n}}, \delta\right)\right) .
\end{aligned}
$$

By the variational principle (Theorem 16),

$$
\varliminf_{\delta \rightarrow 0} \varliminf_{n \rightarrow \infty} \operatorname{Ent}_{R_{n}}\left(M\left(R_{n}, h_{\partial R_{n}}, \delta\right)\right)=E .
$$

After cancelling the corresponding terms in (57), and after replacing $\varlimsup$ im by our preferred $\theta$ asymptotics, it suffices to show that

$$
\inf _{h_{R} \in A} \operatorname{Ent}_{R}\left(h_{R}\right) \geqslant \operatorname{Ent}_{R_{n}}\left(\left\{h_{R_{n}} \in M\left(R_{n}, h_{\partial R_{n}}, \delta\right) \mid \tilde{h}_{R_{n}} \in A\right\}\right)-\theta_{A}(\delta)-\theta_{A, \delta}\left(\frac{1}{n}\right) .
$$

Note the analogy between (58) and inequality (49) from the proof of the variational principle. Indeed, we prove (58) in a similar manner to (49). We fix a sequence of asymptotic height function $h_{R}^{(k)} \in A$ that saturates the infimum; for concreteness, choose $h_{R}^{(k)}$ such that

$$
\operatorname{Ent}_{R}\left(h_{R}^{(k)}\right) \leqslant \inf _{h_{R} \in A} \operatorname{Ent}_{R}\left(h_{R}\right)+\frac{1}{k}
$$

Let $\theta^{(k)}(\delta)$ and $\theta_{\delta}^{(k)}\left(\frac{1}{n}\right)$ denote the error terms from the profile theorem for $h_{R}^{(k)}$. Choose a decreasing sequence $\delta_{k}$ such that:

(a) $\delta_{k} \leqslant \frac{1}{2} \delta_{k-1}$, so that $\delta_{k} \rightarrow 0$;

(b) $\delta_{k} \leqslant \frac{1}{2} \inf _{h_{R} \in A^{c}}\left\|h_{R}^{(k)}-h_{R}\right\|_{u}$; and

(c) $\theta^{(k)}\left(\delta_{k}\right) \leqslant \frac{1}{k}$.

Given $\delta>0$, choose $k$ such that $\delta \in\left(\delta_{k}, \delta_{k-1}\right]$. We claim that for large enough $n$ (depending on $A$ and $\delta$ ),

$$
B\left(R_{n}, h_{R}^{(k)}, \delta_{k}\right) \subseteq\left\{h_{R_{n}} \in M\left(R_{n}, h_{\partial R_{n}}, \delta\right) \mid \tilde{h}_{R_{n}} \in A\right\}
$$

Indeed, if $h_{R_{n}} \in B\left(R_{n}, h_{R}^{(k)}, \delta_{k}\right)$, then for $z \in \partial R_{n}$ and $x \in \partial R$,

$$
\begin{gathered}
\frac{1}{n}\left|h_{R_{n}}(z)-h_{\partial R_{n}}(z)\right| \leqslant\left|\frac{1}{n} h_{R_{n}}(z)-h_{R}^{(k)}\left(\frac{1}{n} z\right)\right|+\left|h_{R}^{(k)}\left(\frac{1}{n} z\right)-h_{\partial R}(x)\right| \\
+\left|h_{\partial R}(x)-\frac{1}{n} h_{\partial R_{n}}(z)\right| .
\end{gathered}
$$

By choosing $x$ close to $\frac{1}{n} z$ (which is possible, since $\frac{1}{n} R_{n}$ converges to $R$ ), the second error term tends to 0 . So does the third, because $\frac{1}{n} h_{\partial R_{n}}$ converges to $h_{\partial R}$ by hypothesis. Since $\delta_{k}<\delta$, this proves the inclusion $B\left(R_{n}, h_{R}^{(k)}, \delta_{k}\right) \subseteq M\left(R_{n}, h_{\partial R_{n}}, \delta\right)$. It remains to show 
that each $h_{R_{n}} \in B\left(R_{n}, h_{R}^{(k)}, \delta_{k}\right)$ satisfies $\tilde{h}_{R_{n}} \in A$. But by criterion (b) above, at every lattice point $z \in R_{n}$,

$$
\left|\frac{1}{n} h_{R_{n}}(z)-h_{R}^{(k)}\left(\frac{1}{n} z\right)\right| \leqslant \delta_{k}<\inf _{h_{R} \in A^{c}}\left\|h_{R}^{(k)}-h_{R}\right\| .
$$

Therefore $\left|\tilde{h}_{R_{n}}\left(\frac{1}{n} z\right)-h_{R}^{(k)}\left(\frac{1}{n} z\right)\right|<\inf _{h_{R} \in A^{c}}\left\|h_{R}^{(k)}-h_{R}\right\|$ for each $z \in R_{n}$, and since $\tilde{h}_{R_{n}}$ is extended from these points to $R$ by piecewise affine interpolation, the same inequality holds for all $x \in R$. In particular, this implies that $\tilde{h}_{R_{n}} \notin A^{c}$ and the inclusion (59) holds as claimed.

Using this inclusion together with the profile theorem for $h_{R}^{(k)}$, we derive the inequalities

$$
\begin{aligned}
\operatorname{Ent}_{R_{n}}( & \left.\left\{h_{R_{n}} \in M\left(R_{n}, h_{\partial R_{n}}, \delta\right) \mid \tilde{h}_{R_{n}} \in A\right\}\right) \\
& \leqslant \operatorname{Ent}_{R_{n}}\left(B\left(R_{n}, h_{R}^{(k)}, \delta_{k}\right)\right) \\
& \leqslant \operatorname{Ent}_{R}\left(h_{R}^{(k)}\right)+\theta^{(k)}\left(\delta_{k}\right)+\theta_{\delta_{k}}^{(k)}\left(\frac{1}{n}\right) .
\end{aligned}
$$

By choice of $h_{R}^{(k)}$, we have $\operatorname{Ent}_{R}\left(h_{R}^{(k)}\right) \leqslant \inf _{h_{R} \in A} \operatorname{Ent}_{R}\left(h_{R}\right)+\frac{1}{k}$. By criterion (c), we have $\theta^{(k)}\left(\delta_{k}\right) \leqslant \frac{1}{k}$. Since we chose $k$ such that $\delta \in\left(\delta_{k}, \delta_{k-1}\right]$, we have $k \rightarrow \infty$ and $\delta \rightarrow 0$, so $\frac{1}{k}=\theta_{A}(\delta)$. (The dependence on $A$ comes from the choice of $k_{R}^{(k)}$.) Finally, since $k$ is chosen based on $A$ and $\delta$, the last error term $\theta_{\delta_{k}}^{(k)}\left(\frac{1}{n}\right)=\theta_{A, \delta}\left(\frac{1}{n}\right)$. Putting all these observations together, we have proven (58):

$$
\inf _{h_{R} \in A} \operatorname{Ent}_{R}\left(h_{R}\right) \geqslant \operatorname{Ent}_{R_{n}}\left(\left\{h_{R_{n}} \in M\left(R_{n}, h_{\partial R_{n}}, \delta\right) \mid \tilde{h}_{R_{n}} \in A\right\}\right)-\theta_{A}(\delta)-\theta_{A, \delta}\left(\frac{1}{n}\right) .
$$

Now, we turn to the LDP upper bound (17), i.e.

$$
\varlimsup_{\delta \rightarrow 0} \varlimsup_{n \rightarrow \infty} \frac{1}{r_{\delta, n}} \log \mu_{\delta, n}(A) \leqslant-\inf _{h_{R} \in \bar{A}} I\left(h_{R}\right) .
$$

We observe that $\left(\mu_{\delta, n}\right)_{\delta, n}$ is exponentially tight, i.e. that for every $b \in(0, \infty)$, there exists $K_{b} \subset M(R)$ such that

$$
\varlimsup_{\delta \rightarrow 0} \varlimsup_{n \rightarrow \infty} \frac{1}{r_{\delta, n}} \log \mu_{\delta, n}\left(K_{b}^{c}\right) \leqslant-b .
$$

Indeed, we may take $K_{b}$ to be the closure of $M\left(h_{R}, h_{\partial R}, 1\right)$, independent of $b$. For $\delta<\frac{1}{3}$ and $n$ large enough that

$$
\max _{z \in \partial R_{n}}\left|\frac{1}{n} h_{\partial R_{n}}(z)-h_{\partial R}\left(\frac{1}{n} z\right)\right| \leqslant \frac{1}{3}
$$

any $h_{R_{n}} \in M\left(R_{n}, h_{\partial R}, \delta\right)$ satisfies $\tilde{h}_{R_{n}} \in M\left(h_{R}, h_{\partial R}, 1\right)$ by the triangle inequality, so $\mu_{\delta, n}\left(K_{b}^{c}\right)=0$. By the general theory of large deviations, exponential tightness implies that it is sufficient prove the upper bound (17) for compact sets $A \subset M(R)$. 
If $\inf _{h_{R} \in A} I\left(h_{R}\right)=\infty$, then every height function in $A$ differs from $h_{\partial R}$ at some point on the boundary. In fact by compactness, there exists $\delta_{0}$ such that for every $h_{R} \in A$, $\sup _{x \in \partial R}\left|h_{\partial R}(x)-h_{R}(x)\right| \geqslant \delta_{0}$. Clearly, as in the proof of exponential tightness above, this implies that $\left\{h_{R_{n}} \in M\left(R_{n}, h_{\partial R_{n}}, \delta\right) \mid \tilde{h}_{R_{n}} \in A\right\}$ is empty once $\delta$ is small enough and $n$ large enough. For all such $\delta, n$ we have $\mu_{\delta, n}(A)=0$ and (17) follows.

It remains to prove the upper bound (17) when $\inf _{h_{R} \in A} I\left(h_{R}\right)<\infty$ and $A$ is compact. Just like for the lower bound before, we reduce to proving the following inequality:

$$
\inf _{h_{R} \in A} \operatorname{Ent}_{R}\left(h_{R}\right) \leqslant \operatorname{Ent}_{R_{n}}\left(\left\{h_{R_{n}} \in M\left(R_{n}, h_{\partial R_{n}}, \delta\right) \mid \tilde{h}_{R_{n}} \in A\right\}\right)+\theta_{A}(\delta)+\theta_{A, \delta}\left(\frac{1}{n}\right) .
$$

We will closely follow the proof of (50) from the proof of Theorem 16 in Section 7 . Let $\varepsilon>0$, and choose $h_{R}^{(1)} \in A$ such that

$$
\operatorname{Ent}_{R}\left(h_{R}^{(1)}\right) \leqslant \inf _{h_{R} \in A} \operatorname{Ent}_{R}\left(h_{R}\right)+\varepsilon .
$$

As in (54), choose $h_{R}^{(2)}, \ldots, h_{R}^{(k)} \in A$ such that

$$
A \subset \bigcup_{i=1}^{k} B\left(R, h_{R}^{(i)}, \eta_{i}\right)
$$

where $\eta_{1}, \ldots, \eta_{k}$ are chosen so that for each $i$, the $\theta(\delta)$ term from the profile theorem for $h_{R}^{(i)}$ satisfies $\theta\left(\eta_{i}\right) \leqslant \varepsilon$. Exactly as in the proof of Theorem 16 (see (55)),

$$
\operatorname{Ent}_{R_{n}}\left(\left\{h_{R_{n}} \in M\left(R_{n}, h_{\partial R_{n}}, \delta\right) \mid \tilde{h}_{R_{n}} \in A\right\}\right) \geqslant-\frac{1}{\left|R_{n}\right|} \log \sum_{i=1}^{k}\left|B\left(R_{n}, h_{R}^{(i)}, \eta_{i}\right)\right| .
$$

From this we deduce the analogue of (56), namely

$$
\begin{aligned}
\operatorname{Ent}_{R_{n}}( & \left.\left\{h_{R_{n}} \in M\left(R_{n}, h_{\partial R_{n}}, \delta\right) \mid \tilde{h}_{R_{n}} \in A\right\}\right) \\
& \geqslant \operatorname{Ent}_{R}\left(h_{R}^{(1)}\right)+\theta_{A, \delta}\left(\frac{1}{n}\right)-5 \varepsilon \\
& \geqslant \inf _{h_{R} \in A} \operatorname{Ent}_{R}\left(h_{R}\right)+\theta_{A, \delta}\left(\frac{1}{n}\right)-6 \varepsilon .
\end{aligned}
$$

This completes the proof of (60) and of the large deviations principle.

\section{Acknowledgment}

The authors want to thank Tim Austin, Marek Biskup, Thomas Liggett, Igor Pak, Greta Panova for the fruitful discussions and helpful comments. We would also like to thank an anonymous referee for several helpful suggestions and corrections. This research has been partially supported by NSF grant DMS-1712632. 


\section{References}

[BCG16] Alexei Borodin, Ivan Corwin, and Vadim Gorin. Stochastic six-vertex model. Duke Math. J., 165(3):563-624, 2016.

[CEP96] Henry Cohn, Noam Elkies, and James Propp. Local statistics for random domino tilings of the Aztec diamond. Duke Math. J., 85(1):117-166, 1996.

[Cer06] Raphaël Cerf. The Wulff Crystal in Ising and Percolation Models, volume 1878 of Lecture Notes in Mathematics. Springer-Verlag, Berlin, 2006.

[CKP01] Henry Cohn, Richard Kenyon, and James Propp. A variational principle for domino tilings. J. Amer. Math. Soc., 14(2):297-346, 2001.

[CPT18] Nishant Chandgotia, Igor Pak, and Martin Tassy. Kirszbraun-type theorems for graphs. J. Comb. Theory Ser. B, 2018.

[CS16] Filippo Colomo and Andrea Sportiello. Arctic curves of the six-vertex model on generic domains: The tangent method. J. Stat. Phys., 164(6):1488-1523, 2016.

[Des98] Nicolas Destainville. Entropy and boundary conditions in random rhombus tilings. J. Phys. A, 31(29):6123-6139, 1998.

[DGI00] Jean-Dominique Deuschel, Giambattista Giacomin, and Dmitry Ioffe. Large deviations and concentration properties for $\nabla \phi$ interface models. Probab. Theory Relat. Fields, 117(1):49-111, 2000.

[DKS92] Roland Lvovich Dobrushin, Roman Koteckỳ, and Senya Shlosman. Wulff Construction, volume 104 of Translations of Mathematical Monographs. American Mathematical Society, Providence, RI, 1992. A global shape from local interaction, Translated from the Russian by the authors.

[DSS08] Daniela De Silva and Ovidiu Savin. Minimizers of convex functionals arising in random surfaces. Duke Math. J., 151, October 2008.

[Dur10] Rick Durrett. Probability: Theory and Examples. Cambridge Series in Statistical and Probabilistic Mathematics. Cambridge University Press, Cambridge, fourth edition, 2010.

[DZ09] Amir Dembo and Ofer Zeitouni. Large Deviations Techniques and Applications. Stochastic Modelling and Applied Probability. Springer Berlin Heidelberg, 2009.

[FO04] Tadahisa Funaki and Hirofumi Osada. Stochastic Analysis on Large Scale Interacting Systems. Advanced studies in pure mathematics. Mathematical Society of Japan, 2004.

[FS06] Patrik L. Ferrari and Herbert Spohn. Scaling limit for the space-time covariance of the stationary totally asymmetric simple exclusion process. Commun. Math. Phys., 265(1):1-44, 2006.

[JPS98] William Jockusch, James Propp, and Peter Shor. Random domino tilings and the arctic circle theorem. arXiv:math/9801068, 1998. 
[Kas63] Pieter W Kasteleyn. Dimer statistics and phase transitions. J. Math. Phys., 4:287-293, 1963.

[Kir34] Mojżesz D Kirszbraun. Über die zusammenziehende und Lipschitzsche Transformationen. Fundam. Math., 22(1):77-108, 1934.

[KK92] Claire Kenyon and Rick Kenyon. Tiling a polygon with rectangles. Proc. of 33rd Fundam. of Comput. Sci. (FOCS), pages 610-619, 1992.

[KMT18] Anndrew Krieger, Georg Menz, and Martin Tassy. A quenched variational principle for discrete random maps. arXiv:1710.11330, 2018. Preprint.

[KS99] Michael K.-H. Kiessling and Herbert Spohn. A note on the eigenvalue density of random matrices. Commun. Math. Phys., 199(3):683-695, January 1999.

[LP08] Lionel Levine and Yuval Peres. Strong spherical asymptotics for rotor-router aggregation and the divisible sandpile. Potential Anal., 30(1):1, 2008.

[LRS01] Michael Luby, Dana Randall, and Alistair Sinclair. Markov chain algorithms for planar lattice structures. SIAM J. Comput., 31(1):167-192, 2001.

[LS77] Benjamin F Logan and Larry A Shepp. A variational problem for random Young tableaux. Adv. in Math., 26(2):206-222, 1977.

[MT20] Georg Menz and Martin Tassy. A variational principle for a non-integrable model. Probab. Theory Relat. Fields, 177(3):747-822, 2020.

[PR07] Boris Pittel and Dan Romik. Limit shapes for random square Young tableaux. Adv. in Appl. Math., 38(2):164-209, 2007.

[RAS15] Firas Rassoul-Agha and Timo Seppäläinen. A Course on Large Deviations with an Introduction to Gibbs Measures. American Mathematical Society, May 2015.

[RS18] Nicolai Reshetikhin and Ananth Sridhar. Limit shapes of the stochastic six vertex model. Commun. Math. Phys., 363(3):741-765, 2018.

[Sch14] Jean Van Schaftingen. Approximation in Sobolev spaces by piecewise affine interpolation. J. Math. Anal. Appl., 420(1):40-47, 2014.

[She01] Scott Sheffield. Ribbon tilings and multidimensional height functions. Trans. Am. Math. Soc., 354, August 2001.

[She05] Scott Sheffield. Random Surfaces, volume 304 of Astérisque. Société mathématique de France, 2005.

[SS95] Lynn Arthur Steen and J Arthur Seebach. Counterexamples in Topology. Dover books on mathematics. Dover Publications, 1995.

[Thu90] William P. Thurston. Conway's tiling groups. Am. Math. Monthly, 97(8):757756, September 1990.

[VK77] Anatolii Moiseevich Veršik and Sergei V. Kerov. Asymptotic behavior of the Plancherel measure of the symmetric group and the limit form of Young tableaux. Dokl. Akad. Nauk SSSR, 233(6):1024-1027, 1977. 
[Wig59] Eugene Paul Wigner. Statistical properties of real symmetric matrices with many dimensions. In 4th Can. Math. Congress (Banff 1957), pages 174-184. Univ. Toronto Press, 1959.

[Wil04] David Bruce Wilson. Mixing times of lozenge tiling and card shuffling Markov chains. Ann. Appl. Probab., 14(1):274-325, February 2004. 